THE RANK AND MODEL SPECIFICATION OF DEMAND SYSTEMS: AN EMPIRICAL ANALYSIS USING UNITED STATES MICRODATA

Christopher J. Nicol

Department of Economics

University of Regina

Regina, Saskatchewan

Canada S4S 0A2

econ@uregina.ca

JULY 1998 


\title{
The Rank and Model Specification of Demand Systems: An Empirical Analysis Using
} United States Microdata

\author{
by \\ Christopher J. Nicol \\ Department of Economics \\ University of Regina \\ Regina, Saskatchewan, S4S 0A2 \\ CANADA \\ Internet: Chris.Nicol@URegina.CA; \\ http://athena.cas.uregina.ca
}

July 21,1998

The support of the Social Sciences and Humanities Research Council of Canada is gratefully acknowledged. I thank James Farley for outstanding research assistance, and participants at the presentation of an earlier version of this paper at the Annual Meetings of the Canadian Economics Association, Ottawa, Ontario, June, 1998 for their helpful comments. Any errors are my responsibility. 


\begin{abstract}
A rank three demand system incorporating labour force participation, non-separability of demands from excluded goods and non-exact aggregation in income and household characteristics is estimated using United States Consumer Expenditure Survey microdata. A unique price data set is used in conjunction with these microdata, which permits the analysis of systematic errors in price variables, and their effects on hypothesis tests and estimated elasticities. Various alternative separability assumptions are also incorporated in the estimated models, in order to analyse the implications of these effects as well.

It is found that errors in price variables bias test results for the rank three hypothesis in particular towards rejection. Other test results are also affected in the way, but to a lesser degree. It is also found that estimating smaller systems of demand equations, even when conditioning on excluded goods yields significantly different test results and estimated elasticities. There is also evidence that the effects of errors in price variables bias tests in one direction, and in the opposite direction under different separability assumptions.

Another important conclusion is that model specification can vary significantly for households of different family sizes and housing tenure status. This indicates that caution should be exercises when presented with demand estimation results based on samples with households with heterogeneous characteristics.
\end{abstract}

\title{
Keywords:
}

errors in variables; labour supply; microdata; rank three demands; separability. 


\section{Introduction}

Recent research in applied demand analysis has focused on a variety of model specification issues. For example: whether demand systems are rank two or three (Fry and Pashardes, 1992 and Banks, Blundell and Lewbel, 1997); the separability of commodity demands from labour supply (Browning and Meghir, 1991 and Kaiser, 1993); and the role of household characteristics variables in demand models (Blundell, Pashardes and Weber, 1993 and Dickens, Fry and Pashardes, 1993).

These studies have indicated that each of the above features are important determinants of demand. In these applications, however, not all of these modelling considerations are controlled for simultaneously. That is, one or more of these features are not explicitly modelled in all of the applications mentioned. Since the various modelling aspects have been found to be important determinants of demand, applications which focus on one or more but ignore others gives cause for concern. This was highlighted in Dagenais (1994), where the effects of multiple model specification errors were investigated. It was found that, when

more than one model specification error was present, correcting for only one induced larger biases and inconsistencies than if one did not control for any of them.

It is also well-known that errors in variables can give rise to serious distortions in estimation results when these errors are ignored (see Cragg, 1994, for a recent survey article on this issue). In the context of demand system estimation using microdata, it can be said almost unequivocally that research routinely ignores the presence of gross errors in the price variables used in estimation. For example, such research using United Kingdom (UK) data identifies price parameters solely through the variation in prices over time, though it is known that regional variation in prices exists in the UK. Furthermore, research employing the Canadian microdata has used regional and temporal price variation to identify price parameters, yet there still exist errors in price variables associated with using regional as opposed to city-level price indices.

Of course, there are very good reasons for ignoring the errors in prices indicated above. To begin with, there is often no more information readily available on prices than that which is used. In addition, even when information is available on the variability in prices at a regional level, it would be rather complicated to include this in an estimation procedure which is already typically rather difficult.

It can thus be seen as an important task to develop a detailed study which focuses on all of the determinants of demand which have been found to be important (as described above), and on the indicated errors in variables problem, in a comprehensive estimation strategy. Such a study could shed light on the possible effects the interactions of these features might have. To facilitate this, one would therefore require 
a very rich data set, covering a geographic region where prices vary considerably. In addition, it would be helpful if variations in prices were well documented. Of course, the United States is a country which has a long series of microdata sets. These data have been used to a limited extent in demand analysis, partly owing to the problem that inter-regional price comparisons are not published by government agencies. There are consumer price data for a wide range of cities, states and regions, but these compare prices across time for specific cities or region; not across cities at a point in time. In this paper, price data are constructed based on the US consumer price index (CPI) and American Chamber of Commerce Research Association (ACCRA) data. These latter price data comprise indices for a range of products across US cities over time, and are comparable across those cities at the points in time covered by the data.

US Consumer Expenditure Survey (CES) microdata are used to analyse the model specification issues discussed earlier, and also the possible influence on estimation results of errors in price variables. Given the unique nature of the price data used, it is possible to analyse the effects on hypothesis tests and estimated elasticities of induced errors in price variables, where the nature of the errors are similar to the kinds encountered by researchers who have used the UK and Canadian data. The importance of labour force participation effects, the rank of the demand system and the effects of separability of the goods in the demand model from other goods can also be analysed.

It is found that estimated coefficients, test results and estimated elasticities are significantly different when estimated via a separable demand sub-system, as opposed to estimating a larger system where all goods are included directly. This is the case even when the separable sub-system contains features which control for the omission of goods not included directly. It is also found that a rank two demand specification is adequate for most of the household groups considered. This is in contrast with the result of Banks, Blundell and Lewbel (1997), although their study focused on only one specific household type. What the results in this paper suggest is that the rank result is highly dependent on the type of household group considered, so care should be exercised when modelling this aspect. Furthermore, it is the case that the rank results appears to be significantly affected by errors in price variables. In the presence of these errors, rank appears to be three; but when these errors are controlled for, rank appears to be two for all but one of the household groups considered.

With respect to estimated elasticities, these are affected significantly both by errors in price variables and the size of the system estimated. In terms of other model specification matters, the results in this paper bear out earlier findings regarding the importance of labour force participation effects as determinants of 
demand, the effects of age of head of household and several other variables typically believed to affect demand patterns.

The remainder of the paper is structured as follows. In Section 2, model specification is discussed. Some relevant empirical literature is also discussed, which gives direction to the initial model specification. The data used are discussed in Section 3. Section 4 gives details of the estimated models, hypothesis tests conducted, and provides comparisons of the estimated elasticities under the various estimation conditions. Section 5 summarises and concludes.

\section{Model Specification}

Suppose "goods" over which consumers make decisions can be partitioned into four types. Goods of direct interest, denoted $q$, and their prices, $p$; labour force variables, $\ell$; other conditioning goods, $c$; and demographic or household characteristics variables, $z$. If preferences can be represented by the utility function, $U[q, \ell, c, z]$, the conditional cost function is defined as $c[p, \ell, c, z, u]=\min _{q}[p \cdot q \mid U(q, \ell, c, z)=u]$. The properties of these functions are discussed in Pollak (1969) and Browning (1983). All variables in $q, \ell, g$ and $z$ can be vectors. The conditional, compensated demand functions for $q$ are the derivatives of this cost function with respect to $p$ and can be denoted $q_{i}=f_{i}[p, \ell, c, z, y]$, where $y$ is total expenditure $(y)$ on the $n$ goods, $q=\left[q_{1}, \ldots, q_{n}\right]^{T}$. Note that $n$ does not include goods which comprise expenditures in $c$.

To characterise a demand system which is of rank three (where the rank of the system refers to the dimension of the space spanned by the system's Engel functions - Lewbel, 1990), a quadratic generalisation of the Almost Ideal (AI) demand system of Deaton and Muellbauer (1980) is used. This quadratic AI (QAI) system nests the AI model, and is less restrictive than the quadratic expenditure system (QES) used by Howe, Pollak and Wales (1978), the most general form of which was presented in van Daal and Mierkes (1989). The most general case of the QAI system was developed in Lewbel (1991), then used in empirical applications by Banks, Blundell and Lewbel $(1992,1997)$ and Fry and Pashardes (1992).

Using notation similar to that in Fry and Pashardes (1992), the cost function for the QAI model is represented by

$$
\ln c[p, \ell, c, z, u]=a(p, \ell, c, z)+\frac{b(p, \ell, c, z)}{[f(u)-g(p, \ell, c, z)]}
$$

which can be re-arranged to yield the indirect utility function

$$
\ln V[p, y ; \ell, c, z]=f^{-1}\left\{\frac{b(p, \ell, c, z)}{[\ln y-a(p, \ell, c, z)]}+g(p, \ell, c, z)\right\}
$$


Fry and Pashardes (1992) show that this indirect utility function yields the budget-share system

$$
w_{i}=a_{i}^{\prime}(p, \ell, c, z)+\frac{b_{i}^{\prime}(p, \ell, c, z)}{b(p, \ell, c, z)}[\ln (y-a[p])]+\frac{g_{i}^{\prime}(p, \ell, c, z)}{g(p, \ell, c, z)}[\ln (y-a[p])]^{2}
$$

where $a_{i}^{\prime}(p, \ell, c, z), b_{i}^{\prime}(p, \ell, c, z)$ and $g_{i}^{\prime}(p, \ell, c, z)$ are derivatives of the respective functions $a(p, \ell, c, z), b(p, \ell, c, z)$ and $g(p, \ell, c, z)$ with respect to $\ln p_{i}$.

The way in which the three functions, $a[\cdot], b[\cdot]$ and $g[\cdot]$ are parametrised permits the demand system to be conditioned on labour force participation variables, household characteristics variables, and other expenditures not included directly in the demand system. Furthermore, the presence of the function, $g[\cdot]$, is the feature of the model which delivers Engel functions which are quadratic in functions of total expenditures. That is, Engel functions which span a three-dimensional sub-space.

The functional forms adopted for $a[\cdot], b[\cdot]$ and $g[\cdot]$ are taken from Fry and Pashardes (1992), as follows

$$
a(p, \ell, c, z)=\alpha_{0}+\sum_{i} \alpha_{i}(\ell, c, z) \ln p_{i}+\frac{1}{2} \sum_{i} \sum_{j} \gamma_{i j} \ln p_{i} \ln p_{j}
$$

which is the familiar functional form for $a[\cdot]$ from the AI system, but here permitted to depend on $\ell, c$ and $z$,

$$
b(p, \ell, c, z)=\beta_{0} \prod_{i} p_{i}^{\beta_{i}(\ell, c, z)}
$$

again taking the form from the AI system, but incorporating the effects of $\ell, c$ and $z$, and

$$
g(p, \ell, c, z)=b(p, \ell, c, z) \cdot \lambda(p, \ell, c, z)
$$

where

$$
\lambda(p, \ell, c, z)=\lambda_{0}+\sum_{i} \lambda_{i}(\ell, c, z) \ln p_{i}
$$

The specifications of (6) and (7) are convenient, in that these yield budget-shares without across-equation restrictions on quadratic expenditure effects, and the inclusion of $\lambda_{0}$ removes effects of price normalisation on the other parameters of the model (Fry and Pashardes, 1992, p. 5).

The following linearisations of the functions $\alpha_{i}(\ell, c, z), \beta_{i}(\ell, c, z)$ and $\lambda_{i}(\ell, c, z)$ complete the specification

$$
\begin{gathered}
\alpha_{i}(\ell, c, z)=\sum_{i} \sum_{k}\left[\alpha_{i k} v_{k}\right] \ln p_{i} \\
\beta_{i}(\ell, c, z)=\sum_{i} \sum_{k}\left[\beta_{i 0}+\beta_{i k} v_{k}\right] \ln p_{i}
\end{gathered}
$$




$$
\lambda(p, \ell, g, z)=\lambda_{0}+\sum_{i} \lambda_{i} \ln p_{i}
$$

where the vector $\nu=\left[\nu_{1}, \ldots, \nu_{K}\right]^{T}$ is used to represent $\ell, c$ and $z$, for notational convenience. The influences of $\ell, c$ and $z$ are therefore confined to $\alpha_{i}(\ell, c, z)$ and $\beta_{i}(\ell, c, z)$.

The model, (3), encompasses a variety of effects, all of which have been found to be important determinants of demand on their own. Whether these effects should enter the model simultaneously, or whether some are capturing more than one influence can therefore be explored, given the above specification.

Integrability conditions which the parameters of the model must satisfy, in order to be consistent with the indirect utility function specified above, are: $\sum_{i} \alpha_{i 1}=1, \sum_{i} \alpha_{i k}=0, k=2, \ldots K, \sum_{i} \gamma_{i j}=0, \sum_{i} \beta_{i k}=0$ all $k$, and $\sum_{i} \lambda_{i}=0$, to satisfy adding up; $\sum_{j} \gamma_{i j}=0$ to satisfy homogeneity; and $\gamma_{i j}=\gamma_{j i}, \forall i \neq j$, to satisfy symmetry of substitution effects. Of course, a negativity condition is also required of the Slutsky matrix for the model, but this cannot be satisfied globally for this class of system. In the empirical application which follows, the integrability conditions are imposed (apart from negativity), since earlier work indicated that homogeneity and symmetry restrictions could not be rejected for these data (Nicol, 1995).

Given the above parameterisations for (4)-(10), the following budget-share system can be obtained:

$$
\begin{aligned}
w_{i}= & \sum_{k} \alpha_{i k} \nu_{k}+\sum_{j} \gamma_{i j} \ln p_{j}+\left[\beta_{i 0}+\sum_{k} \beta_{i k} \nu_{k}\right][\ln (y)-a(p, \nu)]+ \\
& \left\{\lambda_{i}+\left[\beta_{i 0}+\sum_{k} \beta_{i k} \nu_{k}\right]\left[\lambda_{0}+\sum_{i} \lambda_{i} \ln p_{i}\right]\right\}[\ln (y)-a(p, \nu)]^{2}+\mu_{i}
\end{aligned}
$$

The random term, $\mu_{i}$, denotes a stochastic disturbance such that $\left[\mu_{1}, \ldots, \mu_{n}\right]^{T} \sim \mathrm{N}(0, \Omega)$. The covariance matrix of $\mu$ is singular, so only $n-1$ equations of the system need to be estimated, the parameters of the $n$th being recoverable by the adding-up conditions indicated above. Empirical considerations relating to this stochastic specification will be discussed in Section 4.

Many hypotheses can be tested using (11), via zero restrictions on the parameters, $\alpha_{i k}, \beta_{i k}$ and $\lambda_{i}$. Restricting the model to a rank two system requires that $\lambda_{0}=\lambda_{i}=0$, for all $i$. Separability of commodity demands from labour supply requires that the $\alpha_{i k}$ and $\beta_{i k}$ parameters associated with labour force participation be zero, and so on. Also, the interaction of household characteristics variables (in the $\nu$ vector) with $[\ln (y)-a(p, \nu)]$ yields a non-exactly aggregable demand system. To control for the most important household characteristics effects (as evidenced in the work of Barnes and Gillingham, 1984, and Nicol, 1989), six different household group sub-samples, based on family size and housing tenure status, were employed. Other variables, such as labour force participation effects, were included directly via the vector, $\nu$. Details of the overall approach will be explained further in Section 4. 
Elasticity estimates were also calculated, given the following parameterisations for income elasticities $\left(\eta_{i}\right)$, compensated price $\left(\epsilon_{i j}^{*}\right)$ and uncompensated price $\left(\epsilon_{i j}\right)$ elasticities respectively

$$
\begin{aligned}
\eta_{i}= & \left\{\beta_{0}+\sum_{k} \beta_{i k} \nu_{k}+2\left[\lambda_{i}+\left(\beta_{0}+\sum_{k} \beta_{i k} \nu_{k}\right) \cdot \lambda_{0}\right]\left(\ln (y)-\alpha_{0}\right)\right\} / w_{i}+1 \\
\epsilon_{i j}^{*}= & {\left[1 / w_{i}\right]\left[\gamma_{i j}+\left(\beta_{0}+\sum_{k} \beta_{i k} \nu_{k}\right) \lambda_{j}\left(\ln (y)-\alpha_{0}\right)^{2}+w_{i}\left(\eta_{i}-1\right)\left(w_{j}-\sum_{k} \alpha_{j k} \nu_{k}\right)\right]+} \\
& w_{j}-\delta_{i j} \\
\epsilon_{i j}= & \epsilon_{i j}^{*}-w_{j} \eta_{i}
\end{aligned}
$$

where $\delta_{i j}=1$ if $i=j$ and 0 otherwise. It should be noted that these elasticity formulae take into account evaluation at normalised prices, $p=[1, \ldots, 1]^{T}$, which is a zero vector when logs are taken, and that evaluation further requires a value for the vector $\nu$. The vector of sample means was used for this purpose. Also, $\ln y=1$ at the mean of total expenditures, $\bar{y}$, by appropriate normalisation of $y$.

\section{Data}

The expenditure data for this study are drawn from the 1980-81, 1982-83, and the annual, 1984-1992 Interview Survey Public-Use Tapes of the CES for the United States. The procedure for collecting data from households in the CES samples was as follows. Each sample was split into three monthly rotation groups. Households in a rotation group were then interviewed in the "same" month of each quarter for five consecutive quarters, reporting their spending patterns for the preceding quarter. This yields one rotation group reporting their quarterly expenditures every month. Households can therefore be matched to monthly price data, since it is known when a household reports, and for which quarter. In contrast, in the Canadian case, households report expenditures for the a year, so this limits the price information which can be used when working with the FAMEX.

At any time, there are approximately 5000 households in the CES Interview Surveys. Pooling data from the eleven indicated surveys permits construction of large samples of different households groups. The characteristics of households within any one group are chosen to be as similar as possible. Key characteristics variables are used to split samples into sub-groups having relatively similar characteristics within the group, but having a range of characteristics across groups. The choice of such "stratifying variables" is based on experience in applied demand research where such variables are associated with statistically significant differences in model parameters across groups.

Family size and housing tenure have been found to be of such importance in other demand studies (for 
example, Barnes and Gillingham, 1984, and Nicol, 1989), as to merit stratifying households into groups based on these variables. Household types were therefore classified according to three different family sizes: married couples without children; married couples with one child; and married couples with two children. Also, two types of housing tenure status were used to further classify the households: renter households; home-owners with mortgages; and home owners without mortgages. While it is possible to construct samples with larger family sizes and home-owner families without mortgages, such samples are significantly smaller, and are not the focus of this paper. For all six household types, only those with age of head 18-65 and no self-employed members were included in the sub-samples. Each of the respective household sizes and housing tenure statuses are distinguished in what follows by the identifiers: MOR0MOR2 for home-owners; and REN0-REN2 for renters, the ending digit indicating the number of children in the household.

The next step in creating the data sets to be used is the selection of expenditure categories of interest. This choice is governed by a number of considerations. The CES surveys disaggregate expenditures into various categories, so these categories are the minimum level of disaggregation one can work with. However, the kinds of categories in the surveys are similar to those used in surveys in other countries (such as Canada and the UK). Consequently, the expenditure categories used in this study can be chosen to be as close as possible to those in other studies.

The choice of expenditure categories is also dictated by the availability of price data with which to match expenditures. In addition, the more categories included in a demand system, the greater the number of parameters which have to be estimated. Such estimation is difficult in a nonlinear setting. Large systems can be made smaller by aggregating goods. However, inappropriate aggregation of expenditures can lead to misleading inferences (Nicol, 1991, provides some evidence on this in a homogeneity and symmetry testing context). Consequently, a small, disaggregated demand system is preferable from this perspective. There is then the danger, however, of excluding non-separable goods from such a system. Even including the effects of "other goods" in the form of a conditional demand system could lead to misleading inferences, for a variety of reasons. For example, the way in which these other goods influence demand behaviour could be intimately related to their distribution by expenditure type within the other goods category. This distributional information is completely obscured when employing an aggregate other good as an explanatory variable. There has been no research on the kinds of effects which might arise, so there is no way of knowing a priori whether one need be concerned over the nature and magnitude of these possible 
effects.

Given the above considerations, a maximum of nine expenditure categories were chosen for inclusion in the largest possible demand system to be estimated. These were: food (F), alcoholic beverages (A), clothing $(\mathrm{C})$, gasoline and fuel $(\mathrm{G})$, other automobile expenditures $(\mathrm{O})$, public transportation (P), household operation $(\mathrm{H})$, personal care spending $(\mathrm{E})$ and health care spending $(\mathrm{S})$. All other expenditures were dealt with as an aggregate conditioning good (CG). The categories included in CG were: shelter; household furnishings; auto purchase; tobacco, entertainment; education and other expenditures. Complete details of these expenditure categories are contained in the Interview Survey Public-Use Tape Documentation.

A demand system with nine equations and the complexity of the QAI model presented above, contains many parameters. For the specification considered here, there are 158 parameters in the nine equation system. The enhanced degree of variability in prices of the data used in this study permits the empirical identification of all these parameters for the six household sub-groups, MOR0-MOR2 and REN0-REN2. This is not routinely the case when using microdata for other countries, where only smaller systems can be estimated. It is, however, of interest to analyse the impact of estimating smaller systems, even when a good such as CG in included, for the reasons alluded to earlier. To this end, not only was the nine equation system (FACGOPHES) estimated, but so also were four other systems. These were three, three equation systems, which included: F, A and C (the FAC system); G, O and P (the GOP system); and H, E and $\mathrm{S}$ (the HES system). In addition, a six-equation system including F, A, C, G, O and P (the FACGOP system) was estimated. For these reduced systems, CG not only includes those expenditures indicated above for the FACGOPHES system, but also includes those expenditures for the nine equation system not included as equations for the reduced system. For example, for the FAC system, CG would include expenditures on G, O, P, H, E and S, as well as those expenditures included in CG for the FACGOPHES system.

Estimating the range of five systems indicated above means that test results and estimated elasticities can be compared for individual systems or goods across estimation situations. In particular, test results for rank three demands can be compared for the FAC versus FACGOPHES system and so on, and estimated elasticities based on these two systems can be compared for F, A and C.

As the model specification discussion in Section 2 indicated, labour force participation effects were to be included in the demand equations. The CES data contain information on the labour force participation status of adult household members. Consequently, these effects were introduced as labour force partic- 
ipation dummy variables. One variable was included for each of the adult male and female household members. In addition, these dummy variables were interacted with other variables on the right hand side of the estimating equations, as indicated in equation (11).

Other household characteristics effects included in the vector $\nu$ were age of the head of the household, a tobacco consumption dummy variable and a vehicle ownership dummy variable. These variables have been found to be important determinants of demand in other studies. (See, for example, Banks, Blundell and Lewbel, 1997).

Given the six samples of households from the CES, the next step is to match these households to the price vectors which they faced for the goods included in the demand system. There are several other variables in the CES which influence how this matching can be done. These are the variables giving household location information. The relevant variables are: region of residence; city population size in region of residence; and state of residence. There are four regions of residence: Northeast; Midwest; South; and West. Also, there are five population sizes. These change slightly over the eleven CES data sets used. Also, from 1980-85, the following states were not covered by the CES: Delaware, Idaho, Nevada, New Hampshire, North Dakota, Oklahoma, South Dakota, Vermont and Wyoming. From 1986-92, the following states were not covered: Montana, Nevada, North Dakota, Rhode Island, South Dakota, Vermont and Wyoming.

For certain households, some or all of the above variables were suppressed by the Bureau of Labor Statistics, in the interests of confidentiality of the respondents. However, using these three variables when not suppressed, it was possible to identify the city in which a household lived. In surveys prior to 1985, one could determine the households' cities of residence for twenty-seven US cities by cross-tabulating on the three variables - region, city population size and state. These cities are listed in Table A1 of the Appendix, along with a series of price indices, which will be discussed below. From the 1986 survey and onwards, however, the population size variables were suppressed for all households in the western states. These states were: Arizona, California, Colorado, Oregon, Utah and Washington. In that case, it was still possible to identify city of residence of households living in the twenty cities in the Northeast, Midwest and South listed in Table A1. Also, state of residence could be identified for households in West states, and their city of residence inferred as being one of a small sub-set of cities within each of these states.

The change in reporting of household location variables from 1986 and thereafter had implications for the way that price data could be constructed. For households in cities in the Northeast, Midwest and 
South, city of residence could be determined for the whole period, 1980-1992. These households could therefore be assigned city prices. On the other hand, for households in the West from 1986-92, only state of residence could be determined exactly, so these households had to be matched to state level prices. This resulted in the introduction of errors in price variables for households in the West for such observations. These types of errors are commonly seen in other data sets (for example, Canadian FAMEX-based data sets, and United Kingdom FES-based data sets), as was discussed earlier. Consequently, the effects of these errors in price variables can be assessed by comparing results based on different sub-samples for the non-West regions after 1986, and for all regions for data from 1980-85. In addition to distinguishing amongst the six household types, MOR0-MOR2 and REN0-REN2, estimation was also carried out: 11sing four-region data from 1980-1992; three-region data from 1980-1992 (excluding households from the West); and four-region data for households from all twenty-seven cities from 1980-1985. Summary statistics of the expenditures in these eighteen data sets, and their sample sizes are given in Table A2 of the Appendix.

The CES also indicate the month in which a household is interviewed. In principle, a household could thus be assigned a price vector reflecting when they made their expenditures. With twelve monthly price observations per year in thirteen years for up to twenty-seven cities, price data would contain a lot of information. Price effects could then be determined precisely. However, in the case of the CES, although detailed city-level CPI data are available and these can be matched to households at the city level, CPI data do not reflect how prices change over time for the specific cities. That is, city-level CPI's do not indicate whether prices in New York are higher than in Los Angeles for the same good, such as F. Fortunately, an extensive database of price indices for six categories each year, and for many cities, is available. These indices are published by the American Chamber of Commerce Researchers Association (ACCRA). The expenditure categories covered are: grocery items, housing, utilities, transportation, health care and miscellaneous goods and services. This is a smaller number of categories than are included in the FACGOPHES system. The ACCRA categories are therefore assigned to the FACGOPHES categories as closely as possible, given the expenditures included in the respective categories. These assignments are given in the Appendix Notes.

A representative sample of the ACCRA data (which also lists the twenty-seven cities covered in this study) is presented in Table A1 of the Appendix. These data, and others like them, were used in conjunction with city level CPI data to construct inter-city price indices for the cities in Table A1. Table A3 of the Appendix presents the initial, published CPI data for Philadelphia. These data were then converted, using 
appropriate series of ACCRA data, to series such as that in Table A4 of the Appendix. The Table A4 data are monthly inter-city price indices covering all categories of expenditure in the FACGOPHES system. Similar monthly series were created for all twenty-seven cities from 1980-85, and for the reduced set of twenty cities from 1986-92.

As well as city-level data, ACCRA data were also used in conjunction with appropriate CPI data for different city sizes in the West to obtain state-level data from 1986-92 for these states. A representative sample of these is given for Utah, in Table A5 of the Appendix.

City and state level price data were then matched to appropriate households in the various samples. Further details of the price construction procedure, matching to households, and extraction of actual households from the complete CES are available on request.

\section{Estimation and Results}

\subsection{Exogeneity of Explanatory Variables}

It is becoming increasingly common in applied demand studies using microdata for estimation to be conducted using an instrumental variables procedure. This is because of concern over purchase infrequency in some bodies of data, which calls into question the exogeneity of total expenditures $(y)$. The inclusion of labour force participation variables also lends strong support to this approach, owing to the possible endogeneity of these variables. Also, in the current study, the conditioning of demands on: expenditures outside the demand system; tobacco consumption; and vehicle ownership, raise the endogeneity issue too, since such decisions are not independent of allocating expenditures to goods in any of the five demand systems to be estimated.

In earlier work (Nicol, 1995), it was determined that instrumental variables estimation was appropriate for the data used here. The results which follow are thus based on this approach, with the variables included in the matrix of instruments, $W$, being defined and their summary statistics given in Table A6 of the Appendix. Identifiability of the parameters of the model was also established on the basis of these instruments, in Nicol (1995). Estimation was conducted using the GMM procedures in TSP, Version 4.3,

allowing for heteroskedasticity of unknown form in the computation of the variance-covariance matrices of the systems. 


\subsection{Model Specification Tests}

The model specification tests to be conducted involved various zero restrictions. The restriction that $\lambda=\left[\lambda_{0}, \lambda_{1}, \ldots, \lambda_{n}\right]^{T}=0$, reduces the system to one of rank two demands. The importance of age of head, labour force participation, conditioning goods, vehicle ownership and tobacco consumption as determinants of demand can also be analysed by tests of exclusion restrictions for the respective variables.

Tables 1-10 summarise the results of tests of the various hypotheses for the six household types, MOR0MOR2 and REN0-REN2. Tables 1 and 2 contain the results for the FACGOPHES system. Tables 3 and 4 contain corresponding results for the FACGOP system, Tables 5 and 6 for the FAC system, Tables 7 and 8 for the GOP system and Tables 9 and 10 for the HES system. Each of the tables contains results of tests based on estimation using the three different variations of data by regional dimension: four-region data from 1980-1992; four-region data from 1980-1985; and three-region data from 1980-1992. All of the test statistics in Tables 1-10 are based on the Wald principle of inference, calculated as the quadratic form of the vector of coefficients to be excluded under the null, with the inverse of the variance-covariance sub-matrix of those parameters. The test statistics are distributed as $\chi^{2}$ under the null, with degrees of freedom given by the number of exclusion restrictions. All further details are contained in the Notes to Tables 1-10.

In Tables 1 and 2, test results based on the four-region data from 1980-1992 indicate that all null hypotheses except one would be rejected at a significance level of 0.001 , which is a conservative $\alpha$ level given the sample sizes involved. (Table A2 of the Appendix summarises these sample sizes). If one considers instead corresponding results based on four-region data from 1980-1985, or on three-region data from 1980-1992 (which do not contain the errors in price variables discussed earlier, but which are present in the four-region data from 1980-1992), there is no strong evidence in favour of the system having a rank of three (with only one rejection out of twelve tests).

With respect to other model specification considerations, labour force participation effects, age of head, vehicle ownership and the conditioning goods excluded from the system also appear to be important determinants of demand for the MOR0-MOR2 households, using the 1980-1985 and three-region data. In contrast for REN0-REN2 households, there is virtually no evidence against any of these null hypotheses based on the data which are relatively free from errors in price variables.

Tables 3 and 4 contain the corresponding test results for the FACGOP system. The only difference in implementation between these results and those in Tables 1 and 2 is the coverage of goods in the system. 
In FACGOP, HES expenditures are included in CG (or $g$ ), whereas in FACGOPHES, HES expenditures are included in the system directly. Any variation in corresponding test outcomes across Tables $1-4$ is thus due to the restrictions in FACGOP relative to FACGOPHES, and those have to do with aggregation of goods and the nature of separability amongst the goods which is assumed.

Comparing the MOR0-MOR2 results in Table 3 versus those in Table 1 for four-region data from 198092 , there are twelve rejections in the former and eighteen (the maximum possible number) in the latter. There are also noticeably fewer rejections in Table 3 for four-region data from 1980-85, and three-region data from 1980-92 than in Table 1. Evidence in support of rank three demands is also stronger in the FACGOP system, when using relatively price error-free data. However, support for excluding age of head, labour force participation, vehicle ownership and tobacco consumption effects are weaker in Table 3 for the same data. As one might expect if aggregation and separability considerations are important, the evidence against excluding CG is almost equally as strong in Table 3 as in Table 1. The aggregation effects on tests thus appear to bias some results towards rejection, and others towards non-rejection for MOR0-MOR2 households.

A comparison of rejections for REN0-REN2 households in Table 4 with those in Table 2 also indicates noticeably fewer rejections for the latter than the former when considering 1980-92 four-region data results. Rank three, labour force participation and CG effects do not appear to be important determinants of demand for renters, based on the FACGOP system. On the other hand, more rejections are observed when basing comparisons on the relatively price error-free data. It therefore appears that errors in price variables and goods included directly in the demand system have major effects on test results, even when the demand system is conditioned on the excluded goods. With respect to the rank of the demand system, both kinds of specification error appear to influence results in the direction of rejection (even though tests in Table 4 do not reject the null, the upper-tail prob. values reported are lower for these tests than they are for corresponding tests in Table 2).

The final sets of test results deal with the much smaller FAC, GOP and HES systems. Total rejections across all three systems for 1980-1992 four-region data are sixteen (out of a total of one-hundred and eight tests). Corresponding rejections for 1980-1985 four-region data and 1980-1992 three-region data are ten in both cases. There is no systematic pattern to these rejections, except that rank three rejections are concentrated in the 1980-1992 four-region data results. This can again be interpreted as evidence that the errors in price variables for these data are affecting test outcomes. It also seems likely that estimation of 
these reduced systems yields results which are less precise than those obtained via estimation of the larger FACGOP and FACGOPHES systems.

Evidence for the importance of labour force participation as a determinant of demand is very weak for MOR0-MOR2 households, based on these tests, which appears to give a misleading impression, when compared to the more general FACGOPHES results. This is also the case for the tests for the exclusion of age of head, vehicle ownership, tobacco consumption and CG for the same households.

The foregoing test results indicate the importance for their outcomes of errors in price variables, and illustrate the impact of variation in the aggregation/separability assumptions made with respect to goods included in the demand system. These effects appear to bias rank three hypothesis tests towards rejection when errors in price variables are a specification error problem, and in the direction of non-rejection for the various other hypothesis tests, in terms of the aggregation/separability assumption being made. In the following sub-section, estimated elasticities are compared across the various régimes, to determine how they are affected by these sources of specification error.

\subsection{Estimated Elasticities}

Uncompensated price and income elasticities were computed based on the parameter estimates of each of the five systems, six household types and three regional groupings, given the formulae in equations (12)-(14) of Section 2. Again, the initial focus is on estimates based on the FACGOPHES system. These estimates are given in Tables 11-19. Tables 11-13 cover households: MOR0 and REN0; MOR1 and REN1; and MOR2 and REN2 respectively, for the 1980-85 four-region data. Similarly, the corresponding respective household groups are covered in Tables 14-16 for the 1980-92 four-region data; and in Tables 17-19 for the three-region data.

The first item to focus on in these tables is whether negativity of own-price elasticities is observed. Five positive own-price elasticities (out of a total of fifty-four own-price elasticities) are observed in Tables 11-13. Four of them are for renter households, but none of these are statistically significantly different from zero, based on the reported $Z$-ratios in the tables. Only the A category own-price elasticity for MOR1 households is positive and statistically significantly larger than zero.

With respect to income elasticities, F can be uniformly categorised as income inelastic for all household categories. G also has this property for all but the REN2 household group. Almost all other goods except E (personal care spending) are income elastic according to the Table 11-13 results.

On the whole, the Table 11-13 results exhibit plausible values for own-price and income elasticities. 
In addition, the elasticities are estimated with a high degree of precision. This is typically true of income elasticities when estimating demand models using cross-sectional microdata. However, the price data employed in the current study allow for improved precision in the estimation of price responses, as evidenced in Tables 11-13 by the generally high $Z$-ratios reported for price elasticities.

There are also substantial differences in own-price and income elasticities when comparing across family size, given housing tenure. For example, $\mathrm{F}$ takes own-price elasticity values of from -0.076 to -1.234 , for MOR0-MOR2 households, while C own-price elasticities range from -0.965 to -1.319 for the same households. When comparing elasticities across tenure status for a given household size, substantial differences are also observed. These types of findings lend strong support to the estimation of separate demand systems for households with differing sizes and housing tenures.

Tables 14-16 contain the corresponding estimated elasticities for the 1980-92 four-region data. Again, there is a total of five (out of fifty-four) own-price elasticities which are positive. In this case, none of the positive own-price elasticities are statistically significantly different from zero, based on the reported $Z$-ratios. It should be noted, however, that the 1980-92 four-region data contain errors in price variables. These errors could be introducing a degree of imprecision into the estimates, as well as certain biases.

To focus on the effects of the errors in the price variables, the results in Tables 14-16 can be compared with the corresponding results in Tables 11-13. With respect to income elasticities, one can again observe income inelastic demand for $\mathrm{F}$ and $\mathrm{G}$. Other income elasticities also exhibit the same kinds of properties across the two sets of results. On the other hand, with respect to price elasticities, particularly own-price elasticities, the differences in results are often substantial. For example, $\mathrm{F}$ is own-price elastic for MOR0 households in Table 11 and, own-price inelastic in Table 14. The converse is true for MOR1 households.

There is, of course, a trade-off in moving from Tables 11-13 versus Tables 14-16. That is, the former are free of the errors in price variables discussed earlier; on the other hand, these samples are smaller so less precise cetreris paribus, than the estimates in the latter Tables, based on larger samples. However, the latter results, even although they are based on larger sample sizes, will be affected by the systematic errors in the price variables. With this in mind, it is of interest to turn to the results based on the 1980-92 three-region data.

The 1980-92 three-region results corresponding to those in Tables 11-13 and Tables 14-16 are presented in Tables 17-19. With respect to own-price elasticities, ten out of a possible fifty-four estimates are positive. These are concentrated in the REN0-REN2 results, where eight of the "positives" occur. However, these 
aberrant values are not statistically significantly positive, based on the reported $Z$-ratios.

As with previous results, $\mathrm{F}$ and $\mathrm{G}$ are income inelastic, and other goods typically income elastic. For own-price elasticities, the Table 17-19 estimates typically confirm the qualitative values of these, relative to corresponding values in Tables 11-13. The precision of the former estimates are also superior to the latter, as is to be expected, given the larger sample sizes on which the Tables 17-19 results are based. Contrasting the three-region data results with those in Tables 14-16, there is usually fairly close agreement with regard to income elasticities, but there are often substantial differences between corresponding price elasticities. For example, the S (health care spending) own-price elasticities in Tables 11 and 17 indicate this category to be own-price inelastic for MOR0 households, yet Table 13 indicates a value of -1.541 - more than double the higher of the other two values $(-0.774$ and -0.476 for Tables 11 and 17 respectively). These kinds of contrasts would appear to support the argument that the errors in price variables have important effects, not only on the outcomes of hypothesis tests (as presented and discussed in the preceding sub-section), but also on estimates of more direct interest to the economist, such as elasticities.

To summarise, the extent to which elasticities take "sensible" values, particularly price elasticities, is encouraging, given the size of the system being estimated. This is possible only because of the significant amount of price variability in these data. In addition, the model is extremely general in terms of the influences which are being controlled for.

In most demand system estimation situations, one typically does not have the advantage of being able to estimate such a large system as one having nine equations. More typically, three, four or six equations are all that can be estimated, and various methods used to control and justify the estimation of the smaller system. The inclusion of a "conditioning good" (CG), such as g, defined in Section 2, is one approach. The next set of elasticities presented thus presents results based on the FACGOP system, which has six equations.

Tables 20-22 contain the estimated elasticities for MOR0-MOR2 households calculated using: 1980-85 four-region data, 1980-92 four-region data, and 1980-92 three-region data respectively, for the FACGOP system. In this case, there are seven, one and five own-price elasticities which are positive in each one of the three tables. As with the FACGOPHES results, the positive elasticities are concentrated in the renter household groups. Of these, only two are statistically significantly different from zero (there is a total of one-hundred and eight own-price elasticities in the tables).

Looking more closely at the elasticities themselves, F and $\mathrm{G}$ continue to be income inelastic for MOR0- 
MOR2 households, irrespective of which data are used. This is also true for renter households for F, but there are several instances for the renters where $\mathrm{G}$ appears to be income elastic. These cases occur with the 1980-85 four-region data. However, this pattern is absent when looking at the corresponding elasticities based on the 1980-92 three-region data. The patterns of income elasticities for other goods in this system are not as well-defined for most of the other goods, except $\mathrm{C}$, which is uniformly reported as income elastic for all household groups and data sets.

When contrasting own-price elasticities across Tables 21 and 22, with and without errors in price variables for MOR0-MOR2 households, there is often fairly close agreement in these values for some cases, but large differences for others. For example, A has an elasticity of -1.939 in Table 21 for MOR0 households, and -1.889 in Table 22. On the other hand, the same comparison for MOR2 households yields respective elasticities of -3.357 and -1.162 . Corresponding elasticity values from Table 20 are not particularly close to either of the Table 21 or 22 results, but the former estimates are not as precisely estimated, based on the values of the $Z$-ratios.

Of course, one might not expect a systematic pattern in the foregoing kinds of comparisons. That is, this demand system is a smaller one than FACGOPHES, and there is the possibility of influences associated with the $\mathrm{H}, \mathrm{E}$ and $\mathrm{S}$ expenditures being included in $g$, the conditioning good. To obtain an understanding of whether this is important, own-price elasticities from the FACGOPHES system can be compared with corresponding values from FACGOP system estimation.

Consider the estimates for the MOR2 household groups (there are no positive reported own-price elasticities for this group based on the FACGOP system). F is own-price inelastic according to the FACGOPHES results, no matter which of the three estimates one considers. However, the FACGOP results have F own-price elastic for two out of the three cases. Also, the 1980-92 four-region data results in Table 21 for MOR2 indicate $\mathrm{F}$ is income elastic, and elsewhere it is income inelastic (for other FACGOP estimates, and FACGOPHES estimates). Alternatively, $\mathrm{C}$ is reported as own-price inelastic in Table 16 (FACGOPHES with errors in price variables), while it is reported in Tables 13 and 19 as own-price elastic. This is also the case in Tables 20-22. Thus, the errors in price variables influence this elasticity in one direction, where errors associated with aggregation and separability in the opposite direction.

In general, as previously discussed, one is usually unable to estimate a system as large as FACGOPHES. The results suggest that extreme caution need be exercised in interpreting the results based on such estimation. This is highlighted even more when one moves to even smaller equation systems such as FAC, 


\section{GOP or HES.}

Estimated elasticities are presented for: the FAC system in Tables 26 and 27; the GOP system in Tables 28 and 29; and the HES system in Tables 30 and 31. Viewing these results as a whole, there are seventeen positive own-prices elasticities for the 1980-85 four-region data (as compared to five for the same results with the FACGOPHES system), fourteen positive own-price elasticities for the 1980-92 four-region data (five for the FACGOPHES system) and fifteen for the 1980-92 three-region data (ten for the FACGOPHES system). Clearly, performance on this criterion alone is much inferior to what was observed for the FACGOPHES system.

Not only are the reported positive own-price elasticities more numerous in Tables 26-31 than in Tables 11-19, but eleven of the forty-six are statistically significantly different from zero. These "positives" are evenly distributed across the two housing tenure classes, unlike the earlier results, where positives were concentrated in the renter household group.

The income elasticities in Tables 26-31 can also be compared with the corresponding FACGOPHES system results. In the present case, most results indicate income inelastic demand for F. However, there are three cases where $\mathrm{F}$ is income elastic (two of these are marginal). $\mathrm{G}$, however, is indicated to be income inelastic in all but one case.

Comparisons of price elasticities exhibit greater divergence from the FACGOPHES results. For example, the own-price elasticity for $\mathrm{F}$ is -0.206 for MOR0 households based on 1980-85 four-region data, whereas the corresponding value for the FACGOPHES system is -1.234. It should be noted, however, that the estimates arising from three-equation system estimation are, in general, much less precise than those for the FACGOPHES system. This is a little surprising, since many more parameters must be estimated with the latter (thirty-five parameters for a three-equation system; one-hundred and fifty-eight with the nine-equation system). However, additional information is added to the estimation environment along with the additional equations of the larger system. Evidently, this increased amount of information more than compensates for the increased number of parameters which have to be estimated. The loss in precision is most apparent in the HES system estimates of Tables 30 and 31 . Even when these estimates take economically plausible values, they often have large standard errors, as indicated by the reported $Z$-ratios.

Clearly, much more could be said with respect to individual comparisons of estimated elasticities across a range of dimensions. The main point which seems to emerge is that it is preferable to estimate as large and disaggregated a system of equations as possible. Even when there appears to be separability of 
goods included directly in a system from excluded goods, estimating conditional demands is a second-best solution.

Furthermore, it appears that errors in price variables not only affect test results, but have important effects on estimated elasticities. This will come as little of a surprise, but the results here represent a starting point for analysing the magnitudes of these effects, and suggests one might try to use the additional information on price variability which is sometimes available to control for these effects.

In conclusion, the evidence provided in this paper does not suggest that a demand system specification can be developed in a way where "one size fits all". That is, there are important differences in demand patterns and the determinants of these patterns across households which cannot be assumed away. Findings that a particular model works well in the context of a data set comprising homogeneous households does not mean that the same model will perform equally well for other household groups. In particular, while demands might appear to be rank three for a given household group, as has been seen, this cannot be assumed to be generally true. In addition, while labour force participation variables are important determinants of demand for the MOR0-MOR2 households identified in this paper, this does not appear to be true for REN0-REN2 households. The only evidence in favour of this hypothesis was obtained when significant errors in the variables being used were present.

\section{$5 \quad$ Summary and Conclusions}

In this paper, a rank three demand system was estimated. The model was specified to control for the possibility of non-separability of labour force participation effects, non-separability of other goods not included directly in the demand system, and the influence of a variety of household characteristics effects. Systems with from three equations to nine equations were estimated, for six different household types. Given the nature of the data available, it was also possible to analyse the effects of errors in price variables on the outcome of hypothesis tests and estimated elasticities.

The possible rank three nature of demands appeared to be significantly affected by the presence of errors in price variables. Test results in the absence of these errors indicated little evidence in support of the rank three hypothesis. This is in contrast to other research, for example, Banks, Blundell and Lewbel (1997). However, in that case, there is the distinct possibility of large, systematic errors in the price variables used. This issue is one which merits further investigation.

Various other influences such as labour force participation were also found to be important determinants of demand. However, this finding was not universal across the range of different households considered 
in this study. Again, there was some indication of the effect of errors in variables on these test results. The conclusion appears to be that there is not completely general model of demand which applies to all households of different types, even where differences in corresponding parameters across these systems are permitted.

Estimated elasticities were also computed for the different models. The values arising from the largest system estimated (nine equations) were extremely encouraging, with elasticities taking plausible magnitudes in most cases. There was evidence, however, that the presence of errors in price variables significantly influenced the elasticities. In addition, the stability of the estimates was much better with relatively larger systems compared to smaller ones. However, it is not usually possible to estimate systems as large as the nine-equation system of this study, owing to difficulties in empirically identifying all parameters of such a system, given the limited amount of information typically present in available price data.

The data used in this study were selected from a series of US CES surveys. However, not all available data were used in this particular application. The results obtained here give guidance for some future research. In particular, for certain household types, some variables included as determinants of demand here were not found to be statistically significant. Also, the smaller disaggregated sub-systems of demand did not perform nearly as well as the larger system. Errors in price variables were also seen to be important. In future work, these factors can be taken into account in obtaining more precise estimates of parameters of interest, elasticities, equivalence scales, and other demand related magnitudes available as by-products of demand system estimation. This research is ongoing.

\section{References}

American Chamber of Commerce Researchers Association, Inter-City Price Indices for the United States. Various years.

Banks, J., R. Blundell and A. Lewbel (1992), "Kernel Regression, Quadratric Logarithmic Engel Curves and Welfare Measurement." Institute for Fiscal Studies Working Paper No. 92/14.

Banks, J., R. Blundell and A. Lewbel (1997), "Quadratic Engel Curves and Consumer Demand." The Review of Economics and Statistics, 79, 527-539.

Barnes, R. and R. Gillingham (1984), "Demographic Effects in Demand Analysis: Estimation of the Quadratic Expenditure System." Review of Economics and Statistics, 66, 591-601. 
Blundell, R., P. Pashardes and G. Weber (1993), "What Do We Learn About Consumer Demand Patterns From Micro Data." American Economic Review, 83, 570-597.

Browning, M. (1983), "Necessary and Sufficient Conditions for Conditional Cost Functions." Econometrica, 51, 851-856.

Browning, M. and C. Meghir (1991), "The Effects of Male and Female Labor Supply on Commodity Demands." Econometrica, 59, 925-951.

Bureau of Labor Statistics, Washington DC. Interview Survey Public-Use Tapes, Consumer Expenditure Surveys, 1980-1992.

Cragg, J. G. (1994), "Innis Lecture: Making Good Inferences from Bad Data." Canadian Journal of Economics, 27, 776-800.

Dagenais, M. (1994), "Parameter Estimation in Regression Models With Errors in Variables and Autocorrelated Disturbances." Journal of Econometrics 64, 145-163.

Deaton, A. and J. Muellbauer (1980), “An Almost Ideal Demand System.” American Economic Review, 70, 312-326.

Dickens, R., V. Fry and P. Pashardes (1993), "Non-linearities and Equivalence Scales." Economic Journal, 103, 359-368.

Fry, V. and P. Pashardes (1992), "An Almost Ideal Quadratic Logarithmic Demand System for the Analysis of Micro Data." Discussion Paper No. 25, City University of London.

Howe, H., R. Pollak and T. Wales (1978), "Theory and Time-Series Estimation of the Quadratic Expenditure System." Econometrica, 47, 1231-1247.

Kaiser, H. (1993), "Testing for Separability Between Commodity Demand and Labour Supply in West Germany." 18, 21-56.

Lewbel, A. (1990), "Full Rank Demand Systems." International Economic Review, 31, 289-300.

Lewbel, A. (1991), "The Rank of Demand Systems: Theory and Non-parametric Estimation." Econometrica, 59, 711-730. 
Nicol, C.J. (1989), "Testing a Theory of Exact Aggregation.” Journal of Business and Economic Statistics, $7,259-265$.

Nicol, C.J. (1991), “The Effect of Expenditure Aggregation on Hypothesis Tests in Consumer Demand Systems." International Economic Review, 32, 405-416.

Nicol, C.J. (1995) "Model Specification Issues in Consumer Demand Systems Using United States Microdata". Department of Economics Working Paper No. 56, University of Regina.

Pollak, R. (1969), "Conditional Demand Functions and the Implications of Separability." Quarterly Journal of Economics, 83, 70-78.

van Daal, J. and A. H. Q. M. Mierkes (1989), “A Note on the Quadratic Expenditure Model." Econometrica, 57, 1439-1443. 


\section{Notes to Tables 1-10}

1. In the headings to the test statistics, "Excluding Q" and so on indicates the variables being excluded are: Q, quadratic terms in total expenditures; A, age of head; L, labour force variables; C, other expenditures conditioning good; $\mathrm{V}$, vehicle ownership; and $\mathrm{T}$, tobacco consumption dummy.

2. All test statistics are disributed as $\chi^{2}$ under the null hypothesis.

3. The degrees of freedom for the nine nine equation system (FACGOPHES) tests are 32 for L; 16 for A, $\mathrm{C}, \mathrm{V}$ and $\mathrm{T}$; and 9 for $\mathrm{Q}$.

4. The degrees of freedom for the six equation system (FACGOP) tests tests are 20 for L; 10 for A, C, V and $\mathrm{T}$; and 6 for $\mathrm{Q}$.

5. The degrees of freedom for the three-equation systems (FAC, GOP and HES) tests are: 8 for L; 4 for $\mathrm{A}, \mathrm{C}, \mathrm{V}$ and $\mathrm{T}$; and 3 for $\mathrm{Q}$. 
Table 1: Model Specification Tests Based on GMM Estimation.

FACGOPHES System

MOR0, MOR1 and MOR2 Households.

Tests Based on Estimation with Four-Region Data, 1980-1992.

\begin{tabular}{|c|c|c|c|c|c|c|}
\hline \multirow[b]{2}{*}{ Data } & \multicolumn{2}{|c|}{ Excluding Q } & \multicolumn{2}{|c|}{ Excluding A } & \multicolumn{2}{|c|}{ Excluding L } \\
\hline & Test Statistic & Prob. Value & Test Statistic & Prob. Value & Test Statistic & Prob. Value \\
\hline MOR0 & 47.766 & $0.282 \mathrm{E}-06$ & 130.797 & $0.000 \mathrm{E}+00$ & 118.993 & $0.610 \mathrm{E}-11$ \\
\hline MOR1 & 80.950 & $0.105 \mathrm{E}-12$ & 146.830 & $0.000 \mathrm{E}+00$ & 165.133 & $0.000 \mathrm{E}+00$ \\
\hline MOR2 & 98.477 & $0.000 \mathrm{E}+00$ & 262.046 & $0.000 \mathrm{E}+00$ & 238.342 & $0.000 \mathrm{E}+00$ \\
\hline \multirow[b]{2}{*}{ Data } & \multicolumn{2}{|c|}{ Excluding $\mathrm{C}$} & \multicolumn{2}{|c|}{ Excluding V } & \multicolumn{2}{|c|}{ Excluding $\mathrm{T}$} \\
\hline & Test Statistic & Prob. Value & Test Statistic & Prob. Value & Test Statistic & Prob. Value \\
\hline MOR0 & 81.393 & $0.932 \mathrm{E}-10$ & 73.780 & $0.215 \mathrm{E}-08$ & 38.088 & $0.147 \mathrm{E}-02$ \\
\hline MOR1 & 192.684 & $0.000 \mathrm{E}+00$ & 114.966 & $0.000 \mathrm{E}+00$ & 160.252 & $0.000 \mathrm{E}+00$ \\
\hline MOR2 & 212.426 & $0.000 \mathrm{E}+00$ & 111.669 & $0.222 \mathrm{E}-15$ & 57.667 & $0.129 \mathrm{E}-05$ \\
\hline
\end{tabular}

Tests Based on Estimation with Four-Region Data, 1980-1985.

\begin{tabular}{|c|c|c|c|c|c|c|}
\hline \multirow[b]{2}{*}{ Data } & \multicolumn{2}{|c|}{ Excluding Q } & \multicolumn{2}{|c|}{ Excluding A } & \multicolumn{2}{|c|}{ Excluding L } \\
\hline & Test Statistic & Prob. Value & Test Statistic & Prob. Value & Test Statistic & Prob. Value \\
\hline MOR0 & 24.520 & $0.355 \mathrm{E}-02$ & 81.655 & $0.836 \mathrm{E}-10$ & 107.109 & $0.496 \mathrm{E}-09$ \\
\hline MOR1 & 5.794 & $0.760 \mathrm{E}+00$ & 232.062 & $0.000 \mathrm{E}+00$ & 293.640 & $0.000 \mathrm{E}+00$ \\
\hline MOR2 & 10.971 & $0.278 \mathrm{E}+00$ & 114.312 & $0.111 \mathrm{E}-15$ & 209.618 & $0.000 \mathrm{E}+00$ \\
\hline \multirow[b]{2}{*}{ Data } & \multicolumn{2}{|c|}{ Excluding C } & \multicolumn{2}{|c|}{ Excluding V } & \multicolumn{2}{|c|}{ Excluding $\mathrm{T}$} \\
\hline & Test Statistic & Prob. Value & Test Statistic & Prob. Value & Test Statistic & Prob. Value \\
\hline MOR0 & 142.743 & $0.000 \mathrm{E}+00$ & 32.188 & $0.945 \mathrm{E}-02$ & 30.725 & $0.146 \mathrm{E}-01$ \\
\hline MOR1 & 72.610 & $0.347 \mathrm{E}-08$ & 51.940 & $0.112 \mathrm{E}-04$ & 40.690 & $0.617 \mathrm{E}-03$ \\
\hline MOR2 & 108.207 & $0.999 \mathrm{E}-15$ & 45.171 & $0.131 \mathrm{E}-03$ & 58.827 & $0.824 \mathrm{E}-06$ \\
\hline
\end{tabular}

Tests Based on Estimation with Three-Region Data, 1980-1992.

\begin{tabular}{|c|c|c|c|c|c|c|}
\hline \multirow[b]{2}{*}{ Data } & \multicolumn{2}{|c|}{ Excluding Q } & \multicolumn{2}{|c|}{ Excluding A } & \multicolumn{2}{|c|}{ Excluding L } \\
\hline & Test Statistic & Prob. Value & Test Statistic & Prob. Value & Test Statistic & Prob. Value \\
\hline MOR0 & 10.203 & $0.334 \mathrm{E}+00$ & 141.269 & $0.000 \mathrm{E}+00$ & 100.827 & $0.474 \mathrm{E}-08$ \\
\hline MOR1 & 46.055 & $0.588 \mathrm{E}-06$ & 144.220 & $0.000 \mathrm{E}+00$ & 156.499 & $0.000 \mathrm{E}+00$ \\
\hline MOR2 & 20.198 & $0.167 \mathrm{E}-01$ & 204.538 & $0.000 \mathrm{E}+00$ & 97.507 & $0.153 \mathrm{E}-07$ \\
\hline \multirow[b]{2}{*}{ Data } & \multicolumn{2}{|c|}{ Excluding $\mathrm{C}$} & \multicolumn{2}{|c|}{ Excluding V } & \multicolumn{2}{|c|}{ Excluding $\mathrm{T}$} \\
\hline & Test Statistic & Prob. Value & Test Statistic & Prob. Value & Test Statistic & Prob. Value \\
\hline MOR0 & 39.642 & $0.878 \mathrm{E}-03$ & 23.173 & $0.109 \mathrm{E}+00$ & 42.059 & $0.387 \mathrm{E}-03$ \\
\hline MOR1 & 203.865 & $0.000 \mathrm{E}+00$ & 122.450 & $0.000 \mathrm{E}+00$ & 92.344 & $0.924 \mathrm{E}-12$ \\
\hline MOR2 & 71.507 & $0.543 \mathrm{E}-08$ & 47.220 & $0.629 \mathrm{E}-04$ & 51.517 & $0.131 \mathrm{E}-04$ \\
\hline
\end{tabular}


Table 2: Model Specification Tests Based on GMM Estimation.

FACGOPHES System

REN0, REN1 and REN2 Households.

Tests Based on Estimation with Four-Region Data, 1980-1992.

\begin{tabular}{|c|c|c|c|c|c|c|}
\hline \multirow[b]{2}{*}{ Data } & \multicolumn{2}{|c|}{ Excluding Q } & \multicolumn{2}{|c|}{ Excluding A } & \multicolumn{2}{|c|}{ Excluding L } \\
\hline & Test Statistic & Prob. Value & Test Statistic & Prob. Value & Test Statistic & Prob. Value \\
\hline REN0 & 125.164 & $0.000 \mathrm{E}+00$ & 121.758 & $0.000 \mathrm{E}+00$ & 385.489 & $0.000 \mathrm{E}+00$ \\
\hline REN1 & 95.330 & $0.111 \mathrm{E}-15$ & 80.694 & $0.125 \mathrm{E}-09$ & 185.985 & $0.000 \mathrm{E}+00$ \\
\hline REN2 & 221.531 & $0.000 \mathrm{E}+00$ & 788.333 & $0.000 \mathrm{E}+00$ & 547.868 & $0.000 \mathrm{E}+00$ \\
\hline & \multicolumn{2}{|c|}{ Excluding $\mathrm{C}$} & \multicolumn{2}{|c|}{ Excluding V } & \multicolumn{2}{|c|}{ Excluding $\mathrm{T}$} \\
\hline Data & Test Statistic & Prob. Value & Test Statistic & Prob. Value & Test Statistic & Prob. Value \\
\hline REN0 & 119.640 & $0.000 \mathrm{E}+00$ & 227.570 & $0.000 \mathrm{E}+00$ & 130.850 & $0.000 \mathrm{E}+00$ \\
\hline REN1 & 40.973 & $0.560 \mathrm{E}-03$ & 115.840 & $0.000 \mathrm{E}+00$ & 90.674 & $0.188 \mathrm{E}-11$ \\
\hline REN2 & 236.154 & $0.000 \mathrm{E}+00$ & 376.419 & $0.000 \mathrm{E}+00$ & 238.552 & $0.000 \mathrm{E}+00$ \\
\hline
\end{tabular}

Tests Based on Estimation with Four-Region Data, 1980-1985.

\begin{tabular}{|c|c|c|c|c|c|c|}
\hline \multirow[b]{2}{*}{ Data } & \multicolumn{2}{|c|}{ Excluding Q } & \multicolumn{2}{|c|}{ Excluding A } & \multicolumn{2}{|c|}{ Excluding L } \\
\hline & Test Statistic & Prob. Value & Test Statistic & Prob. Value & Test Statistic & Prob. Value \\
\hline REN0 & 10.299 & $0.327 \mathrm{E}+00$ & 13.668 & $0.623 \mathrm{E}+00$ & 43.854 & $0.790 \mathrm{E}-01$ \\
\hline REN1 & 0.254 & $0.100 \mathrm{E}+01$ & 17.575 & $0.349 \mathrm{E}+00$ & 52.073 & $0.139 \mathrm{E}-01$ \\
\hline REN2 & 9.271 & $0.413 \mathrm{E}+00$ & 36.716 & $0.230 \mathrm{E}-02$ & 53.643 & $0.963 \mathrm{E}-02$ \\
\hline \multirow[b]{2}{*}{ Data } & \multicolumn{2}{|c|}{ Excluding $\mathrm{C}$} & \multicolumn{2}{|c|}{ Excluding V } & \multicolumn{2}{|c|}{ Excluding $\mathrm{T}$} \\
\hline & Test Statistic & Prob. Value & Test Statistic & Prob. Value & Test Statistic & Prob. Value \\
\hline REN0 & 11.228 & $0.795 \mathrm{E}+00$ & 22.067 & $0.141 \mathrm{E}+00$ & 34.750 & $0.430 \mathrm{E}-02$ \\
\hline REN1 & 4.191 & $0.999 \mathrm{E}+00$ & 23.162 & $0.109 \mathrm{E}+00$ & 16.012 & $0.452 \mathrm{E}+00$ \\
\hline REN2 & 15.674 & $0.476 \mathrm{E}+00$ & 48.500 & $0.396 \mathrm{E}-04$ & 22.116 & $0.139 \mathrm{E}+00$ \\
\hline
\end{tabular}

Tests Based on Estimation with Three-Region Data, 1980-1992.

\begin{tabular}{|c|c|c|c|c|c|c|}
\hline \multirow[b]{2}{*}{ Data } & \multicolumn{2}{|c|}{ Excluding Q } & \multicolumn{2}{|c|}{ Excluding A } & \multicolumn{2}{|c|}{ Excluding L } \\
\hline & Test Statistic & Prob. Value & Test Statistic & Prob. Value & Test Statistic & Prob. Value \\
\hline REN0 & 3.227 & $0.955 \mathrm{E}+00$ & 21.826 & $0.149 \mathrm{E}+00$ & 48.632 & $0.301 \mathrm{E}-01$ \\
\hline REN1 & 9.408 & $0.400 \mathrm{E}+00$ & 15.590 & $0.482 \mathrm{E}+00$ & 47.822 & $0.357 \mathrm{E}-01$ \\
\hline REN2 & 14.338 & $0.111 \mathrm{E}+00$ & 26.783 & $0.440 \mathrm{E}-01$ & 45.661 & $0.556 \mathrm{E}-01$ \\
\hline \multirow[b]{2}{*}{ Data } & \multicolumn{2}{|c|}{ Excluding $\mathrm{C}$} & \multicolumn{2}{|c|}{ Excluding $\mathrm{V}$} & \multicolumn{2}{|c|}{ Excluding $\mathrm{T}$} \\
\hline & Test Statistic & Prob. Value & Test Statistic & Prob. Value & Test Statistic & Prob. Value \\
\hline REN0 & 18.558 & $0.292 \mathrm{E}+00$ & 37.768 & $0.163 \mathrm{E}-02$ & 36.201 & $0.271 \mathrm{E}-02$ \\
\hline REN1 & 11.383 & $0.785 \mathrm{E}+00$ & 12.757 & $0.690 \mathrm{E}+00$ & 10.489 & $0.840 \mathrm{E}+00$ \\
\hline REN2 & 18.494 & $0.296 \mathrm{E}+00$ & 49.468 & $0.279 \mathrm{E}-04$ & 8.704 & $0.925 \mathrm{E}+00$ \\
\hline
\end{tabular}


Table 3: Model Specification Tests Based on GMM Estimation.

FACGOP System

MOR0, MOR1 and MOR2 Households.

Tests Based on Estimation with Four-Region Data, 1980-1992.

\begin{tabular}{|c|c|c|c|c|c|c|}
\hline \multirow[b]{2}{*}{ Data } & \multicolumn{2}{|c|}{ Excluding Q } & \multicolumn{2}{|c|}{ Excluding A } & \multicolumn{2}{|c|}{ Excluding L } \\
\hline & Test Statistic & Prob. Value & Test Statistic & Prob. Value & Test Statistic & Prob. Value \\
\hline MOR0 & 36.233 & $0.248 \mathrm{E}-05$ & 63.306 & $0.854 \mathrm{E}-09$ & 27.253 & $0.128 \mathrm{E}+00$ \\
\hline MOR1 & 73.523 & $0.773 \mathrm{E}-13$ & 36.116 & $0.804 \mathrm{E}-04$ & 204.586 & $0.000 \mathrm{E}+00$ \\
\hline MOR2 & 4.204 & $0.649 \mathrm{E}+00$ & 26.628 & $0.298 \mathrm{E}-02$ & 70.204 & $0.169 \mathrm{E}-06$ \\
\hline \multirow[b]{2}{*}{ Data } & \multicolumn{2}{|c|}{ Excluding $\mathrm{C}$} & \multicolumn{2}{|c|}{ Excluding V } & \multicolumn{2}{|c|}{ Excluding $T$} \\
\hline & Test Statistic & Prob. Value & Test Statistic & Prob. Value & Test Statistic & Prob. Value \\
\hline MOR0 & 35.363 & $0.108 \mathrm{E}-03$ & 17.193 & $0.702 \mathrm{E}-01$ & 38.462 & $0.315 \mathrm{E}-04$ \\
\hline MOR1 & 65.551 & $0.318 \mathrm{E}-09$ & 64.342 & $0.541 \mathrm{E}-09$ & 77.579 & $0.149 \mathrm{E}-11$ \\
\hline MOR2 & 46.522 & $0.115 \mathrm{E}-05$ & 39.646 & $0.196 \mathrm{E}-04$ & 50.692 & $0.199 \mathrm{E}-06$ \\
\hline
\end{tabular}

Tests Based on Estimation with Four-Region Data, 1980-1985.

\begin{tabular}{|c|c|c|c|c|c|c|}
\hline \multirow[b]{2}{*}{ Data } & \multicolumn{2}{|c|}{ Excluding Q } & \multicolumn{2}{|c|}{ Excluding A } & \multicolumn{2}{|c|}{ Excluding L } \\
\hline & Test Statistic & Prob. Value & Test Statistic & Prob. Value & Test Statistic & Prob. Value \\
\hline MOR0 & 3.130 & $0.792 \mathrm{E}+00$ & 36.741 & $0.627 \mathrm{E}-04$ & 31.461 & $0.494 \mathrm{E}-01$ \\
\hline MOR1 & 0.466 & $0.998 \mathrm{E}+00$ & 58.717 & $0.633 \mathrm{E}-08$ & 68.522 & $0.317 \mathrm{E}-06$ \\
\hline MOR2 & 18.216 & $0.571 \mathrm{E}-02$ & 35.216 & $0.115 \mathrm{E}-03$ & 76.143 & $0.176 \mathrm{E}-07$ \\
\hline \multirow[b]{2}{*}{ Data } & \multicolumn{2}{|c|}{ Excluding C } & \multicolumn{2}{|c|}{ Excluding V } & \multicolumn{2}{|c|}{ Excluding $T$} \\
\hline & Test Statistic & Prob. Value & Test Statistic & Prob. Value & Test Statistic & Prob. Value \\
\hline MOR0 & 25.581 & $0.435 \mathrm{E}-02$ & 10.473 & $0.400 \mathrm{E}+00$ & 36.848 & $0.601 \mathrm{E}-04$ \\
\hline MOR1 & 24.322 & $0.679 \mathrm{E}-02$ & 32.348 & $0.350 \mathrm{E}-03$ & 13.421 & $0.201 \mathrm{E}+00$ \\
\hline MOR2 & 33.032 & $0.269 \mathrm{E}-03$ & 11.854 & $0.295 \mathrm{E}+00$ & 8.852 & $0.546 \mathrm{E}+00$ \\
\hline
\end{tabular}

Tests Based on Estimation with Three-Region Data, 1980-1992.

\begin{tabular}{|c|c|c|c|c|c|c|}
\hline \multirow[b]{2}{*}{ Data } & \multicolumn{2}{|c|}{ Excluding Q } & \multicolumn{2}{|c|}{ Excluding A } & \multicolumn{2}{|c|}{ Excluding L } \\
\hline & Test Statistic & Prob. Value & Test Statistic & Prob. Value & Test Statistic & Prob. Value \\
\hline MOR0 & 57.153 & $0.170 \mathrm{E}-09$ & 55.609 & $0.243 \mathrm{E}-07$ & 30.583 & $0.609 \mathrm{E}-01$ \\
\hline MOR1 & 69.061 & $0.637 \mathrm{E}-12$ & 26.500 & $0.312 \mathrm{E}-02$ & 103.355 & $0.315 \mathrm{E}-12$ \\
\hline MOR2 & 0.233 & $0.100 \mathrm{E}+01$ & 23.263 & $0.982 \mathrm{E}-02$ & 40.829 & $0.392 \mathrm{E}-02$ \\
\hline \multirow[b]{2}{*}{ Data } & \multicolumn{2}{|c|}{ Excluding $\mathrm{C}$} & \multicolumn{2}{|c|}{ Excluding V } & \multicolumn{2}{|c|}{ Excluding $\mathrm{T}$} \\
\hline & Test Statistic & Prob. Value & Test Statistic & Prob. Value & Test Statistic & Prob. Value \\
\hline MOR0 & 32.730 & $0.302 \mathrm{E}-03$ & 11.908 & $0.291 \mathrm{E}+00$ & 25.291 & $0.482 \mathrm{E}-02$ \\
\hline MOR1 & 38.687 & $0.288 \mathrm{E}-04$ & 182.689 & $0.000 \mathrm{E}+00$ & 68.075 & $0.104 \mathrm{E}-09$ \\
\hline MOR2 & 32.760 & $0.299 \mathrm{E}-03$ & 34.610 & $0.145 \mathrm{E}-03$ & 20.921 & $0.217 \mathrm{E}-01$ \\
\hline
\end{tabular}


Table 4: Model Specification Tests Based on GMM Estimation.

FACGOP System

REN0, REN1 and REN2 Households.

Tests Based on Estimation with Four-Region Data, 1980-1992.

\begin{tabular}{|c|c|c|c|c|c|c|}
\hline \multirow[b]{2}{*}{ Data } & \multicolumn{2}{|c|}{ Excluding Q } & \multicolumn{2}{|c|}{ Excluding A } & \multicolumn{2}{|c|}{ Excluding L } \\
\hline & Test Statistic & Prob. Value & Test Statistic & Prob. Value & Test Statistic & Prob. Value \\
\hline REN0 & 13.423 & $0.368 \mathrm{E}-01$ & 53.806 & $0.527 \mathrm{E}-07$ & 26.037 & $0.165 \mathrm{E}+00$ \\
\hline REN1 & 20.381 & $0.237 \mathrm{E}-02$ & 27.704 & $0.201 \mathrm{E}-02$ & 41.492 & $0.322 \mathrm{E}-02$ \\
\hline REN2 & 0.322 & $0.999 \mathrm{E}+00$ & 21.664 & $0.169 \mathrm{E}-01$ & 24.003 & $0.242 \mathrm{E}+00$ \\
\hline \multirow[b]{2}{*}{ Data } & \multicolumn{2}{|c|}{ Excluding C } & \multicolumn{2}{|c|}{ Excluding V } & \multicolumn{2}{|c|}{ Excluding $\mathrm{T}$} \\
\hline & Test Statistic & Prob. Value & Test Statistic & Prob. Value & Test Statistic & Prob. Value \\
\hline REN0 & 19.844 & $0.308 \mathrm{E}-01$ & 41.586 & $0.888 \mathrm{E}-05$ & 39.775 & $0.186 \mathrm{E}-04$ \\
\hline REN1 & 25.545 & $0.440 \mathrm{E}-02$ & 19.853 & $0.307 \mathrm{E}-01$ & 42.014 & $0.746 \mathrm{E}-05$ \\
\hline REN2 & 7.619 & $0.666 \mathrm{E}+00$ & 46.474 & $0.118 \mathrm{E}-05$ & 5.199 & $0.878 \mathrm{E}+00$ \\
\hline
\end{tabular}

Tests Based on Estimation with Four-Region Data, 1980-1985.

\begin{tabular}{|c|c|c|c|c|c|c|}
\hline \multirow[b]{2}{*}{ Data } & \multicolumn{2}{|c|}{ Excluding Q } & \multicolumn{2}{|c|}{ Excluding A } & \multicolumn{2}{|c|}{ Excluding L } \\
\hline & Test Statistic & Prob. Value & Test Statistic & Prob. Value & Test Statistic & Prob. Value \\
\hline REN0 & 20.391 & $0.236 \mathrm{E}-02$ & 33.943 & $0.189 \mathrm{E}-03$ & 73.707 & $0.447 \mathrm{E}-07$ \\
\hline REN1 & 0.034 & $0.100 \mathrm{E}+01$ & 12.121 & $0.277 \mathrm{E}+00$ & 13.634 & $0.849 \mathrm{E}+00$ \\
\hline REN2 & 5.830 & $0.442 \mathrm{E}+00$ & 15.207 & $0.125 \mathrm{E}+00$ & 19.741 & $0.474 \mathrm{E}+00$ \\
\hline \multirow[b]{2}{*}{ Data } & \multicolumn{2}{|c|}{ Excluding $\mathrm{C}$} & \multicolumn{2}{|c|}{ Excluding V } & \multicolumn{2}{|c|}{ Excluding $\mathrm{T}$} \\
\hline & Test Statistic & Prob. Value & Test Statistic & Prob. Value & Test Statistic & Prob. Value \\
\hline REN0 & 49.318 & $0.356 \mathrm{E}-06$ & 101.871 & $0.000 \mathrm{E}+00$ & 37.962 & $0.385 \mathrm{E}-04$ \\
\hline REN1 & 6.298 & $0.790 \mathrm{E}+00$ & 23.503 & $0.904 \mathrm{E}-02$ & 10.110 & $0.431 \mathrm{E}+00$ \\
\hline REN2 & 4.277 & $0.934 \mathrm{E}+00$ & 35.486 & $0.103 \mathrm{E}-03$ & 14.126 & $0.167 \mathrm{E}+00$ \\
\hline
\end{tabular}

Tests Based on Estimation with Three-Region Data, 1980-1992.

\begin{tabular}{|c|c|c|c|c|c|c|}
\hline \multirow[b]{2}{*}{ Data } & \multicolumn{2}{|c|}{ Excluding Q } & \multicolumn{2}{|c|}{ Excluding A } & \multicolumn{2}{|c|}{ Excluding L } \\
\hline & Test Statistic & Prob. Value & Test Statistic & Prob. Value & Test Statistic & Prob. Value \\
\hline REN0 & 13.877 & $0.310 \mathrm{E}-01$ & 57.792 & $0.946 \mathrm{E}-08$ & 47.888 & $0.441 \mathrm{E}-03$ \\
\hline REN1 & 3.414 & $0.755 \mathrm{E}+00$ & 70.577 & $0.343 \mathrm{E}-10$ & 66.453 & $0.683 \mathrm{E}-06$ \\
\hline REN2 & 16.437 & $0.116 \mathrm{E}-01$ & 19.803 & $0.312 \mathrm{E}-01$ & 55.882 & $0.303 \mathrm{E}-04$ \\
\hline \multirow[b]{2}{*}{ Data } & \multicolumn{2}{|c|}{ Excluding C } & \multicolumn{2}{|c|}{ Excluding $\mathrm{V}$} & \multicolumn{2}{|c|}{ Excluding $\mathrm{T}$} \\
\hline & Test Statistic & Prob. Value & Test Statistic & Prob. Value & Test Statistic & Prob. Value \\
\hline REN0 & 20.327 & $0.263 \mathrm{E}-01$ & 18.136 & $0.527 \mathrm{E}-01$ & 40.248 & $0.153 \mathrm{E}-04$ \\
\hline REN1 & 35.411 & $0.106 \mathrm{E}-03$ & 22.277 & $0.138 \mathrm{E}-01$ & 55.094 & $0.303 \mathrm{E}-07$ \\
\hline REN2 & 19.618 & $0.331 \mathrm{E}-01$ & 69.212 & $0.629 \mathrm{E}-10$ & 19.836 & $0.308 \mathrm{E}-01$ \\
\hline
\end{tabular}


Table 5: Model Specification Tests Based on GMM Estimation FAC System

MOR0, MOR1 and MOR2 Households.

Tests Based on Estimation with Four-Region Data, 1980-1992.

\begin{tabular}{|c|c|c|c|c|c|c|}
\hline \multirow[b]{2}{*}{ Data } & \multicolumn{2}{|c|}{ Excluding $\mathrm{Q}$} & \multicolumn{2}{|c|}{ Excluding A } & \multicolumn{2}{|c|}{ Excluding L } \\
\hline & Test Statistic & Prob. Value & Test Statistic & Prob. Value & Test Statistic & Prob. Value \\
\hline MOR0 & 22.821 & $0.440 \mathrm{E}-04$ & 15.300 & $0.412 \mathrm{E}-02$ & 82.622 & $0.145 \mathrm{E}-13$ \\
\hline MOR1 & 1.765 & $0.623 \mathrm{E}+00$ & 9.706 & $0.457 \mathrm{E}-01$ & 3.009 & $0.934 \mathrm{E}+00$ \\
\hline \multirow[t]{2}{*}{ MOR2 } & 12.689 & $0.536 \mathrm{E}-02$ & 20.496 & $0.399 \mathrm{E}-03$ & 7.822 & $0.451 \mathrm{E}+00$ \\
\hline & \multicolumn{2}{|c|}{ Excluding $\mathrm{C}$} & \multicolumn{2}{|c|}{ Excluding $\mathrm{V}$} & \multicolumn{2}{|c|}{ Excluding $\mathrm{T}$} \\
\hline Data & Test Statistic & Prob. Value & Test Statistic & Prob. Value & Test Statistic & Prob. Value \\
\hline MOR0 & 18.491 & $0.989 \mathrm{E}-03$ & 43.104 & $0.985 \mathrm{E}-08$ & 2.723 & $0.605 \mathrm{E}+00$ \\
\hline MOR1 & 19.017 & $0.780 \mathrm{E}-03$ & 12.468 & $0.142 \mathrm{E}-01$ & 3.033 & $0.552 \mathrm{E}+00$ \\
\hline MOR2 & 14.883 & $0.495 \mathrm{E}-02$ & 2.090 & $0.719 \mathrm{E}+00$ & .695 & $0.952 \mathrm{E}+00$ \\
\hline
\end{tabular}

Tests Based on Estimation with Four-Region Data, 1980-1985.

\begin{tabular}{|c|c|c|c|c|c|c|}
\hline \multirow[b]{2}{*}{ Data } & \multicolumn{2}{|c|}{ Excluding $\mathrm{Q}$} & \multicolumn{2}{|c|}{ Excluding $\mathrm{A}$} & \multicolumn{2}{|c|}{ Excluding L } \\
\hline & Test Statistic & Prob. Value & Test Statistic & Prob. Value & Test Statistic & Prob. Value \\
\hline MOR0 & 0.956 & $0.812 \mathrm{E}+00$ & 3.478 & $0.481 \mathrm{E}+00$ & 15.926 & $0.435 \mathrm{E}-01$ \\
\hline MOR1 & 0.041 & $0.998 \mathrm{E}+00$ & 4.422 & $0.352 \mathrm{E}+00$ & 9.840 & $0.276 \mathrm{E}+00$ \\
\hline \multirow[t]{2}{*}{ MOR2 } & 0.055 & $0.997 \mathrm{E}+00$ & 0.882 & $0.927 \mathrm{E}+00$ & 11.855 & $0.158 \mathrm{E}+00$ \\
\hline & \multicolumn{2}{|c|}{ Excluding $\mathrm{C}$} & \multicolumn{2}{|c|}{ Excluding $\mathrm{V}$} & \multicolumn{2}{|c|}{ Excluding $\mathrm{T}$} \\
\hline Data & Test Statistic & Prob. Value & Test Statistic & Prob. Value & Test Statistic & Prob. Value \\
\hline MOR0 & 4.406 & $0.354 \mathrm{E}+00$ & 0.996 & $0.910 \mathrm{E}+00$ & 3.054 & $0.549 \mathrm{E}+00$ \\
\hline MOR1 & 2.702 & $0.609 \mathrm{E}+00$ & 1.323 & $0.857 \mathrm{E}+00$ & 0.626 & $0.960 \mathrm{E}+00$ \\
\hline MOR2 & 12.475 & $0.141 \mathrm{E}-01$ & 0.935 & $0.920 \mathrm{E}+00$ & 5.615 & $0.230 \mathrm{E}+00$ \\
\hline
\end{tabular}

Tests Based on Estimation with Three-Region Data, 1980-1992.

\begin{tabular}{|c|c|c|c|c|c|c|}
\hline \multirow[b]{2}{*}{ Data } & \multicolumn{2}{|c|}{ Excluding $\mathrm{Q}$} & \multicolumn{2}{|c|}{ Excluding $\mathrm{A}$} & \multicolumn{2}{|c|}{ Excluding $\mathrm{L}$} \\
\hline & Test Statistic & Prob. Value & Test Statistic & Prob. Value & Test Statistic & Prob. Value \\
\hline MOR0 & 3.559 & $0.313 \mathrm{E}+00$ & 5.199 & $0.267 \mathrm{E}+00$ & 31.860 & $0.987 \mathrm{E}-04$ \\
\hline MOR1 & 8.568 & $0.356 \mathrm{E}-01$ & 30.727 & $0.348 \mathrm{E}-05$ & 17.994 & $0.213 \mathrm{E}-01$ \\
\hline \multirow[t]{2}{*}{ MOR2 } & 11.926 & $0.764 \mathrm{E}-02$ & 7.847 & $0.973 \mathrm{E}-01$ & 15.350 & $0.527 \mathrm{E}-01$ \\
\hline & \multicolumn{2}{|c|}{ Excluding $\mathrm{C}$} & \multicolumn{2}{|c|}{ Excluding $\mathrm{V}$} & \multicolumn{2}{|c|}{ Excluding $\mathrm{T}$} \\
\hline Data & Test Statistic & Prob. Value & Test Statistic & Prob. Value & Test Statistic & Prob. Value \\
\hline MOR0 & 4.040 & $0.401 \mathrm{E}+00$ & 20.569 & $0.385 \mathrm{E}-03$ & 10.684 & $0.304 \mathrm{E}-01$ \\
\hline MOR1 & 3.813 & $0.432 \mathrm{E}+00$ & 7.237 & $0.124 \mathrm{E}+00$ & 20.356 & $0.425 \mathrm{E}-03$ \\
\hline MOR2 & 10.941 & $0.272 \mathrm{E}-01$ & 7.388 & $0.117 \mathrm{E}+00$ & 12.234 & $0.157 \mathrm{E}-01$ \\
\hline
\end{tabular}


Table 6: Model Specification Tests Based on GMM Estimation.

$F A C$ System

REN0, REN1 and REN2 Households.

Tests Based on Estimation with Four-Region Data, 1980-1992.

\begin{tabular}{|c|c|c|c|c|c|c|}
\hline \multirow[b]{2}{*}{ Data } & \multicolumn{2}{|c|}{ Excluding Q } & \multicolumn{2}{|c|}{ Excluding $\mathrm{A}$} & \multicolumn{2}{|c|}{ Excluding L } \\
\hline & Test Statistic & Prob. Value & Test Statistic & Prob. Value & Test Statistic & Prob. Value \\
\hline REN0 & 13.596 & $0.351 \mathrm{E}-02$ & 3.441 & $0.487 \mathrm{E}+00$ & 13.981 & $0.823 \mathrm{E}-01$ \\
\hline REN1 & 18.318 & $0.378 \mathrm{E}-0.3$ & 11.310 & $0.233 \mathrm{E}-01$ & 18.800 & $0.160 \mathrm{E}-01$ \\
\hline \multirow[t]{2}{*}{ REN2 } & 8.044 & $0.451 \mathrm{E}-01$ & 31.298 & $0.266 \mathrm{E}-05$ & 15.506 & $0.500 \mathrm{E}-01$ \\
\hline & \multicolumn{2}{|c|}{ Excluding $\mathrm{C}$} & \multicolumn{2}{|c|}{ Excluding $\mathrm{V}$} & \multicolumn{2}{|c|}{ Excluding $\mathrm{T}$} \\
\hline Data & Test Statistic & Prob. Value & Test Statistic & Prob. Value & Test Statistic & Prob. Value \\
\hline REN0 & 1.298 & $0.862 \mathrm{E}+00$ & 23.090 & $0.121 \mathrm{E}-03$ & 7.303 & $0.121 \mathrm{E}+00$ \\
\hline REN1 & 5.062 & $0.281 \mathrm{E}+00$ & 2.703 & $0.609 \mathrm{E}+00$ & 3.534 & $0.473 \mathrm{E}+00$ \\
\hline REN2 & 12.109 & $0.166 \mathrm{E}-01$ & 32.249 & $0.170 \mathrm{E}-05$ & 3.485 & $0.480 \mathrm{E}+00$ \\
\hline
\end{tabular}

Tests Based on Estimation with Four-Region Data, 1980-1985.

\begin{tabular}{|c|c|c|c|c|c|c|}
\hline \multirow[b]{2}{*}{ Data } & \multicolumn{2}{|c|}{ Excluding Q } & \multicolumn{2}{|c|}{ Excluding $\mathrm{A}$} & \multicolumn{2}{|c|}{ Excluding L } \\
\hline & Test Statistic & Prob. Value & Test Statistic & Prob. Value & Test Statistic & Prob. Value \\
\hline REN0 & 1.106 & $0.776 \mathrm{E}+00$ & 15.400 & $0.394 \mathrm{E}-02$ & 10.818 & $0.212 \mathrm{E}+00$ \\
\hline REN1 & 5.058 & $0.168 \mathrm{E}+00$ & 30.230 & $0.439 \mathrm{E}-05$ & 9.637 & $0.291 \mathrm{E}+00$ \\
\hline \multirow[t]{2}{*}{ REN2 } & 14.996 & $0.182 \mathrm{E}-02$ & 5.052 & $0.282 \mathrm{E}+00$ & 46.558 & $0.186 \mathrm{E}-06$ \\
\hline & \multicolumn{2}{|c|}{ Excluding $\mathrm{C}$} & \multicolumn{2}{|c|}{ Excluding $\mathrm{V}$} & \multicolumn{2}{|c|}{ Excluding $\mathrm{T}$} \\
\hline Data & Test Statistic & Prob. Value & Test Statistic & Prob. Value & Test Statistic & Prob. Value \\
\hline REN0 & 8.370 & $0.789 \mathrm{E}-01$ & 14.202 & $0.668 \mathrm{E}-02$ & 4.670 & $0.323 \mathrm{E}+00$ \\
\hline REN1 & 4.558 & $0.336 \mathrm{E}+00$ & 4.600 & $0.331 \mathrm{E}+00$ & 7.729 & $0.102 \mathrm{E}+00$ \\
\hline REN2 & 5.819 & $0.213 \mathrm{E}+00$ & 43.253 & $0.917 \mathrm{E}-08$ & 4.915 & $0.296 \mathrm{E}+00$ \\
\hline
\end{tabular}

Tests Based on Estimation with Three-Region Data, 1980-1992.

\begin{tabular}{|c|c|c|c|c|c|c|}
\hline \multirow[b]{2}{*}{ Data } & \multicolumn{2}{|c|}{ Excluding $\mathrm{Q}$} & \multicolumn{2}{|c|}{ Excluding $\mathrm{A}$} & \multicolumn{2}{|c|}{ Excluding $\mathrm{L}$} \\
\hline & Test Statistic & Prob. Value & Test Statistic & Prob. Value & Test Statistic & Prob. Value \\
\hline REN0 & 8.919 & $0.304 \mathrm{E}-01$ & 16.106 & $0.288 \mathrm{E}-02$ & 14.351 & $0.731 \mathrm{E}-01$ \\
\hline REN1 & 2.274 & $0.518 \mathrm{E}+00$ & 16.337 & $0.260 \mathrm{E}-02$ & 14.434 & $0.711 \mathrm{E}-01$ \\
\hline \multirow[t]{2}{*}{ REN2 } & 2.376 & $0.498 \mathrm{E}+00$ & 12.321 & $0.151 \mathrm{E}-01$ & 46.041 & $0.233 \mathrm{E}-06$ \\
\hline & \multicolumn{2}{|c|}{ Excluding $\mathrm{C}$} & \multicolumn{2}{|c|}{ Excluding $\mathrm{V}$} & \multicolumn{2}{|c|}{ Excluding $\mathrm{T}$} \\
\hline Data & Test Statistic & Prob. Value & Test Statistic & Prob. Value & Test Statistic & Prob. Value \\
\hline REN0 & 6.335 & $0.175 \mathrm{E}+00$ & 1.968 & $0.742 \mathrm{E}+00$ & 4.376 & $0.357 \mathrm{E}+00$ \\
\hline REN1 & 4.266 & $0.371 \mathrm{E}+00$ & 1.214 & $0.876 \mathrm{E}+00$ & 1.464 & $0.833 \mathrm{E}+00$ \\
\hline REN2 & 12.927 & $0.116 \mathrm{E}-01$ & 3.694 & $0.449 \mathrm{E}+00$ & 7.792 & $0.995 \mathrm{E}-01$ \\
\hline
\end{tabular}


Table 7: Model Specification Tests Based on GMM Estimation.

GOP System

MOR0, MOR1 and MOR2 Households.

Tests Based on Estimation with Four-Region Data, 1980-1992.

\begin{tabular}{|c|c|c|c|c|c|c|}
\hline \multirow[b]{2}{*}{ Data } & \multicolumn{2}{|c|}{ Excluding $\mathrm{Q}$} & \multicolumn{2}{|c|}{ Excluding $\mathrm{A}$} & \multicolumn{2}{|c|}{ Excluding L } \\
\hline & Test Statistic & Prob. Value & Test Statistic & Prob. Value & Test Statistic & Prob. Value \\
\hline MOR0 & 28.615 & $0.270 \mathrm{E}-05$ & 10.379 & $0.345 \mathrm{E}-01$ & 13.395 & $0.990 \mathrm{E}-01$ \\
\hline MOR1 & 3.109 & $0.375 \mathrm{E}+00$ & 11.344 & $0.230 \mathrm{E}-01$ & 5.334 & $0.721 \mathrm{E}+00$ \\
\hline \multirow[t]{2}{*}{ MOR2 } & 0.902 & $0.825 \mathrm{E}+00$ & 8.301 & $0.811 \mathrm{E}-01$ & 7.241 & $0.511 \mathrm{E}+00$ \\
\hline & \multicolumn{2}{|c|}{ Excluding $\mathrm{C}$} & \multicolumn{2}{|c|}{ Excluding $\mathrm{V}$} & \multicolumn{2}{|c|}{ Excluding $\mathrm{T}$} \\
\hline Data & Test Statistic & Prob. Value & Test Statistic & Prob. Value & Test Statistic & Prob. Value \\
\hline MOR0 & 12.050 & $0.170 \mathrm{E}-01$ & 12.699 & $0.128 \mathrm{E}-01$ & 9.190 & $0.565 \mathrm{E}-01$ \\
\hline MOR1 & 6.797 & $0.147 \mathrm{E}+00$ & 3.198 & $0.525 \mathrm{E}+00$ & 7.298 & $0.121 \mathrm{E}+00$ \\
\hline MOR2 & 13.930 & $0.752 \mathrm{E}-02$ & 6.086 & $0.193 \mathrm{E}+00$ & 10.630 & $0.310 \mathrm{E}-01$ \\
\hline
\end{tabular}

Tests Based on Estimation with Four-Region Data, 1980-1985.

\begin{tabular}{|c|c|c|c|c|c|c|}
\hline \multirow[b]{2}{*}{ Data } & \multicolumn{2}{|c|}{ Excluding Q } & \multicolumn{2}{|c|}{ Excluding A } & \multicolumn{2}{|c|}{ Excluding L } \\
\hline & Test Statistic & Prob. Value & Test Statistic & Prob. Value & Test Statistic & Prob. Value \\
\hline MOR0 & 10.370 & $0.157 \mathrm{E}-01$ & 7.846 & $0.974 \mathrm{E}-01$ & 8.466 & $0.389 \mathrm{E}+00$ \\
\hline MOR1 & 10.673 & $0.136 \mathrm{E}-01$ & 11.815 & $0.188 \mathrm{E}-01$ & 7.131 & $0.523 \mathrm{E}+00$ \\
\hline MOR2 & 3.327 & $0.344 \mathrm{E}+00$ & 21.065 & $0.307 \mathrm{E}-03$ & 20.248 & $0.944 \mathrm{E}-02$ \\
\hline \multirow[b]{2}{*}{ Data } & \multicolumn{2}{|c|}{ Excluding $\mathrm{C}$} & \multicolumn{2}{|c|}{ Excluding $V$} & \multicolumn{2}{|c|}{ Excluding $T$} \\
\hline & Test Statistic & Prob. Value & Test Statistic & Prob. Value & Test Statistic & Prob. Value \\
\hline MOR0 & 41.102 & $0.256 \mathrm{E}-07$ & 26.893 & $0.209 \mathrm{E}-04$ & 11.683 & $0.199 \mathrm{E}-01$ \\
\hline MOR1 & 5.092 & $0.278 \mathrm{E}+00$ & 11.453 & $0.219 \mathrm{E}-01$ & 3.122 & $0.538 \mathrm{E}+00$ \\
\hline MOR2 & 6.362 & $0.174 \mathrm{E}+00$ & 7.059 & $0.133 \mathrm{E}+00$ & 9.704 & $0.457 \mathrm{E}-01$ \\
\hline
\end{tabular}

Tests Based on Estimation with Three-Region Data, 1980-1992.

\begin{tabular}{|c|c|c|c|c|c|c|}
\hline \multirow[b]{2}{*}{ Data } & \multicolumn{2}{|c|}{ Excluding Q } & \multicolumn{2}{|c|}{ Excluding A } & \multicolumn{2}{|c|}{ Excluding L } \\
\hline & Test Statistic & Prob. Value & Test Statistic & Prob. Value & Test Statistic & Prob. Value \\
\hline MOR0 & 2.299 & $0.513 \mathrm{E}+00$ & 3.655 & $0.455 \mathrm{E}+00$ & 12.785 & $0.119 \mathrm{E}+00$ \\
\hline MOR1 & 1.419 & $0.701 \mathrm{E}+00$ & 4.926 & $0.295 \mathrm{E}+00$ & 6.467 & $0.595 \mathrm{E}+00$ \\
\hline MOR2 & 0.629 & $0.890 \mathrm{E}+00$ & 6.188 & $0.186 \mathrm{E}+00$ & 11.753 & $0.163 \mathrm{E}+00$ \\
\hline \multirow[b]{2}{*}{ Data } & \multicolumn{2}{|c|}{ Excluding C } & \multicolumn{2}{|c|}{ Excluding V } & \multicolumn{2}{|c|}{ Excluding $\mathrm{T}$} \\
\hline & Test Statistic & Prob. Value & Test Statistic & Prob. Value & Test Statistic & Prob. Value \\
\hline MOR0 & 5.141 & $0.273 \mathrm{E}+00$ & 9.696 & $0.459 \mathrm{E}-01$ & 9.676 & $0.463 \mathrm{E}-01$ \\
\hline MOR1 & 5.283 & $0.259 \mathrm{E}+00$ & 5.200 & $0.267 \mathrm{E}+00$ & 1.377 & $0.848 \mathrm{E}+00$ \\
\hline MOR2 & 22.734 & $0.143 \mathrm{E}-03$ & 4.058 & $0.398 \mathrm{E}+00$ & 10.609 & $0.313 \mathrm{E}-01$ \\
\hline
\end{tabular}


Table 8: Model Specification Tests Based on GMM Estimation.

GOP System

REN0, REN1 and REN2 Households.

Tests Based on Estimation with Four-Region Data, 1980-1992.

\begin{tabular}{|c|c|c|c|c|c|c|}
\hline \multirow[b]{2}{*}{ Data } & \multicolumn{2}{|c|}{ Excluding Q } & \multicolumn{2}{|c|}{ Excluding A } & \multicolumn{2}{|c|}{ Excluding L } \\
\hline & Test Statistic & Prob. Value & Test Statistic & Prob. Value & Test Statistic & Prob. Value \\
\hline REN0 & 4.718 & $0.194 \mathrm{E}+00$ & 4.518 & $0.340 \mathrm{E}+00$ & 12.219 & $0.142 \mathrm{E}+00$ \\
\hline REN1 & 0.428 & $0.934 \mathrm{E}+00$ & 11.943 & $0.178 \mathrm{E}-01$ & 13.299 & $0.102 \mathrm{E}+00$ \\
\hline \multirow[t]{2}{*}{ REN2 } & 2.738 & $0.434 \mathrm{E}+00$ & 7.785 & $0.998 \mathrm{E}-01$ & 30.696 & $0.159 \mathrm{E}-03$ \\
\hline & \multicolumn{2}{|c|}{ Excluding $\mathrm{C}$} & \multicolumn{2}{|c|}{ Excluding $\mathrm{V}$} & \multicolumn{2}{|c|}{ Excluding $\mathrm{T}$} \\
\hline Data & Test Statistic & Prob. Value & Test Statistic & Prob. Value & Test Statistic & Prob. Value \\
\hline REN0 & 1.391 & $0.846 \mathrm{E}+00$ & 17.278 & $0.171 \mathrm{E}-02$ & 6.195 & $0.185 \mathrm{E}+00$ \\
\hline REN1 & 20.416 & $0.413 \mathrm{E}-03$ & 11.518 & $0.213 \mathrm{E}-01$ & 11.761 & $0.192 \mathrm{E}-01$ \\
\hline REN2 & 3.857 & $0.426 \mathrm{E}+00$ & 15.625 & $0.357 \mathrm{E}-02$ & 10.196 & $0.373 \mathrm{E}-01$ \\
\hline
\end{tabular}

Tests Based on Estimation with Four-Region Data, 1980-1985.

\begin{tabular}{|c|c|c|c|c|c|c|}
\hline \multirow[b]{2}{*}{ Data } & \multicolumn{2}{|c|}{ Excluding Q } & \multicolumn{2}{|c|}{ Excluding A } & \multicolumn{2}{|c|}{ Excluding L } \\
\hline & Test Statistic & Prob. Value & Test Statistic & Prob. Value & Test Statistic & Prob. Value \\
\hline REN0 & 4.634 & $0.201 \mathrm{E}+00$ & 13.053 & $0.110 \mathrm{E}-01$ & 16.617 & $0.344 \mathrm{E}-01$ \\
\hline REN1 & 1.768 & $0.622 \mathrm{E}+00$ & 9.763 & $0.446 \mathrm{E}-01$ & 3.501 & $0.899 \mathrm{E}+00$ \\
\hline REN2 & 2.811 & $0.422 \mathrm{E}+00$ & 10.948 & $0.272 \mathrm{E}-01$ & 32.361 & $0.803 \mathrm{E}-04$ \\
\hline \multirow[b]{2}{*}{ Data } & \multicolumn{2}{|c|}{ Excluding C } & \multicolumn{2}{|c|}{ Excluding V } & \multicolumn{2}{|c|}{ Excluding $\mathrm{T}$} \\
\hline & Test Statistic & Prob. Value & Test Statistic & Prob. Value & Test Statistic & Prob. Value \\
\hline REN0 & 4.340 & $0.362 \mathrm{E}+00$ & 11.672 & $0.200 \mathrm{E}-01$ & 14.682 & $0.541 \mathrm{E}-02$ \\
\hline REN1 & 5.542 & $0.236 \mathrm{E}+00$ & 2.597 & $0.627 \mathrm{E}+00$ & 9.822 & $0.435 \mathrm{E}-01$ \\
\hline REN2 & 12.937 & $0.116 \mathrm{E}-01$ & 4.648 & $0.325 \mathrm{E}+00$ & 16.426 & $0.250 \mathrm{E}-02$ \\
\hline
\end{tabular}

Tests Based on Estimation with Three-Region Data, 1980-1992.

\begin{tabular}{|c|c|c|c|c|c|c|}
\hline \multirow[b]{2}{*}{ Data } & \multicolumn{2}{|c|}{ Excluding Q } & \multicolumn{2}{|c|}{ Excluding A } & \multicolumn{2}{|c|}{ Excluding L } \\
\hline & Test Statistic & Prob. Value & Test Statistic & Prob. Value & Test Statistic & Prob. Value \\
\hline REN0 & 3.788 & $0.285 \mathrm{E}+00$ & 5.026 & $0.285 \mathrm{E}+00$ & 18.091 & $0.206 \mathrm{E}-01$ \\
\hline REN1 & 0.001 & $0.100 \mathrm{E}+01$ & 6.098 & $0.192 \mathrm{E}+00$ & 5.958 & $0.652 \mathrm{E}+00$ \\
\hline REN2 & 6.283 & $0.986 \mathrm{E}-01$ & 10.945 & $0.272 \mathrm{E}-01$ & 12.797 & $0.119 \mathrm{E}+00$ \\
\hline \multirow[b]{2}{*}{ Data } & \multicolumn{2}{|c|}{ Excluding $\mathrm{C}$} & \multicolumn{2}{|c|}{ Excluding V } & \multicolumn{2}{|c|}{ Excluding $\mathrm{T}$} \\
\hline & Test Statistic & Prob. Value & Test Statistic & Prob. Value & Test Statistic & Prob. Value \\
\hline REN0 & 3.982 & $0.408 \mathrm{E}+00$ & 10.327 & $0.353 \mathrm{E}-01$ & 12.672 & $0.130 \mathrm{E}-01$ \\
\hline REN1 & 4.906 & $0.297 \mathrm{E}+00$ & 6.061 & $0.195 \mathrm{E}+00$ & 2.189 & $0.701 \mathrm{E}+00$ \\
\hline REN2 & 1.192 & $0.879 \mathrm{E}+00$ & 50.823 & $0.243 \mathrm{E}-09$ & 4.285 & $0.369 \mathrm{E}+00$ \\
\hline
\end{tabular}


Table 9: Model Specification Tests Based on GMM Estimation.

HES System

MOR0, MOR1 and MOR2 Households.

Tests Based on Estimation with Four-Region Data, 1980-1992.

\begin{tabular}{|c|c|c|c|c|c|c|}
\hline \multirow[b]{2}{*}{ Data } & \multicolumn{2}{|c|}{ Excluding $\mathrm{Q}$} & \multicolumn{2}{|c|}{ Excluding A } & \multicolumn{2}{|c|}{ Excluding L } \\
\hline & Test Statistic & Prob. Value & Test Statistic & Prob. Value & Test Statistic & Prob. Value \\
\hline MOR0 & 1.432 & $0.698 \mathrm{E}+00$ & 2.488 & $0.647 \mathrm{E}+00$ & 3.182 & $0.922 \mathrm{E}+00$ \\
\hline MOR1 & 3.191 & $0.363 \mathrm{E}+00$ & 1.332 & $0.856 \mathrm{E}+00$ & 21.100 & $0.689 \mathrm{E}-02$ \\
\hline \multirow[t]{2}{*}{ MOR2 } & 6.229 & $0.101 \mathrm{E}+00$ & 23.942 & $0.821 \mathrm{E}-04$ & 7.789 & $0.454 \mathrm{E}+00$ \\
\hline & \multicolumn{2}{|c|}{ Excluding $\mathrm{C}$} & \multicolumn{2}{|c|}{ Excluding $\mathrm{V}$} & \multicolumn{2}{|c|}{ Excluding $\mathrm{T}$} \\
\hline Data & Test Statistic & Prob. Value & Test Statistic & Prob. Value & Test Statistic & Prob. Value \\
\hline MOR0 & 8.044 & $0.900 \mathrm{E}-01$ & 3.409 & $0.492 \mathrm{E}+00$ & 4.620 & $0.329 \mathrm{E}+00$ \\
\hline MOR1 & 3.350 & $0.501 \mathrm{E}+00$ & 15.502 & $0.377 \mathrm{E}-02$ & 0.735 & $0.947 \mathrm{E}+00$ \\
\hline MOR2 & 8.557 & $0.732 \mathrm{E}-01$ & 2.108 & $0.716 \mathrm{E}+00$ & 5.558 & $0.235 \mathrm{E}+00$ \\
\hline
\end{tabular}

Tests Based on Estimation with Four-Region Data, 1980-1985.

\begin{tabular}{|c|c|c|c|c|c|c|}
\hline \multirow[b]{2}{*}{ Data } & \multicolumn{2}{|c|}{ Excluding $\mathrm{Q}$} & \multicolumn{2}{|c|}{ Excluding $\mathrm{A}$} & \multicolumn{2}{|c|}{ Excluding L } \\
\hline & Test Statistic & Prob. Value & Test Statistic & Prob. Value & Test Statistic & Prob. Value \\
\hline MOR0 & 1.189 & $0.756 \mathrm{E}+00$ & 6.463 & $0.167 \mathrm{E}+00$ & 2.577 & $0.958 \mathrm{E}+00$ \\
\hline MOR1 & 8.200 & $0.421 \mathrm{E}-01$ & 0.156 & $0.997 \mathrm{E}+00$ & 11.393 & $0.180 \mathrm{E}+00$ \\
\hline \multirow[t]{2}{*}{ MOR2 } & 1.668 & $0.644 \mathrm{E}+00$ & 16.323 & $0.261 \mathrm{E}-02$ & 13.927 & $0.837 \mathrm{E}-01$ \\
\hline & \multicolumn{2}{|c|}{ Excluding $\mathrm{C}$} & \multicolumn{2}{|c|}{ Excluding $\mathrm{V}$} & \multicolumn{2}{|c|}{ Excluding $\mathrm{T}$} \\
\hline Data & Test Statistic & Prob. Value & Test Statistic & Prob. Value & Test Statistic & Prob. Value \\
\hline MOR0 & 0.758 & $0.944 \mathrm{E}+00$ & 2.208 & $0.698 \mathrm{E}+00$ & 1.083 & $0.897 \mathrm{E}+00$ \\
\hline MOR1 & 23.221 & $0.114 \mathrm{E}-03$ & 6.378 & $0.173 \mathrm{E}+00$ & 13.209 & $0.103 \mathrm{E}-01$ \\
\hline MOR2 & 9.780 & $0.443 \mathrm{E}-01$ & 5.101 & $0.277 \mathrm{E}+00$ & 4.657 & $0.324 \mathrm{E}+00$ \\
\hline
\end{tabular}

Tests Based on Estimation with Three-Region Data, 1980-1992.

\begin{tabular}{|c|c|c|c|c|c|c|}
\hline \multirow[b]{2}{*}{ Data } & \multicolumn{2}{|c|}{ Excluding $\mathrm{Q}$} & \multicolumn{2}{|c|}{ Excluding $\mathrm{A}$} & \multicolumn{2}{|c|}{ Excluding L } \\
\hline & Test Statistic & Prob. Value & Test Statistic & Prob. Value & Test Statistic & Prob. Value \\
\hline MOR0 & 3.155 & $0.368 \mathrm{E}+00$ & 10.949 & $0.271 \mathrm{E}-01$ & 6.304 & $0.613 \mathrm{E}+00$ \\
\hline MOR1 & 4.165 & $0.244 \mathrm{E}+00$ & 3.384 & $0.496 \mathrm{E}+00$ & 34.352 & $0.351 \mathrm{E}-04$ \\
\hline \multirow[t]{2}{*}{ MOR2 } & 6.367 & $0.951 \mathrm{E}-01$ & 25.780 & $0.350 \mathrm{E}-04$ & 16.423 & $0.367 \mathrm{E}-01$ \\
\hline & \multicolumn{2}{|c|}{ Excluding $\mathrm{C}$} & \multicolumn{2}{|c|}{ Excluding $\mathrm{V}$} & \multicolumn{2}{|c|}{ Excluding $\mathrm{T}$} \\
\hline Data & Test Statistic & Prob. Value & Test Statistic & Prob. Value & Test Statistic & Prob. Value \\
\hline MOR0 & 8.797 & $0.664 \mathrm{E}-01$ & 8.961 & $0.621 \mathrm{E}-01$ & 7.187 & $0.126 \mathrm{E}+00$ \\
\hline MOR1 & 6.066 & $0.194 \mathrm{E}+00$ & 14.051 & $0.713 \mathrm{E}-02$ & 2.986 & $0.560 \mathrm{E}+00$ \\
\hline MOR2 & 2.263 & $0.688 \mathrm{E}+00$ & 8.456 & $0.762 \mathrm{E}-01$ & 2.751 & $0.600 \mathrm{E}+00$ \\
\hline
\end{tabular}


Table 10: Model Specification Tests Based on GMM Estimation.

HES System

REN0, REN1 and REN2 Households.

Tests Based on Estimation with Four-Region Data, 1980-1992.

\begin{tabular}{|c|c|c|c|c|c|c|}
\hline \multirow[b]{2}{*}{ Data } & \multicolumn{2}{|c|}{ Excluding Q } & \multicolumn{2}{|c|}{ Excluding A } & \multicolumn{2}{|c|}{ Excluding L } \\
\hline & Test Statistic & Prob. Value & Test Statistic & Prob. Value & Test Statistic & Prob. Value \\
\hline REN0 & 1.835 & $0.607 \mathrm{E}+00$ & 3.731 & $0.444 \mathrm{E}+00$ & 3.427 & $0.905 \mathrm{E}+00$ \\
\hline REN1 & 1.653 & $0.647 \mathrm{E}+00$ & 9.194 & $0.564 \mathrm{E}-01$ & 16.031 & $0.419 \mathrm{E}-01$ \\
\hline \multirow[t]{2}{*}{ REN2 } & 1.508 & $0.680 \mathrm{E}+00$ & 46.956 & $0.156 \mathrm{E}-08$ & 23.507 & $0.277 \mathrm{E}-02$ \\
\hline & \multicolumn{2}{|c|}{ Excluding $\mathrm{C}$} & \multicolumn{2}{|c|}{ Excluding $\mathrm{V}$} & \multicolumn{2}{|c|}{ Excluding $\mathrm{T}$} \\
\hline Data & Test Statistic & Prob. Value & Test Statistic & Prob. Value & Test Statistic & Prob. Value \\
\hline REN0 & 0.290 & $0.990 \mathrm{E}+00$ & 3.803 & $0.433 \mathrm{E}+00$ & 2.893 & $0.576 \mathrm{E}+00$ \\
\hline REN1 & 7.870 & $0.964 \mathrm{E}-01$ & 10.015 & $0.402 \mathrm{E}-01$ & 4.920 & $0.296 \mathrm{E}+00$ \\
\hline REN2 & 9.841 & $0.432 \mathrm{E}-01$ & 0.023 & $0.100 \mathrm{E}+01$ & 0.822 & $0.935 \mathrm{E}+00$ \\
\hline
\end{tabular}

Tests Based on Estimation with Four-Region Data, 1980-1985.

\begin{tabular}{|c|c|c|c|c|c|c|}
\hline \multirow[b]{2}{*}{ Data } & \multicolumn{2}{|c|}{ Excluding Q } & \multicolumn{2}{|c|}{ Excluding $\mathrm{A}$} & \multicolumn{2}{|c|}{ Excluding L } \\
\hline & Test Statistic & Prob. Value & Test Statistic & Prob. Value & Test Statistic & Prob. Value \\
\hline REN0 & 1.720 & $0.633 \mathrm{E}+00$ & 5.980 & $0.201 \mathrm{E}+00$ & 16.032 & $0.419 \mathrm{E}-01$ \\
\hline REN1 & 1.422 & $0.700 \mathrm{E}+00$ & 10.604 & $0.314 \mathrm{E}-01$ & 39.487 & $0.399 \mathrm{E}-05$ \\
\hline \multirow[t]{2}{*}{ REN2 } & 7.282 & $0.634 \mathrm{E}-01$ & 9.023 & $0.605 \mathrm{E}-01$ & 11.994 & $0.151 \mathrm{E}+00$ \\
\hline & \multicolumn{2}{|c|}{ Excluding $\mathrm{C}$} & \multicolumn{2}{|c|}{ Excluding $\mathrm{V}$} & \multicolumn{2}{|c|}{ Excluding $\mathrm{T}$} \\
\hline Data & Test Statistic & Prob. Value & Test Statistic & Prob. Value & Test Statistic & Prob. Value \\
\hline REN0 & 5.833 & $0.212 \mathrm{E}+00$ & 19.178 & $0.725 \mathrm{E}-03$ & 2.824 & $0.588 \mathrm{E}+00$ \\
\hline REN1 & 9.266 & $0.548 \mathrm{E}-01$ & 3.324 & $0.505 \mathrm{E}+00$ & 5.328 & $0.255 \mathrm{E}+00$ \\
\hline REN2 & 14.219 & $0.663 \mathrm{E}-02$ & 11.102 & $0.254 \mathrm{E}-01$ & 3.554 & $0.470 \mathrm{E}+00$ \\
\hline
\end{tabular}

Tests Based on Estimation with Three-Region Data, 1980-1992.

\begin{tabular}{|c|c|c|c|c|c|c|}
\hline \multirow[b]{2}{*}{ Data } & \multicolumn{2}{|c|}{ Excluding $\mathrm{Q}$} & \multicolumn{2}{|c|}{ Excluding $\mathrm{A}$} & \multicolumn{2}{|c|}{ Excluding $\mathrm{L}$} \\
\hline & Test Statistic & Prob. Value & Test Statistic & Prob. Value & Test Statistic & Prob. Value \\
\hline REN0 & 1.200 & $0.753 \mathrm{E}+00$ & 3.422 & $0.490 \mathrm{E}+00$ & 9.420 & $0.308 \mathrm{E}+00$ \\
\hline REN1 & 1.512 & $0.679 \mathrm{E}+00$ & 5.037 & $0.284 \mathrm{E}+00$ & 27.195 & $0.654 \mathrm{E}-03$ \\
\hline \multirow[t]{2}{*}{ REN2 } & 1.328 & $0.722 \mathrm{E}+00$ & 14.197 & $0.669 \mathrm{E}-02$ & 18.988 & $0.149 \mathrm{E}-01$ \\
\hline & \multicolumn{2}{|c|}{ Excluding $\mathrm{C}$} & \multicolumn{2}{|c|}{ Excluding $\mathrm{V}$} & \multicolumn{2}{|c|}{ Excluding $\mathrm{T}$} \\
\hline Data & Test Statistic & Prob. Value & Test Statistic & Prob. Value & Test Statistic & Prob. Value \\
\hline REN0 & 2.813 & $0.590 \mathrm{E}+00$ & 1.234 & $0.872 \mathrm{E}+00$ & 6.501 & $0.165 \mathrm{E}+00$ \\
\hline REN1 & 3.826 & $0.430 \mathrm{E}+00$ & 11.122 & $0.252 \mathrm{E}-01$ & 7.004 & $0.136 \mathrm{E}+00$ \\
\hline REN2 & 6.607 & $0.158 \mathrm{E}+00$ & 4.740 & $0.315 \mathrm{E}+00$ & 3.466 & $0.483 \mathrm{E}+00$ \\
\hline
\end{tabular}


Table 11: FACGOPHES System Elasticity Estimates, MOR0 and REN0 Households. Estimates Based on Four-Region Data, 1980-1985.

\begin{tabular}{|c|c|c|c|c|c|c|c|c|c|}
\hline \multirow[b]{2}{*}{ Equation } & \multicolumn{9}{|c|}{ 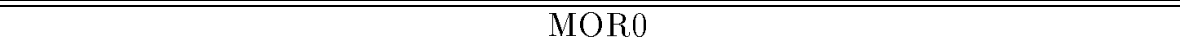 } \\
\hline & $\mathrm{F}$ & A & $\mathrm{C}$ & $\mathrm{G}$ & $\mathrm{O}$ & $\mathrm{P}$ & $\mathrm{H}$ & $\mathrm{E}$ & $\mathrm{S}$ \\
\hline \multirow[t]{2}{*}{$\mathrm{F}$} & -1.234 & 0.288 & 0.107 & 0.382 & -0.126 & -0.131 & -0.084 & 0.000 & 0.021 \\
\hline & 4.689 & 2.421 & 1.246 & 3.142 & 0.941 & 2.151 & 1.326 & 0.005 & 0.206 \\
\hline \multirow[t]{2}{*}{ A } & 1.746 & -1.927 & -0.884 & -0.922 & -0.799 & -0.129 & 1.454 & 0.022 & 0.177 \\
\hline & 2.257 & 3.185 & 3.138 & 2.419 & 1.504 & 0.610 & 4.223 & 0.075 & 0.702 \\
\hline \multirow[t]{2}{*}{ C } & 0.061 & -0.427 & -1.255 & -0.204 & 0.134 & 0.371 & -0.265 & -0.100 & 0.154 \\
\hline & 0.243 & 3.337 & 9.297 & 1.372 & 0.853 & 4.437 & 3.057 & 0.985 & 0.670 \\
\hline \multirow[t]{2}{*}{$\mathrm{G}$} & 1.225 & -0.305 & -0.013 & -0.675 & -0.073 & 0.067 & -0.072 & -0.305 & -0.068 \\
\hline & 3.863 & 1.963 & 0.096 & 2.622 & 0.339 & 0.596 & 0.759 & 2.321 & 0.203 \\
\hline \multirow[t]{2}{*}{$\mathrm{O}$} & -0.455 & -0.309 & 0.163 & -0.190 & -0.862 & 0.131 & 0.225 & 0.440 & -0.255 \\
\hline & 1.320 & 1.470 & 1.182 & 0.893 & 2.386 & 1.244 & 1.327 & 3.068 & 2.197 \\
\hline \multirow[t]{2}{*}{$\mathrm{P}$} & -1.837 & -0.280 & 0.841 & -0.184 & 0.175 & -1.768 & 0.228 & -0.306 & 0.235 \\
\hline & 0.645 & 0.244 & 0.639 & 0.158 & 0.212 & 2.013 & 0.545 & 1.049 & 0.275 \\
\hline \multirow[t]{2}{*}{$\mathrm{H}$} & -1.362 & 2.457 & -1.065 & -0.530 & 0.871 & 0.341 & -1.761 & -0.194 & -0.691 \\
\hline & 0.425 & 1.777 & 0.693 & 0.365 & 0.693 & 1.073 & 1.880 & 0.530 & 1.123 \\
\hline \multirow[t]{2}{*}{$\mathrm{E}$} & 0.020 & 0.052 & -0.053 & -0.599 & 0.855 & -0.077 & -0.042 & -1.032 & 0.176 \\
\hline & 0.011 & 0.070 & 0.057 & 0.759 & 1.465 & 0.381 & 0.147 & 2.751 & 0.866 \\
\hline \multirow[t]{2}{*}{$\mathrm{S}$} & 0.133 & 0.332 & 0.665 & -0.431 & -1.209 & 0.419 & -0.720 & 0.423 & -0.774 \\
\hline & 0.257 & 0.781 & 2.573 & 1.300 & 2.397 & 2.428 & 1.636 & 2.034 & 1.991 \\
\hline \multirow[t]{2}{*}{$\mathrm{Y}$} & 0.776 & 1.262 & 1.529 & 0.221 & 1.112 & 2.895 & 1.932 & 0.700 & 1.162 \\
\hline & 17.702 & 8.002 & 17.781 & 2.423 & 11.454 & 13.029 & 10.628 & 5.392 & 9.340 \\
\hline \multirow[b]{2}{*}{ Equation } & & & & & REN0 & & & & \\
\hline & $\mathrm{F}$ & A & $\mathrm{C}$ & $\mathrm{G}$ & $\mathrm{O}$ & $\mathrm{P}$ & $\mathrm{H}$ & $\mathrm{E}$ & $\mathrm{S}$ \\
\hline \multirow[t]{2}{*}{$\mathrm{F}$} & -0.601 & -0.156 & 0.100 & 0.134 & 0.216 & -0.134 & -0.212 & 0.009 & -0.173 \\
\hline & 1.384 & 0.775 & 0.599 & 0.727 & 0.821 & 1.203 & 1.702 & 0.062 & 1.453 \\
\hline \multirow[t]{2}{*}{ A } & -1.264 & -0.359 & -0.820 & 0.041 & -1.055 & -0.021 & 1.355 & 0.771 & -0.229 \\
\hline & 1.040 & 0.290 & 1.529 & 0.063 & 0.956 & 0.056 & 2.037 & 1.494 & 0.365 \\
\hline \multirow[t]{2}{*}{ C } & 0.206 & -0.421 & -1.190 & -0.698 & 0.304 & 0.542 & -0.037 & -0.434 & 0.553 \\
\hline & 0.390 & 1.461 & 3.042 & 2.290 & 0.775 & 2.827 & 0.187 & 1.702 & 2.951 \\
\hline \multirow[t]{2}{*}{$\mathrm{G}$} & 0.423 & 0.079 & -0.558 & -0.034 & -1.077 & -0.028 & 0.088 & -0.047 & 0.436 \\
\hline & 0.793 & 0.248 & 2.036 & 0.077 & 2.403 & 0.144 & 0.418 & 0.190 & 2.116 \\
\hline \multirow[t]{2}{*}{$\mathrm{O}$} & 0.630 & -0.407 & 0.303 & -0.997 & -0.082 & 0.061 & 0.461 & -0.088 & -0.583 \\
\hline & 0.916 & 0.843 & 0.948 & 2.465 & 0.098 & 0.269 & 1.286 & 0.292 & 1.860 \\
\hline \multirow[t]{2}{*}{$\mathrm{P}$} & -1.894 & -0.089 & 1.490 & -0.313 & -0.009 & -1.338 & -0.146 & 0.085 & -0.064 \\
\hline & 0.921 & 0.131 & 1.602 & 0.263 & 0.010 & 1.271 & 0.197 & 0.064 & 0.140 \\
\hline \multirow[t]{2}{*}{$\mathrm{H}$} & -4.447 & 3.811 & -0.354 & 0.272 & 2.713 & -0.269 & -3.197 & -0.391 & -0.708 \\
\hline & 1.430 & 1.979 & 0.244 & 0.153 & 1.165 & 0.225 & 1.120 & 0.217 & 0.457 \\
\hline \multirow[t]{2}{*}{$\mathrm{E}$} & -0.041 & 0.758 & -0.733 & -0.140 & -0.239 & 0.100 & -0.093 & -0.613 & -0.051 \\
\hline & 0.020 & 1.312 & 0.779 & 0.113 & 0.301 & 0.119 & 0.127 & 0.218 & 0.114 \\
\hline \multirow[t]{2}{*}{ S } & -2.462 & -0.460 & 2.150 & 1.859 & -2.871 & -0.035 & -0.488 & -0.128 & 1.197 \\
\hline & 1.631 & 0.347 & 2.947 & 2.085 & 1.901 & 0.072 & 0.446 & 0.199 & 0.974 \\
\hline $\mathrm{Y}$ & 0.818 & 1.581 & 1.175 & 0.719 & 0.702 & 2.278 & 2.570 & 1.052 & 1.238 \\
\hline & 6.419 & 4.039 & 4.308 & 2.860 & 2.808 & 4.076 & 3.744 & 2.687 & 2.457 \\
\hline
\end{tabular}


Table 12: FACGOPHES System Elasticity Estimates, MOR1 and REN1 Households. Estimates Based on Four-Region Data, 1980-1985.

\begin{tabular}{|c|c|c|c|c|c|c|c|c|c|}
\hline \multirow[b]{2}{*}{ Equation } & \multicolumn{9}{|c|}{ "MOR1 } \\
\hline & $\mathrm{F}$ & A & $\mathrm{C}$ & G & $\mathrm{O}$ & $\mathrm{P}$ & $\mathrm{H}$ & $\mathrm{E}$ & $\mathrm{S}$ \\
\hline \multirow[t]{2}{*}{ F } & -0.076 & -0.452 & -0.175 & 0.102 & -0.192 & -0.096 & 0.187 & -0.019 & -0.125 \\
\hline & 0.553 & 7.318 & 3.309 & 1.736 & 1.647 & 2.967 & 2.052 & 0.342 & 3.806 \\
\hline \multirow[t]{2}{*}{ A } & -3.752 & 2.471 & 0.122 & -0.428 & -1.169 & 0.512 & 2.141 & -0.651 & -0.269 \\
\hline & 7.570 & 5.394 & 0.654 & 1.579 & 2.223 & 3.406 & 4.560 & 2.978 & 1.263 \\
\hline \multirow[t]{2}{*}{$\mathrm{C}$} & -0.902 & 0.019 & -0.965 & -0.106 & 0.459 & 0.130 & -0.184 & -0.233 & -0.087 \\
\hline & 5.578 & 0.277 & 6.618 & 1.053 & 3.154 & 2.317 & 1.404 & 2.748 & 1.782 \\
\hline \multirow[t]{2}{*}{ G } & 0.355 & -0.148 & 0.034 & -0.243 & -0.161 & -0.010 & -0.432 & -0.265 & 0.161 \\
\hline & 1.935 & 1.491 & 0.331 & 1.482 & 0.914 & 0.152 & 2.637 & 2.575 & 2.999 \\
\hline \multirow[t]{2}{*}{$\mathrm{O}$} & -0.608 & -0.400 & 0.546 & -0.191 & -0.398 & 0.149 & -0.451 & 0.271 & 0.085 \\
\hline & 1.816 & 2.206 & 4.010 & 1.175 & 0.895 & 1.446 & 1.500 & 1.922 & 0.730 \\
\hline \multirow[t]{2}{*}{$\mathrm{P}$} & -1.188 & 0.695 & 0.510 & -0.083 & 0.559 & -1.457 & -0.612 & 0.384 & -0.029 \\
\hline & 3.139 & 3.357 & 2.130 & 0.358 & 1.390 & 7.259 & 1.816 & 1.724 & 0.239 \\
\hline \multirow[t]{2}{*}{$\mathrm{H}$} & 0.683 & 1.314 & -0.254 & -0.836 & -0.909 & -0.280 & -1.985 & 0.605 & 0.080 \\
\hline & 1.445 & 4.414 & 0.965 & 3.030 & 1.686 & 1.767 & 2.814 & 2.753 & 0.343 \\
\hline \multirow[t]{2}{*}{$\mathrm{E}$} & 0.106 & -0.366 & -0.158 & -0.379 & 0.596 & 0.192 & 0.700 & -1.078 & 0.151 \\
\hline & 0.376 & 2.925 & 1.005 & 2.320 & 2.501 & 1.967 & 3.446 & 5.748 & 2.008 \\
\hline \multirow[t]{2}{*}{ S } & -1.742 & -0.457 & -0.276 & 0.745 & 0.461 & -0.025 & 0.280 & 0.399 & -0.169 \\
\hline & 3.616 & 1.240 & 1.248 & 2.983 & 0.796 & 0.166 & 0.432 & 1.803 & 0.444 \\
\hline \multirow[t]{2}{*}{ Y } & 0.847 & 1.024 & 1.870 & 0.708 & 0.998 & 1.220 & 1.583 & 0.236 & 0.785 \\
\hline & 14.626 & 4.482 & 12.789 & 6.056 & 7.722 & 4.221 & 7.455 & 1.217 & 3.393 \\
\hline \multirow[b]{2}{*}{ Equation } & & & & & REN1 & & & & \\
\hline & $\mathrm{F}$ & A & C & G & $\mathrm{O}$ & $\mathrm{P}$ & $\mathrm{H}$ & $\mathrm{E}$ & $\mathrm{S}$ \\
\hline \multirow[t]{2}{*}{ F } & -1.381 & 0.010 & 0.376 & 0.175 & 0.174 & 0.276 & -0.062 & -0.126 & -0.041 \\
\hline & 3.081 & 0.042 & 1.942 & 0.930 & 0.633 & 2.452 & 0.268 & 0.751 & 0.333 \\
\hline \multirow[t]{2}{*}{ A } & -0.345 & -0.133 & -1.005 & 0.680 & -0.977 & -0.353 & -0.167 & 0.952 & -0.248 \\
\hline & 0.175 & 0.068 & 1.162 & 0.668 & 0.576 & 0.612 & 0.107 & 1.103 & 0.289 \\
\hline \multirow[t]{2}{*}{ C } & 0.980 & -0.511 & -1.379 & -0.368 & 0.137 & -0.322 & -0.277 & 0.196 & -0.357 \\
\hline & 1.290 & 1.223 & 2.420 & 0.861 & 0.263 & 1.357 & 0.620 & 0.529 & 1.408 \\
\hline \multirow[t]{2}{*}{ G } & 0.576 & 0.309 & -0.125 & -0.404 & -0.228 & -0.436 & 0.258 & -0.453 & 0.069 \\
\hline & 1.036 & 0.839 & 0.391 & 0.932 & 0.492 & 2.268 & 0.668 & 1.529 & 0.317 \\
\hline \multirow[t]{2}{*}{$\mathrm{O}$} & 0.321 & -0.404 & 0.199 & -0.398 & -0.985 & 0.423 & -0.147 & -0.347 & 0.061 \\
\hline & 0.332 & 0.554 & 0.430 & 0.724 & 0.795 & 1.359 & 0.175 & 0.785 & 0.153 \\
\hline \multirow[t]{2}{*}{$\mathrm{P}$} & 3.422 & -0.526 & -0.960 & -1.962 & 1.581 & -2.296 & -0.382 & 0.748 & -0.585 \\
\hline & 2.353 & 0.579 & 1.242 & 2.329 & 1.384 & 2.976 & 0.424 & 0.908 & 1.314 \\
\hline \multirow[t]{2}{*}{$\mathrm{H}$} & -1.155 & -0.201 & -0.570 & 0.425 & -0.454 & -0.270 & -0.860 & 0.751 & 0.094 \\
\hline & 0.634 & 0.134 & 0.640 & 0.415 & 0.242 & 0.493 & 0.359 & 0.972 & 0.087 \\
\hline \multirow[t]{2}{*}{$\mathrm{E}$} & -0.904 & 0.617 & 0.317 & -0.902 & -0.515 & 0.293 & 0.540 & -1.167 & 0.469 \\
\hline & 1.042 & 1.120 & 0.650 & 1.736 & 0.793 & 0.889 & 1.057 & 1.722 & 1.886 \\
\hline \multirow[t]{2}{*}{ S } & -0.982 & -0.527 & -1.552 & 0.320 & 0.323 & -0.827 & 0.270 & 1.637 & 0.157 \\
\hline & 0.476 & 0.280 & 1.502 & 0.254 & 0.157 & 1.327 & 0.110 & 1.906 & 0.086 \\
\hline Y & 0.600 & 1.595 & 1.902 & 0.433 & 1.277 & 0.961 & 2.240 & 1.251 & 1.182 \\
\hline & 5.888 & 3.334 & 8.008 & 2.208 & 4.961 & 1.544 & 5.346 & 3.368 & 2.357 \\
\hline
\end{tabular}


Table 13: FACGOPHES System Elasticity Estimates, MOR2 and REN2 Households. Estimates Based on Four-Region Data, 1980-1985.

\begin{tabular}{|c|c|c|c|c|c|c|c|c|c|}
\hline & & & & & $\overline{\text { MOR2 }}$ & & & & \\
\hline Equation & $\mathrm{F}$ & A & $\mathrm{C}$ & G & $\mathrm{O}$ & $\mathrm{P}$ & $\mathrm{H}$ & $\mathrm{E}$ & $\mathrm{S}$ \\
\hline F & -0.548 & 0.138 & -0.062 & 0.159 & -0.187 & -0.104 & -0.220 & 0.106 & -0.051 \\
\hline & 3.132 & 1.835 & 1.129 & 2.308 & 2.038 & 2.737 & 2.909 & 1.835 & 1.314 \\
\hline A & 0.943 & -0.756 & -0.240 & -0.199 & -0.423 & -1.014 & -1.643 & 1.722 & 0.180 \\
\hline & 1.387 & 1.509 & 0.946 & 0.558 & 0.720 & 5.140 & 3.212 & 6.413 & 0.666 \\
\hline C & -0.700 & -0.123 & -1.319 & -0.044 & 0.118 & 0.195 & 0.263 & -0.377 & -0.024 \\
\hline & 3.799 & 1.242 & 9.746 & 0.372 & 0.912 & 3.370 & 2.339 & 3.790 & 0.354 \\
\hline G & 0.756 & -0.005 & 0.196 & -0.142 & -0.350 & -0.025 & 0.241 & -0.544 & -0.198 \\
\hline & 3.638 & 0.039 & 1.769 & 0.742 & 1.868 & 0.342 & 1.555 & 4.919 & 2.785 \\
\hline $\mathrm{O}$ & -0.591 & -0.120 & 0.269 & -0.453 & -1.088 & 0.461 & 0.695 & -0.255 & 0.354 \\
\hline & 2.095 & 0.560 & 2.155 & 2.361 & 2.613 & 5.010 & 2.582 & 1.821 & 2.831 \\
\hline $\mathrm{P}$ & -2.005 & -1.738 & 0.828 & -0.364 & 1.965 & -0.893 & -0.961 & 1.367 & -0.188 \\
\hline & 1.602 & 3.270 & 0.991 & 0.353 & 3.353 & 1.400 & 0.989 & 3.882 & 0.611 \\
\hline $\mathrm{H}$ & -2.213 & -1.309 & 0.390 & 0.179 & 1.175 & -0.453 & -0.814 & 0.227 & 0.063 \\
\hline & 1.890 & 2.477 & 0.497 & 0.188 & 1.751 & 1.069 & 0.773 & 0.687 & 0.209 \\
\hline $\mathrm{E}$ & 0.764 & 1.218 & -0.472 & -1.083 & -0.431 & 0.592 & 0.335 & -1.923 & 0.577 \\
\hline & 0.691 & 3.079 & 0.619 & 1.233 & 0.878 & 1.490 & 0.384 & 5.338 & 3.563 \\
\hline$S$ & -0.926 & 0.258 & -0.018 & -1.001 & 1.298 & -0.143 & 0.213 & 1.150 & -2.306 \\
\hline & 1.904 & 0.662 & 0.070 & 3.510 & 2.631 & 0.902 & 0.414 & 4.751 & 5.485 \\
\hline Y & 0.769 & 1.431 & 2.011 & 0.071 & 0.727 & 1.988 & 2.756 & 0.423 & 1.475 \\
\hline & 10.823 & 4.670 & 12.654 & 0.467 & 4.405 & 4.339 & 10.784 & 1.645 & 6.493 \\
\hline & & & & & REN2 & & & & \\
\hline Equation & $\mathrm{F}$ & A & $\mathrm{C}$ & $\mathrm{G}$ & $\mathrm{O}$ & $\mathrm{P}$ & $\mathrm{H}$ & $\mathrm{E}$ & $\mathrm{S}$ \\
\hline $\mathrm{F}$ & -0.032 & -0.463 & -0.493 & 0.463 & 0.251 & -0.338 & -0.474 & 0.209 & 0.127 \\
\hline & 0.054 & 1.432 & 2.296 & 1.915 & 0.794 & 2.109 & 1.676 & 1.042 & 0.252 \\
\hline A & -3.761 & 1.394 & -0.216 & 0.750 & -0.481 & 0.965 & 0.822 & -0.396 & 0.129 \\
\hline & 1.447 & 0.456 & 0.147 & 0.507 & 0.231 & 0.882 & 0.422 & 0.198 & 0.105 \\
\hline C & -2.402 & -0.141 & -0.722 & 0.744 & 0.775 & 0.244 & 0.159 & 0.668 & -0.724 \\
\hline & 2.670 & 0.187 & 1.022 & 1.418 & 1.166 & 0.635 & 0.270 & 1.096 & 0.857 \\
\hline G & 1.535 & 0.327 & 0.681 & -1.810 & -1.749 & 0.379 & 0.768 & -0.928 & -0.403 \\
\hline & 1.704 & 0.480 & 1.460 & 2.566 & 2.745 & 1.078 & 1.299 & 1.827 & 0.822 \\
\hline $\mathrm{O}$ & 0.891 & -0.259 & 0.784 & -1.908 & 1.410 & -0.273 & -0.915 & -0.867 & 0.049 \\
\hline & 0.688 & 0.245 & 1.201 & 2.722 & 0.911 & 0.564 & 0.787 & 1.419 & 0.086 \\
\hline $\mathrm{P}$ & -5.061 & 1.719 & 0.879 & 1.493 & -0.952 & -0.491 & 1.512 & 0.043 & -0.141 \\
\hline & 1.892 & 0.404 & 0.292 & 0.718 & 0.515 & 0.147 & 0.855 & 0.008 & 0.048 \\
\hline $\mathrm{H}$ & -3.758 & 0.732 & 0.282 & 1.502 & -1.669 & 0.763 & -1.912 & 1.625 & 1.158 \\
\hline & 1.554 & 0.210 & 0.115 & 0.868 & 0.772 & 0.527 & 0.667 & 0.376 & 0.463 \\
\hline $\mathrm{E}$ & 1.186 & -0.491 & 1.320 & -2.285 & -1.957 & -0.010 & 1.896 & -1.592 & -0.181 \\
\hline & 0.344 & 0.058 & 0.232 & 0.591 & 0.936 & 0.003 & 0.774 & 0.072 & 0.037 \\
\hline $\mathrm{S}$ & 3.191 & 0.380 & -3.443 & -2.102 & 0.383 & -0.166 & 3.352 & -0.327 & -1.065 \\
\hline & 1.226 & 0.126 & 2.087 & 1.264 & 0.138 & 0.144 & 1.246 & 0.142 & 0.328 \\
\hline Y & 0.751 & 0.793 & 1.399 & 1.201 & 1.088 & 0.999 & 1.278 & 2.114 & -0.203 \\
\hline & 8.292 & 1.644 & 6.921 & 6.920 & 4.555 & 2.194 & 3.403 & 4.710 & 0.381 \\
\hline
\end{tabular}


Table 14: FACGOPHES System Elasticity Estimates, MOR0 and REN0 Households. Estimates Based on Four-Region Data, 1980-1992.

\begin{tabular}{|c|c|c|c|c|c|c|c|c|c|}
\hline \multirow[b]{2}{*}{ Equation } & \multicolumn{9}{|c|}{ 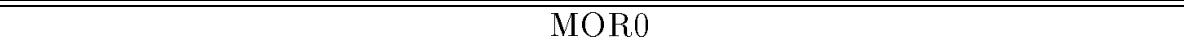 } \\
\hline & $\mathrm{F}$ & A & $\mathrm{C}$ & G & $\mathrm{O}$ & $\mathrm{P}$ & $\mathrm{H}$ & $\mathrm{E}$ & $\mathrm{S}$ \\
\hline \multirow[t]{2}{*}{$\mathrm{F}$} & -0.784 & -0.038 & 0.067 & 0.058 & -0.044 & -0.035 & -0.079 & 0.044 & 0.006 \\
\hline & 5.021 & 0.591 & 1.021 & 1.182 & 0.416 & 0.710 & 1.315 & 0.722 & 0.083 \\
\hline \multirow[t]{2}{*}{ A } & -0.591 & -1.616 & -0.866 & 0.104 & 0.953 & 0.147 & 1.327 & -0.390 & -0.497 \\
\hline & 1.012 & 3.327 & 3.385 & 0.463 & 1.723 & 0.695 & 3.546 & 1.684 & 1.743 \\
\hline \multirow[t]{2}{*}{ C } & -0.052 & -0.279 & -1.239 & -0.079 & 0.249 & -0.017 & -0.102 & 0.004 & 0.033 \\
\hline & 0.273 & 3.395 & 8.755 & 0.857 & 1.642 & 0.202 & 1.280 & 0.039 & 0.213 \\
\hline \multirow[t]{2}{*}{$\mathrm{G}$} & 0.246 & 0.068 & 0.023 & -0.227 & -0.508 & 0.089 & 0.147 & -0.440 & -0.048 \\
\hline & 1.551 & 0.847 & 0.222 & 1.706 & 2.922 & 1.061 & 1.858 & 4.344 & 0.402 \\
\hline \multirow[t]{2}{*}{$\mathrm{O}$} & -0.275 & 0.248 & 0.228 & -0.436 & -1.394 & 0.176 & -0.094 & 0.338 & -0.032 \\
\hline & 1.144 & 1.799 & 1.936 & 3.566 & 4.317 & 1.858 & 0.592 & 2.686 & 0.276 \\
\hline \multirow[t]{2}{*}{$\mathrm{P}$} & -0.862 & 0.120 & -0.185 & 0.104 & 0.563 & -1.242 & -0.247 & -0.508 & 0.023 \\
\hline & 1.797 & 0.525 & 0.602 & 0.352 & 1.388 & 2.615 & 1.134 & 1.552 & 0.058 \\
\hline \multirow[t]{2}{*}{$\mathrm{H}$} & -1.388 & 2.020 & -0.517 & 0.556 & -0.644 & -0.334 & -1.033 & -0.698 & 0.512 \\
\hline & 1.629 & 3.462 & 1.251 & 1.354 & 0.648 & 0.784 & 1.233 & 1.698 & 1.039 \\
\hline \multirow[t]{2}{*}{$\mathrm{E}$} & 0.359 & -0.140 & 0.164 & -0.568 & 0.808 & -0.157 & -0.190 & -0.550 & -0.059 \\
\hline & 1.408 & 1.255 & 0.892 & 3.090 & 3.382 & 0.876 & 1.711 & 2.069 & 0.280 \\
\hline \multirow[t]{2}{*}{$\mathrm{S}$} & 0.077 & -0.672 & 0.227 & -0.205 & -0.111 & 0.077 & 0.485 & -0.208 & -0.476 \\
\hline & 0.192 & 1.893 & 1.010 & 1.040 & 0.215 & 0.454 & 1.135 & 1.169 & 1.167 \\
\hline \multirow[t]{2}{*}{$\mathrm{Y}$} & 0.805 & 1.429 & 1.482 & 0.650 & 1.242 & 2.233 & 1.525 & 0.334 & 0.807 \\
\hline & 17.540 & 8.055 & 15.554 & 7.016 & 12.258 & 8.075 & 5.221 & 1.982 & 5.765 \\
\hline \multirow[b]{2}{*}{ Equation } & & & & & REN0 & & & & \\
\hline & $\mathrm{F}$ & A & $\mathrm{C}$ & G & $\mathrm{O}$ & $\mathrm{P}$ & $\mathrm{H}$ & $\mathrm{E}$ & $\mathrm{S}$ \\
\hline \multirow[t]{2}{*}{$\mathrm{F}$} & -0.728 & -0.132 & -0.334 & -0.019 & 0.065 & -0.163 & -0.030 & 0.454 & -0.003 \\
\hline & 3.566 & 1.597 & 4.219 & 0.269 & 0.457 & 3.133 & 0.695 & 4.812 & 0.033 \\
\hline \multirow[t]{2}{*}{ A } & -1.189 & -2.797 & 0.177 & 1.130 & 1.769 & -0.861 & -0.106 & 1.306 & -0.496 \\
\hline & 1.761 & 3.967 & 0.592 & 4.096 & 2.497 & 3.231 & 0.371 & 3.346 & 1.365 \\
\hline \multirow[t]{2}{*}{ C } & -1.426 & 0.050 & -1.619 & -0.251 & 1.489 & -0.058 & 0.152 & 0.085 & 0.045 \\
\hline & 5.267 & 0.402 & 7.464 & 1.869 & 6.685 & 0.550 & 2.072 & 0.522 & 0.104 \\
\hline \multirow[t]{2}{*}{$\mathrm{G}$} & -0.055 & 0.464 & -0.166 & -0.385 & -0.981 & 0.111 & 0.096 & -0.296 & 0.354 \\
\hline & 0.237 & 4.139 & 1.282 & 2.699 & 4.199 & 1.199 & 1.470 & 2.205 & 2.533 \\
\hline \multirow[t]{2}{*}{$\mathrm{O}$} & 0.074 & 0.526 & 1.125 & -0.759 & -1.337 & 0.468 & -0.013 & -0.889 & -0.286 \\
\hline & 0.217 & 2.497 & 7.199 & 4.442 & 3.137 & 3.931 & 0.091 & 4.990 & 1.782 \\
\hline \multirow[t]{2}{*}{$\mathrm{P}$} & -1.966 & -1.116 & -0.169 & 0.287 & 1.931 & -1.663 & 0.118 & 1.343 & -0.194 \\
\hline & 2.802 & 3.061 & 0.488 & 0.981 & 2.719 & 4.339 & 0.725 & 2.726 & 0.491 \\
\hline \multirow[t]{2}{*}{$\mathrm{H}$} & -0.735 & -0.316 & 1.208 & 0.763 & -0.074 & 0.309 & 0.569 & -1.789 & -0.686 \\
\hline & 0.597 & 0.352 & 1.944 & 1.503 & 0.045 & 0.751 & 0.472 & 2.382 & 0.798 \\
\hline \multirow[t]{2}{*}{$\mathrm{E}$} & 2.593 & 0.881 & 0.235 & -0.478 & -1.906 & 0.725 & -0.381 & -2.751 & 0.344 \\
\hline & 5.068 & 3.464 & 0.944 & 2.179 & 4.013 & 3.473 & 3.027 & 5.954 & 1.365 \\
\hline \multirow[t]{2}{*}{$\mathrm{S}$} & 0.054 & -0.834 & 0.275 & 1.551 & -1.569 & -0.237 & -0.378 & 0.913 & -0.400 \\
\hline & 0.075 & 1.347 & 0.760 & 4.614 & 1.896 & 0.915 & 0.819 & 2.477 & 0.541 \\
\hline $\mathrm{Y}$ & 0.890 & 1.067 & 1.532 & 0.857 & 1.092 & 1.429 & 0.752 & 0.739 & 0.623 \\
\hline & 30.925 & 8.968 & 21.121 & 18.294 & 17.264 & 11.073 & 4.051 & 8.109 & 4.982 \\
\hline
\end{tabular}


Table 15: FACGOPHES System Elasticity Estimates, MOR1 and REN1 Households. Estimates Based on Four-Region Data, 1980-1992.

\begin{tabular}{|c|c|c|c|c|c|c|c|c|c|}
\hline & & & & & $\overline{\overline{\text { MOR1 }}}$ & & & & \\
\hline Equation & $\mathrm{F}$ & A & $\mathrm{C}$ & G & $\mathrm{O}$ & $\mathrm{P}$ & $\mathrm{H}$ & $\mathrm{E}$ & $\mathrm{S}$ \\
\hline F & -1.145 & -0.069 & 0.144 & -0.030 & -0.055 & 0.059 & -0.152 & 0.369 & 0.107 \\
\hline & 6.536 & 1.124 & 2.065 & 0.522 & 0.447 & 1.061 & 1.471 & 5.529 & 0.574 \\
\hline A & -0.684 & 0.493 & -0.370 & 1.170 & 0.002 & 0.026 & 1.408 & -1.591 & -0.850 \\
\hline & 0.971 & 0.706 & 1.015 & 3.328 & 0.002 & 0.077 & 2.033 & 5.048 & 1.310 \\
\hline C & 0.170 & -0.132 & -1.313 & -0.002 & 0.595 & -0.073 & -0.129 & -0.343 & -0.268 \\
\hline & 0.788 & 1.384 & 8.128 & 0.018 & 3.191 & 0.859 & 0.786 & 3.455 & 0.662 \\
\hline G & -0.034 & 0.341 & 0.105 & -0.769 & -0.705 & 0.275 & 0.505 & -0.281 & -0.027 \\
\hline & 0.170 & 3.284 & 0.977 & 5.841 & 3.352 & 3.188 & 2.763 & 2.872 & 0.079 \\
\hline $\mathrm{O}$ & -0.363 & -0.034 & 0.506 & -0.586 & -1.630 & 0.280 & 0.022 & 0.373 & 0.065 \\
\hline & 1.211 & 0.201 & 3.357 & 3.951 & 4.090 & 2.211 & 0.076 & 2.515 & 0.204 \\
\hline $\mathrm{P}$ & 0.731 & 0.013 & -0.318 & 1.102 & 1.676 & -2.928 & -0.782 & -0.682 & 0.051 \\
\hline & 0.603 & 0.023 & 0.793 & 2.465 & 2.217 & 6.063 & 1.111 & 1.369 & 0.162 \\
\hline $\mathrm{H}$ & -1.109 & 0.645 & -0.237 & 0.738 & 0.030 & -0.311 & -1.182 & -0.543 & 0.517 \\
\hline & 1.369 & 1.633 & 0.768 & 2.206 & 0.046 & 1.216 & 1.374 & 1.411 & 1.113 \\
\hline $\mathrm{E}$ & 1.477 & -0.611 & -0.426 & -0.407 & 0.716 & -0.200 & -0.383 & -1.154 & -0.058 \\
\hline & 3.980 & 3.708 & 3.056 & 2.737 & 2.782 & 1.725 & 1.693 & 3.663 & 0.596 \\
\hline $\mathrm{S}$ & 1.627 & -1.100 & -1.247 & -0.132 & 0.495 & 0.061 & 1.418 & -0.172 & -1.699 \\
\hline & 2.624 & 1.990 & 3.536 & 0.420 & 0.669 & 0.213 & 1.892 & 0.571 & 2.311 \\
\hline Y & 0.773 & 0.396 & 1.494 & 0.591 & 1.367 & 1.137 & 1.452 & 1.046 & 0.750 \\
\hline & 19.251 & 1.791 & 19.289 & 9.015 & 14.288 & 4.706 & 9.036 & 12.738 & 4.223 \\
\hline & & & & & REN1 & & & & \\
\hline Equation & $\mathrm{F}$ & A & $\mathrm{C}$ & G & $\mathrm{O}$ & $\mathrm{P}$ & $\mathrm{H}$ & E & $\mathrm{S}$ \\
\hline $\mathrm{F}$ & -0.966 & 0.101 & 0.159 & -0.073 & 0.092 & 0.175 & -0.189 & 0.023 & 0.035 \\
\hline & 7.324 & 1.781 & 2.832 & 1.472 & 0.890 & 4.380 & 2.248 & 0.389 & 0.317 \\
\hline A & 0.629 & -2.415 & -0.626 & 0.829 & -0.164 & -0.410 & 0.652 & -0.015 & -0.502 \\
\hline & 0.908 & 2.887 & 1.603 & 2.161 & 0.192 & 1.465 & 0.864 & 0.038 & 1.018 \\
\hline C & 0.308 & -0.173 & -1.704 & 0.152 & 0.012 & -0.316 & 0.319 & 0.303 & -0.248 \\
\hline & 1.472 & 1.428 & 9.710 & 1.375 & 0.061 & 4.058 & 1.978 & 2.452 & 2.064 \\
\hline G & -0.217 & 0.292 & 0.225 & -0.830 & 0.077 & -0.216 & 0.689 & -0.517 & -0.078 \\
\hline & 1.209 & 2.656 & 2.180 & 6.953 & 0.372 & 2.854 & 4.974 & 4.723 & 0.549 \\
\hline $\mathrm{O}$ & 0.000 & -0.014 & 0.025 & -0.020 & -1.462 & 0.303 & -0.574 & 0.371 & 0.074 \\
\hline & 0.001 & 0.061 & 0.144 & 0.106 & 2.650 & 2.094 & 1.492 & 1.929 & 0.461 \\
\hline $\mathrm{P}$ & 2.352 & -0.560 & -1.428 & -1.091 & 1.346 & -2.181 & 1.228 & -0.977 & -0.604 \\
\hline & 3.360 & 1.358 & 4.312 & 2.696 & 1.819 & 4.327 & 2.342 & 2.746 & 1.859 \\
\hline $\mathrm{H}$ & -2.021 & 0.430 & 0.625 & 1.412 & -1.483 & 0.625 & -2.868 & 0.661 & 0.775 \\
\hline & 2.802 & 0.849 & 1.833 & 4.171 & 1.596 & 2.376 & 2.764 & 1.719 & 2.071 \\
\hline $\mathrm{E}$ & -0.134 & 0.021 & 0.438 & -0.837 & 0.594 & -0.298 & 0.467 & -1.443 & -0.035 \\
\hline & 0.436 & 0.110 & 2.589 & 4.414 & 1.867 & 2.288 & 1.827 & 5.664 & 0.336 \\
\hline S & 0.453 & -0.733 & -1.191 & -0.466 & 0.432 & -0.673 & 1.827 & -0.116 & -0.652 \\
\hline & 0.915 & 1.256 & 4.310 & 1.615 & 0.589 & 3.502 & 2.907 & 0.407 & 1.022 \\
\hline Y & 0.643 & 2.022 & 1.347 & 0.573 & 1.298 & 1.915 & 1.844 & 1.227 & 1.121 \\
\hline & 13.171 & 8.168 & 14.433 & 6.863 & 9.032 & 5.423 & 7.822 & 8.045 & 6.928 \\
\hline
\end{tabular}


Table 16: FACGOPHES System Elasticity Estimates, MOR2 and REN2 Households. Estimates Based on Four-Region Data, 1980-1992.

\begin{tabular}{|c|c|c|c|c|c|c|c|c|c|}
\hline \multirow[b]{2}{*}{ Equation } & \multicolumn{9}{|c|}{ "MOR2 } \\
\hline & $\mathrm{F}$ & A & C & G & $\mathrm{O}$ & $\mathrm{P}$ & $\mathrm{H}$ & $\mathrm{E}$ & $\mathrm{S}$ \\
\hline \multirow[t]{2}{*}{$\mathrm{F}$} & -0.738 & 0.054 & -0.099 & -0.143 & -0.344 & 0.028 & -0.023 & 0.358 & 0.032 \\
\hline & 4.180 & 1.127 & 1.587 & 2.671 & 2.805 & 0.668 & 0.255 & 5.912 & 0.529 \\
\hline \multirow[t]{2}{*}{ A } & 0.696 & 0.092 & -0.349 & 1.925 & -0.659 & -0.528 & -1.254 & -0.289 & -0.545 \\
\hline & 1.137 & 0.129 & 0.976 & 5.583 & 0.810 & 2.003 & 1.770 & 0.833 & 1.229 \\
\hline \multirow[t]{2}{*}{ C } & -0.532 & -0.102 & -0.765 & -0.286 & 0.449 & 0.070 & -0.032 & -0.303 & 0.060 \\
\hline & 2.822 & 1.202 & 5.208 & 3.016 & 2.689 & 1.164 & 0.243 & 3.390 & 0.317 \\
\hline \multirow[t]{2}{*}{$\mathrm{G}$} & -0.440 & 0.545 & -0.236 & -0.159 & -0.810 & 0.187 & 0.410 & -0.239 & 0.092 \\
\hline & 2.310 & 5.587 & 2.125 & 1.252 & 3.724 & 2.636 & 2.761 & 2.684 & 0.583 \\
\hline \multirow[t]{2}{*}{$\mathrm{O}$} & -1.121 & -0.152 & 0.414 & -0.682 & 0.248 & 0.241 & -0.216 & 0.013 & -0.029 \\
\hline & 3.465 & 0.883 & 2.751 & 4.208 & 0.591 & 2.283 & 0.783 & 0.081 & 0.185 \\
\hline \multirow[t]{2}{*}{$\mathrm{P}$} & 0.765 & -0.694 & 0.558 & 0.958 & 1.707 & -1.544 & -1.518 & -0.367 & -0.053 \\
\hline & 0.987 & 1.869 & 1.519 & 2.791 & 2.474 & 3.367 & 2.494 & 0.772 & 0.124 \\
\hline \multirow[t]{2}{*}{$\mathrm{H}$} & -0.450 & -0.582 & -0.081 & 0.544 & -0.501 & -0.530 & -0.007 & -0.158 & 0.087 \\
\hline & 0.816 & 1.812 & 0.296 & 2.279 & 0.836 & 2.307 & 0.010 & 0.441 & 0.253 \\
\hline \multirow[t]{2}{*}{$\mathrm{E}$} & 1.878 & -0.099 & -0.367 & -0.333 & 0.122 & -0.114 & -0.052 & -1.610 & -0.050 \\
\hline & 6.100 & 0.740 & 2.345 & 2.682 & 0.422 & 0.958 & 0.230 & 5.555 & 0.281 \\
\hline \multirow[t]{2}{*}{$\mathrm{S}$} & 0.468 & -0.608 & 0.359 & 0.341 & -0.089 & -0.060 & 0.282 & -0.169 & -1.393 \\
\hline & 1.089 & 1.233 & 1.491 & 1.443 & 0.166 & 0.299 & 0.572 & 0.682 & 2.784 \\
\hline \multirow[t]{2}{*}{$\mathrm{Y}$} & 0.875 & 0.911 & 1.440 & 0.649 & 1.283 & 0.188 & 1.678 & 0.627 & 0.870 \\
\hline & 20.281 & 4.728 & 16.745 & 8.662 & 11.353 & 0.647 & 9.952 & 6.913 & 4.971 \\
\hline \multirow[b]{2}{*}{ Equation } & & & & & REN2 & & & & \\
\hline & $\mathrm{F}$ & A & C & G & $\mathrm{O}$ & $\mathrm{P}$ & $\mathrm{H}$ & $\mathrm{E}$ & $\mathrm{S}$ \\
\hline \multirow[t]{2}{*}{$\mathrm{F}$} & -1.088 & -0.113 & 0.088 & -0.088 & 0.173 & -0.159 & -0.054 & 0.228 & 0.164 \\
\hline & 6.801 & 2.284 & 1.360 & 1.678 & 1.577 & 3.613 & 0.594 & 4.327 & 2.282 \\
\hline \multirow[t]{2}{*}{ A } & -1.250 & -1.537 & 2.098 & 0.682 & 2.736 & -0.519 & -0.976 & -0.520 & -1.003 \\
\hline & 1.956 & 2.007 & 5.617 & 2.102 & 2.590 & 1.527 & 1.423 & 1.777 & 1.707 \\
\hline \multirow[t]{2}{*}{ C } & 0.226 & 0.597 & -1.655 & 0.158 & -0.522 & -0.086 & 0.305 & 0.038 & -0.164 \\
\hline & 0.889 & 5.271 & 7.237 & 1.340 & 2.385 & 0.907 & 1.569 & 0.338 & 1.970 \\
\hline \multirow[t]{2}{*}{ G } & -0.363 & 0.205 & 0.213 & -0.897 & 0.530 & -0.315 & 0.373 & -0.496 & -0.041 \\
\hline & 1.617 & 1.930 & 1.669 & 6.809 & 2.092 & 3.770 & 2.030 & 4.967 & 0.386 \\
\hline \multirow[t]{2}{*}{$\mathrm{O}$} & 0.259 & 0.762 & -0.609 & 0.370 & -1.675 & 0.806 & -1.754 & 0.149 & -0.102 \\
\hline & 0.606 & 2.423 & 2.791 & 1.592 & 2.122 & 4.251 & 3.824 & 0.813 & 0.267 \\
\hline \multirow[t]{2}{*}{$\mathrm{P}$} & -3.020 & -0.760 & -0.394 & -1.384 & 3.972 & -1.106 & 2.441 & 0.325 & -0.986 \\
\hline & 3.509 & 1.523 & 0.790 & 3.618 & 4.359 & 2.150 & 3.568 & 1.000 & 4.020 \\
\hline \multirow[t]{2}{*}{$\mathrm{H}$} & -0.685 & -0.649 & 0.603 & 0.649 & -3.659 & 1.058 & 1.624 & -0.950 & 0.631 \\
\hline & 0.875 & 1.465 & 1.436 & 1.806 & 3.671 & 3.358 & 1.867 & 3.434 & 1.826 \\
\hline \multirow[t]{2}{*}{$\mathrm{E}$} & 1.454 & -0.296 & 0.071 & -0.808 & 0.352 & 0.113 & -0.760 & -1.227 & 0.048 \\
\hline & 3.723 & 1.770 & 0.341 & 5.159 & 1.120 & 0.938 & 3.365 & 5.194 & 0.489 \\
\hline \multirow[t]{2}{*}{$\mathrm{S}$} & 3.577 & -1.597 & -0.781 & -0.165 & -0.364 & -1.078 & 1.642 & 0.191 & -1.884 \\
\hline & 5.282 & 2.002 & 2.108 & 0.579 & 0.383 & 3.908 & 2.271 & 0.718 & 2.328 \\
\hline $\mathrm{Y}$ & 0.850 & 0.291 & 1.104 & 0.792 & 1.794 & 0.912 & 1.378 & 1.053 & 0.458 \\
\hline & 33.853 & 1.744 & 19.417 & 16.777 & 19.046 & 5.641 & 9.347 & 13.721 & 3.739 \\
\hline
\end{tabular}


Table 17: FACGOPHES System Elasticity Estimates, MOR0 and REN0 Households.

Estimates Based on Three-Region Data, 1980-1992.

\begin{tabular}{|c|c|c|c|c|c|c|c|c|c|}
\hline \multirow[b]{2}{*}{ Equation } & \multicolumn{9}{|c|}{ 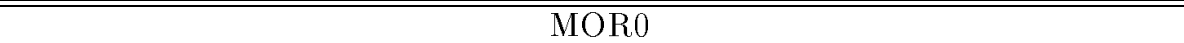 } \\
\hline & $\mathrm{F}$ & A & $\mathrm{C}$ & G & $\mathrm{O}$ & $\mathrm{P}$ & $\mathrm{H}$ & $\mathrm{E}$ & $\mathrm{S}$ \\
\hline \multirow[t]{2}{*}{$\mathrm{F}$} & -1.130 & -0.083 & 0.151 & 0.016 & 0.003 & -0.047 & -0.014 & 0.069 & 0.049 \\
\hline & 6.883 & 1.522 & 1.932 & 0.271 & 0.024 & 0.826 & 0.262 & 1.140 & 1.583 \\
\hline \multirow[t]{2}{*}{ A } & -0.574 & -1.750 & -0.765 & -0.218 & 2.147 & -0.525 & 1.884 & -0.094 & -0.470 \\
\hline & 1.189 & 3.503 & 2.647 & 0.921 & 3.996 & 2.198 & 5.724 & 0.386 & 1.847 \\
\hline \multirow[t]{2}{*}{ C } & 0.344 & -0.288 & -1.286 & 0.077 & -0.027 & 0.132 & -0.153 & -0.117 & 0.027 \\
\hline & 1.557 & 3.169 & 7.804 & 0.734 & 0.155 & 1.480 & 2.070 & 1.122 & 0.497 \\
\hline \multirow[t]{2}{*}{$\mathrm{G}$} & 0.211 & -0.076 & 0.195 & -0.220 & -0.772 & 0.227 & 0.140 & -0.253 & 0.028 \\
\hline & 1.093 & 0.893 & 1.653 & 1.748 & 4.106 & 2.478 & 1.760 & 2.764 & 0.487 \\
\hline \multirow[t]{2}{*}{$\mathrm{O}$} & -0.091 & 0.514 & -0.024 & -0.650 & -1.070 & 0.167 & -0.262 & 0.197 & -0.054 \\
\hline & 0.365 & 3.695 & 0.169 & 4.794 & 2.983 & 1.433 & 1.712 & 1.582 & 0.515 \\
\hline \multirow[t]{2}{*}{$\mathrm{P}$} & -0.610 & -0.659 & 0.496 & 0.639 & 0.765 & -2.561 & -0.170 & 0.343 & 0.490 \\
\hline & 1.052 & 2.384 & 1.469 & 1.877 & 1.445 & 6.002 & 0.679 & 1.220 & 2.913 \\
\hline \multirow[t]{2}{*}{$\mathrm{H}$} & -0.352 & 2.913 & -0.795 & 0.536 & -1.626 & -0.243 & -0.696 & -1.181 & 0.033 \\
\hline & 0.480 & 5.560 & 2.134 & 1.392 & 1.702 & 0.747 & 0.899 & 3.368 & 0.079 \\
\hline \multirow[t]{2}{*}{$\mathrm{E}$} & 0.336 & -0.063 & -0.120 & -0.373 & 0.416 & 0.152 & -0.339 & -0.890 & -0.005 \\
\hline & 1.371 & 0.528 & 0.740 & 2.613 & 1.785 & 1.321 & 3.096 & 4.954 & 0.077 \\
\hline \multirow[t]{2}{*}{$\mathrm{S}$} & 0.705 & -0.701 & 0.193 & 0.062 & -0.222 & 0.627 & 0.047 & -0.014 & -1.541 \\
\hline & 1.774 & 1.910 & 0.772 & 0.274 & 0.380 & 2.970 & 0.120 & 0.074 & 3.863 \\
\hline \multirow[t]{2}{*}{$\mathrm{Y}$} & 0.987 & 0.364 & 1.290 & 0.520 & 1.273 & 1.268 & 1.409 & 0.885 & 0.845 \\
\hline & 15.648 & 1.502 & 8.799 & 4.172 & 10.226 & 3.217 & 3.885 & 4.505 & 5.205 \\
\hline \multirow[b]{2}{*}{ Equation } & & & & & REN0 & & & & \\
\hline & $\mathrm{F}$ & A & $\mathrm{C}$ & $\mathrm{G}$ & $\mathrm{O}$ & $\mathrm{P}$ & $\mathrm{H}$ & $\mathrm{E}$ & $\mathrm{S}$ \\
\hline \multirow[t]{2}{*}{$\mathrm{F}$} & -0.379 & -0.032 & 0.019 & -0.077 & -0.317 & -0.090 & -0.126 & 0.148 & -0.047 \\
\hline & 1.054 & 0.203 & 0.101 & 0.552 & 0.923 & 0.618 & 1.153 & 0.948 & 0.079 \\
\hline \multirow[t]{2}{*}{ A } & -0.214 & -2.767 & -0.151 & 1.950 & -2.222 & 0.022 & 1.497 & 0.765 & 0.366 \\
\hline & 0.158 & 2.036 & 0.169 & 2.835 & 1.144 & 0.029 & 1.875 & 0.913 & 0.227 \\
\hline \multirow[t]{2}{*}{ C } & -0.124 & -0.087 & -1.324 & -0.161 & 0.724 & -0.822 & 0.082 & 0.403 & -0.061 \\
\hline & 0.193 & 0.245 & 2.906 & 0.548 & 1.036 & 2.818 & 0.342 & 1.259 & 0.028 \\
\hline \multirow[t]{2}{*}{$\mathrm{G}$} & -0.303 & 0.783 & -0.120 & 0.325 & -1.874 & 0.267 & 0.347 & -0.385 & -0.018 \\
\hline & 0.615 & 2.798 & 0.400 & 0.900 & 2.765 & 1.016 & 1.721 & 1.320 & 0.083 \\
\hline \multirow[t]{2}{*}{$\mathrm{O}$} & -0.870 & -0.662 & 0.567 & -1.352 & 2.267 & 0.144 & -0.635 & -0.670 & 0.165 \\
\hline & 1.001 & 1.172 & 1.116 & 2.797 & 1.549 & 0.321 & 1.473 & 1.455 & 0.369 \\
\hline \multirow[t]{2}{*}{$\mathrm{P}$} & -1.058 & 0.011 & -2.487 & 0.787 & 0.586 & -2.216 & 1.491 & 1.793 & -0.042 \\
\hline & 0.286 & 0.008 & 0.299 & 0.501 & 0.188 & 0.172 & 0.624 & 0.226 & 0.024 \\
\hline \multirow[t]{2}{*}{$\mathrm{H}$} & -3.431 & 4.658 & 0.693 & 2.669 & -6.768 & 3.811 & 2.193 & -3.243 & -1.493 \\
\hline & 0.568 & 1.603 & 0.053 & 1.049 & 1.150 & 0.373 & 0.284 & 0.269 & 0.564 \\
\hline \multirow[t]{2}{*}{$\mathrm{E}$} & 0.771 & 0.453 & 0.665 & -0.566 & -1.367 & 0.895 & -0.629 & -0.833 & -0.309 \\
\hline & 0.326 & 0.488 & 0.116 & 0.525 & 0.699 & 0.196 & 0.381 & 0.078 & 0.293 \\
\hline \multirow[t]{2}{*}{$\mathrm{S}$} & -0.822 & 0.597 & -0.236 & -0.103 & 0.929 & -0.061 & -0.814 & -0.891 & 0.177 \\
\hline & 0.591 & 0.498 & 0.293 & 0.138 & 0.451 & 0.080 & 0.790 & 1.170 & 0.097 \\
\hline $\mathrm{Y}$ & 0.901 & 0.752 & 1.370 & 0.978 & 1.047 & 1.136 & 0.912 & 0.920 & 1.222 \\
\hline & 26.614 & 5.600 & 13.754 & 19.319 & 15.782 & 7.269 & 3.261 & 10.193 & 9.316 \\
\hline
\end{tabular}


Table 18: FACGOPHES System Elasticity Estimates, MOR1 and REN1 Households.

Estimates Based on Three-Region Data, 1980-1992.

\begin{tabular}{|c|c|c|c|c|c|c|c|c|c|}
\hline & & & & & $\overline{\overline{\text { MOR1 }}}$ & & & & \\
\hline Equation & $\mathrm{F}$ & A & $\mathrm{C}$ & G & $\mathrm{O}$ & $\mathrm{P}$ & $\mathrm{H}$ & $\mathrm{E}$ & $\mathrm{S}$ \\
\hline F & -1.187 & 0.003 & 0.101 & -0.124 & 0.095 & -0.242 & 0.145 & 0.338 & 0.105 \\
\hline & 5.558 & 0.044 & 1.004 & 1.778 & 0.627 & 3.282 & 1.115 & 4.178 & 0.296 \\
\hline A & 0.107 & -1.040 & -0.341 & 0.557 & -0.308 & 0.253 & 1.655 & -0.822 & -0.639 \\
\hline & 0.130 & 1.523 & 0.771 & 1.420 & 0.367 & 0.621 & 2.330 & 2.138 & 0.899 \\
\hline C & 0.097 & -0.109 & -1.133 & 0.538 & 0.284 & -0.070 & -0.418 & -0.260 & -0.231 \\
\hline & 0.318 & 0.967 & 5.344 & 4.132 & 1.139 & 0.649 & 2.032 & 2.114 & 0.501 \\
\hline G & -0.332 & 0.165 & 0.733 & -1.235 & -0.576 & 0.370 & 0.704 & -0.311 & -0.020 \\
\hline & 1.405 & 1.443 & 4.933 & 6.719 & 2.180 & 3.111 & 3.317 & 2.386 & 0.026 \\
\hline $\mathrm{O}$ & -0.037 & -0.096 & 0.218 & -0.538 & -1.609 & 0.465 & -0.411 & 0.455 & 0.055 \\
\hline & 0.096 & 0.531 & 1.037 & 2.719 & 3.066 & 2.411 & 1.104 & 2.539 & 0.072 \\
\hline $\mathrm{P}$ & -3.764 & 0.304 & -0.380 & 1.533 & 2.722 & -4.365 & 1.626 & 0.881 & 0.081 \\
\hline & 1.322 & 0.578 & 0.609 & 2.409 & 1.779 & 1.594 & 0.916 & 0.877 & 0.122 \\
\hline $\mathrm{H}$ & 0.724 & 0.810 & -0.787 & 1.127 & -0.867 & 0.648 & -1.552 & -1.375 & 0.232 \\
\hline & 0.461 & 2.370 & 1.743 & 2.627 & 0.841 & 0.915 & 0.863 & 2.413 & 0.852 \\
\hline $\mathrm{E}$ & 1.226 & -0.326 & -0.381 & -0.482 & 0.795 & 0.258 & -1.031 & -1.409 & 0.015 \\
\hline & 1.807 & 2.213 & 2.026 & 2.760 & 2.062 & 0.850 & 2.439 & 3.917 & 0.030 \\
\hline $\mathrm{S}$ & 1.481 & -0.841 & -1.164 & -0.139 & 0.372 & 0.088 & 0.597 & 0.068 & -1.545 \\
\hline & 2.473 & 2.024 & 2.978 & 0.462 & 0.560 & 0.274 & 0.971 & 0.255 & 2.855 \\
\hline Y & 0.766 & 0.578 & 1.302 & 0.502 & 1.499 & 1.361 & 1.040 & 1.335 & 1.082 \\
\hline & 20.593 & 3.207 & 20.417 & 7.114 & 16.692 & 6.492 & 7.149 & 17.652 & 8.849 \\
\hline & & & & & REN1 & & & & \\
\hline Equation & $\mathrm{F}$ & A & $\mathrm{C}$ & G & $\mathrm{O}$ & $\mathrm{P}$ & $\mathrm{H}$ & E & $\mathrm{S}$ \\
\hline $\mathrm{F}$ & -0.976 & -0.006 & 0.106 & 0.022 & -0.058 & 0.252 & -0.140 & 0.143 & 0.033 \\
\hline & 3.272 & 0.047 & 0.851 & 0.221 & 0.273 & 2.520 & 0.842 & 1.202 & 0.536 \\
\hline A & -0.527 & 3.198 & -1.633 & 1.157 & 0.197 & 0.609 & -1.570 & -2.708 & -0.547 \\
\hline & 0.311 & 1.564 & 1.638 & 1.426 & 0.085 & 0.789 & 0.840 & 2.746 & 0.621 \\
\hline C & 0.154 & -0.485 & -1.639 & -0.451 & 0.476 & -0.263 & 0.881 & 0.384 & -0.283 \\
\hline & 0.305 & 1.548 & 4.502 & 2.091 & 0.983 & 1.218 & 2.321 & 1.435 & 1.938 \\
\hline G & 0.191 & 0.386 & -0.326 & -0.815 & -0.001 & -0.012 & 0.690 & -0.422 & -0.018 \\
\hline & 0.535 & 1.624 & 1.627 & 3.246 & 0.002 & 0.072 & 2.278 & 1.975 & 0.137 \\
\hline $\mathrm{O}$ & -0.512 & 0.085 & 0.429 & -0.132 & -0.155 & 0.025 & -1.783 & 0.802 & -0.013 \\
\hline & 0.682 & 0.133 & 1.000 & 0.312 & 0.111 & 0.070 & 1.987 & 1.908 & 0.035 \\
\hline $\mathrm{P}$ & 3.847 & 0.810 & -1.162 & -0.161 & 0.051 & -1.498 & -0.842 & -2.320 & -0.246 \\
\hline & 1.674 & 0.716 & 1.226 & 0.065 & 0.027 & 0.800 & 0.367 & 1.403 & 0.510 \\
\hline $\mathrm{H}$ & -2.224 & -1.086 & 1.712 & 1.250 & -4.653 & -0.447 & 1.316 & 0.176 & 0.839 \\
\hline & 1.294 & 0.847 & 2.047 & 0.860 & 2.111 & 0.569 & 0.561 & 0.148 & 1.156 \\
\hline $\mathrm{E}$ & 0.449 & -1.132 & 0.509 & -0.730 & 1.201 & -0.736 & 0.199 & -1.292 & 0.180 \\
\hline & 0.507 & 2.517 & 1.348 & 0.743 & 1.648 & 1.870 & 0.215 & 1.179 & 0.909 \\
\hline S & 0.358 & -0.798 & -1.362 & -0.200 & -0.075 & -0.268 & 1.950 & 0.645 & -1.490 \\
\hline & 0.320 & 0.605 & 1.965 & 0.317 & 0.038 & 0.495 & 1.231 & 0.930 & 1.103 \\
\hline Y & 0.623 & 1.825 & 1.226 & 0.328 & 1.254 & 1.519 & 3.116 & 1.352 & 1.239 \\
\hline & 6.099 & 3.220 & 6.264 & 1.531 & 4.423 & 1.959 & 6.117 & 4.149 & 3.481 \\
\hline
\end{tabular}


Table 19: FACGOPHES System Elasticity Estimates, MOR2 and REN2 Households.

Estimates Based on Three-Region Data, 1980-1992.

\begin{tabular}{|c|c|c|c|c|c|c|c|c|c|}
\hline & & & & & $\overline{\overline{\text { MOR2 }}}$ & & & & \\
\hline Equation & $\mathrm{F}$ & A & $\mathrm{C}$ & G & $\mathrm{O}$ & $\mathrm{P}$ & $\mathrm{H}$ & $\mathrm{E}$ & $\mathrm{S}$ \\
\hline F & -0.866 & -0.031 & 0.169 & 0.110 & -0.159 & -0.130 & 0.075 & 0.108 & -0.038 \\
\hline & 4.104 & 0.443 & 2.157 & 1.878 & 1.167 & 2.651 & 0.745 & 1.425 & 0.261 \\
\hline A & -0.335 & 1.058 & -0.905 & 1.426 & -3.055 & 0.898 & 0.864 & -0.857 & 0.426 \\
\hline & 0.354 & 1.377 & 2.289 & 3.997 & 3.343 & 2.634 & 1.095 & 1.406 & 0.883 \\
\hline C & 0.202 & -0.248 & -0.962 & -0.073 & 0.213 & -0.212 & -0.384 & -0.038 & -0.087 \\
\hline & 0.841 & 2.710 & 4.950 & 0.657 & 1.079 & 2.436 & 2.228 & 0.292 & 0.241 \\
\hline G & 0.455 & 0.395 & 0.026 & 0.071 & -0.700 & 0.007 & -0.377 & -0.558 & 0.013 \\
\hline & 2.138 & 3.891 & 0.199 & 0.485 & 2.925 & 0.084 & 2.254 & 4.751 & 0.062 \\
\hline $\mathrm{O}$ & -0.756 & -0.688 & 0.210 & -0.624 & -0.077 & 0.361 & -0.237 & 0.457 & -0.142 \\
\hline & 1.999 & 3.541 & 1.142 & 3.452 & 0.135 & 2.553 & 0.674 & 2.488 & 0.444 \\
\hline $\mathrm{P}$ & -2.266 & 1.348 & -1.137 & 0.106 & 2.705 & -0.189 & -0.758 & -0.424 & 0.655 \\
\hline & 0.759 & 0.649 & 1.654 & 0.208 & 2.492 & 0.111 & 0.576 & 0.206 & 0.778 \\
\hline $\mathrm{H}$ & 0.149 & 0.372 & -0.784 & -0.737 & -0.541 & -0.276 & -0.163 & -0.080 & 0.468 \\
\hline & 0.066 & 0.228 & 1.625 & 2.126 & 0.637 & 0.389 & 0.081 & 0.051 & 0.841 \\
\hline $\mathrm{E}$ & 0.422 & -0.299 & 0.023 & -0.698 & 0.786 & -0.115 & -0.019 & -1.002 & -0.042 \\
\hline & 0.255 & 0.245 & 0.076 & 3.410 & 2.032 & 0.218 & 0.028 & 0.445 & 0.156 \\
\hline$S$ & -0.540 & 0.467 & -0.288 & 0.046 & -0.609 & 0.475 & 1.152 & -0.116 & -1.246 \\
\hline & 1.133 & 1.156 & 1.099 & 0.216 & 1.050 & 2.072 & 2.160 & 0.391 & 2.656 \\
\hline Y & 0.763 & 0.480 & 1.590 & 0.668 & 1.496 & -0.039 & 1.593 & 0.942 & 0.660 \\
\hline & 15.107 & 1.938 & 16.608 & 7.034 & 11.410 & 0.114 & 6.557 & 6.804 & 4.074 \\
\hline & & & & & REN2 & & & & \\
\hline Equation & $\mathrm{F}$ & A & $\mathrm{C}$ & $\mathrm{G}$ & $\mathrm{O}$ & $\mathrm{P}$ & $\mathrm{H}$ & E & $\mathrm{S}$ \\
\hline $\mathrm{F}$ & -0.589 & -0.134 & 0.003 & 0.028 & -0.011 & -0.193 & -0.019 & 0.147 & 0.123 \\
\hline & 1.801 & 0.903 & 0.020 & 0.275 & 0.053 & 1.732 & 0.112 & 1.151 & 0.771 \\
\hline A & -1.914 & -0.287 & -0.015 & 2.173 & 1.069 & 1.010 & 0.102 & 0.174 & -2.911 \\
\hline & 0.929 & 0.109 & 0.012 & 2.249 & 0.421 & 1.060 & 0.047 & 0.149 & 2.711 \\
\hline C & -0.327 & -0.033 & -0.947 & 0.003 & -0.086 & 0.124 & -0.327 & 0.273 & -0.040 \\
\hline & 0.543 & 0.101 & 1.911 & 0.011 & 0.194 & 0.482 & 0.863 & 0.922 & 0.197 \\
\hline G & 0.132 & 0.725 & 0.089 & -0.860 & 0.036 & -0.012 & 0.080 & -1.067 & 0.222 \\
\hline & 0.284 & 2.223 & 0.303 & 2.795 & 0.075 & 0.055 & 0.211 & 3.787 & 1.053 \\
\hline $\mathrm{O}$ & -0.361 & 0.283 & -0.092 & -0.034 & -1.019 & 0.306 & -0.180 & 0.343 & -0.577 \\
\hline & 0.446 & 0.384 & 0.190 & 0.081 & 0.704 & 0.765 & 0.191 & 0.812 & 1.338 \\
\hline $\mathrm{P}$ & -3.916 & 1.148 & 0.460 & -0.178 & 1.175 & 0.309 & 0.575 & -0.858 & -0.781 \\
\hline & 2.143 & 0.671 & 0.377 & 0.202 & 0.665 & 0.252 & 0.412 & 0.628 & 0.891 \\
\hline $\mathrm{H}$ & -0.911 & 0.005 & -0.972 & 0.016 & -0.548 & 0.313 & -0.352 & -0.596 & 0.724 \\
\hline & 0.564 & 0.003 & 0.941 & 0.019 & 0.239 & 0.414 & 0.152 & 0.496 & 0.717 \\
\hline $\mathrm{E}$ & 0.468 & 0.038 & 0.410 & -1.507 & 0.487 & -0.306 & -0.345 & -1.321 & 0.446 \\
\hline & 0.595 & 0.046 & 0.761 & 3.412 & 0.670 & 0.837 & 0.574 & 1.224 & 1.149 \\
\hline $\mathrm{S}$ & 2.560 & -4.313 & -0.112 & 0.984 & -2.853 & -0.920 & 1.664 & 1.585 & 0.818 \\
\hline & 1.868 & 2.740 & 0.144 & 1.418 & 1.363 & 1.434 & 1.012 & 2.246 & 0.572 \\
\hline Y & 0.645 & 0.600 & 1.359 & 0.656 & 1.331 & 2.066 & 2.320 & 1.631 & 0.587 \\
\hline & 7.725 & 1.052 & 5.896 & 3.264 & 5.851 & 3.138 & 4.764 & 5.047 & 1.637 \\
\hline
\end{tabular}


Table 20: FACGOP System Elasticity Estimates,

MOR0, MOR1 and MOR2 Households.

Estimates Based on Four-Region Data, 1980-1985.

\begin{tabular}{|c|c|c|c|c|c|c|}
\hline \multirow[b]{2}{*}{ Equation } & \multicolumn{6}{|c|}{ MOR0 } \\
\hline & $\mathrm{F}$ & A & $\mathrm{C}$ & $\mathrm{G}$ & $\mathrm{O}$ & $\mathrm{P}$ \\
\hline \multirow[t]{2}{*}{$\mathrm{F}$} & -0.543 & -0.054 & 0.038 & 0.082 & -0.150 & -0.157 \\
\hline & 2.755 & 0.542 & 0.548 & 0.954 & 1.308 & 2.839 \\
\hline \multirow[t]{2}{*}{ A } & -0.498 & -0.525 & -0.338 & -0.829 & 0.556 & 0.490 \\
\hline & 0.795 & 0.886 & 1.100 & 2.301 & 1.081 & 2.726 \\
\hline \multirow[t]{2}{*}{ C } & -0.297 & -0.201 & -1.268 & -0.113 & 0.050 & 0.090 \\
\hline & 1.425 & 1.359 & 6.589 & 0.657 & 0.299 & 0.730 \\
\hline \multirow[t]{2}{*}{ G } & 0.444 & -0.299 & 0.119 & -0.595 & 0.083 & -0.014 \\
\hline & 1.888 & 1.929 & 0.779 & 2.546 & 0.436 & 0.096 \\
\hline \multirow[t]{2}{*}{$\mathrm{O}$} & -0.504 & 0.236 & 0.136 & -0.037 & -1.202 & 0.335 \\
\hline & 1.674 & 1.109 & 0.969 & 0.205 & 3.638 & 3.458 \\
\hline \multirow[t]{2}{*}{$\mathrm{P}$} & -2.108 & 0.528 & 0.054 & -0.404 & 0.785 & -1.580 \\
\hline & 4.687 & 2.292 & 0.162 & 0.957 & 2.598 & 3.980 \\
\hline \multirow[t]{2}{*}{ Y } & 0.784 & 1.144 & 1.740 & 0.263 & 1.036 & 2.725 \\
\hline & 16.566 & 5.643 & 14.492 & 2.968 & 10.262 & 9.447 \\
\hline & \multicolumn{6}{|c|}{ MOR1 } \\
\hline Equation & $\mathrm{F}$ & A & C & $\mathrm{G}$ & $\mathrm{O}$ & $\mathrm{P}$ \\
\hline \multirow[t]{2}{*}{$\mathrm{F}$} & -0.101 & -0.417 & -0.159 & -0.042 & -0.008 & -0.187 \\
\hline & 0.632 & 5.123 & 2.271 & 0.609 & 0.060 & 4.082 \\
\hline \multirow[t]{2}{*}{ A } & -3.072 & 1.899 & 0.151 & 0.283 & 0.026 & 0.127 \\
\hline & 4.939 & 3.399 & 0.714 & 0.798 & 0.040 & 0.670 \\
\hline \multirow[t]{2}{*}{$\mathrm{C}$} & -0.851 & 0.000 & -1.216 & -0.023 & 0.216 & 0.141 \\
\hline & 4.137 & 0.002 & 5.791 & 0.175 & 1.215 & 1.744 \\
\hline \multirow[t]{2}{*}{ G } & -0.030 & 0.103 & 0.137 & -0.578 & -0.349 & 0.036 \\
\hline & 0.134 & 0.723 & 1.010 & 2.657 & 1.654 & 0.423 \\
\hline \multirow[t]{2}{*}{$\mathrm{O}$} & -0.109 & -0.023 & 0.301 & -0.383 & -1.178 & 0.295 \\
\hline & 0.287 & 0.096 & 1.859 & 2.009 & 3.128 & 2.516 \\
\hline \multirow[t]{2}{*}{$\mathrm{P}$} & -1.904 & 0.179 & 0.612 & 0.148 & 1.216 & -0.920 \\
\hline & 4.012 & 0.679 & 2.217 & 0.526 & 2.763 & 3.356 \\
\hline \multirow[t]{2}{*}{ Y } & 0.915 & 0.586 & 1.734 & 0.682 & 1.097 & 0.668 \\
\hline & 12.413 & 2.230 & 10.469 & 4.687 & 7.382 & 1.839 \\
\hline & \multicolumn{6}{|c|}{ MOR2 } \\
\hline Equation & F & A & C & G & $\mathrm{O}$ & $\mathrm{P}$ \\
\hline \multirow[t]{2}{*}{ F } & -1.118 & 0.114 & 0.146 & 0.024 & 0.007 & -0.050 \\
\hline & 5.195 & 1.293 & 1.580 & 0.266 & 0.053 & 1.024 \\
\hline \multirow[t]{2}{*}{ A } & 0.923 & -0.488 & -0.066 & -0.645 & -0.215 & -0.393 \\
\hline & 1.241 & 0.807 & 0.201 & 1.629 & 0.354 & 1.884 \\
\hline \multirow[t]{2}{*}{ C } & 0.078 & -0.070 & -1.842 & 0.135 & -0.280 & 0.194 \\
\hline & 0.245 & 0.483 & 7.101 & 0.753 & 1.314 & 1.907 \\
\hline \multirow[t]{2}{*}{ G } & 0.275 & -0.234 & 0.328 & -0.425 & -0.110 & -0.254 \\
\hline & 0.926 & 1.476 & 1.983 & 1.989 & 0.463 & 2.999 \\
\hline $\mathrm{O}$ & -0.097 & -0.097 & -0.145 & -0.233 & -0.969 & 0.428 \\
\hline & 0.209 & 0.407 & 0.750 & 0.989 & 2.027 & 3.411 \\
\hline P & -1.038 & -0.702 & 0.718 & -1.257 & 1.730 & -1.181 \\
\hline & 1.473 & 1.976 & 1.879 & 3.484 & 3.232 & 3.941 \\
\hline Y & 0.878 & 0.883 & 1.784 & 0.420 & 1.114 & 1.730 \\
\hline & 12.550 & 2.392 & 10.400 & 2.881 & 6.666 & 3.806 \\
\hline
\end{tabular}


Table 21: FACGOP System Elasticity Estimates

MOR0, MOR1 and MOR2 Households.

Estimates Based on Four-Region Data, 1980-1992.

\begin{tabular}{|c|c|c|c|c|c|c|}
\hline \multirow[b]{2}{*}{ Equation } & \multicolumn{6}{|c|}{ MOR0 } \\
\hline & $\mathrm{F}$ & $\mathrm{A}$ & $\mathrm{C}$ & $\mathrm{G}$ & $\mathrm{O}$ & $\mathrm{P}$ \\
\hline \multirow[t]{2}{*}{$\mathrm{F}$} & -0.508 & -0.060 & 0.021 & -0.005 & 0.022 & -0.132 \\
\hline & 3.774 & 0.910 & 0.341 & 0.088 & 0.220 & 1.227 \\
\hline \multirow[t]{2}{*}{ A } & -0.867 & -1.939 & -0.310 & 0.379 & 1.187 & 0.200 \\
\hline & 1.552 & 3.855 & 1.179 & 1.467 & 2.285 & 0.847 \\
\hline \multirow[t]{2}{*}{ C } & -0.369 & -0.115 & -1.203 & -0.125 & 0.152 & 0.034 \\
\hline & 2.210 & 1.343 & 8.312 & 1.261 & 1.086 & 0.175 \\
\hline \multirow[t]{2}{*}{ G } & 0.140 & 0.189 & 0.068 & -0.162 & -0.596 & 0.076 \\
\hline & 0.783 & 1.969 & 0.604 & 1.237 & 3.460 & 0.346 \\
\hline \multirow[t]{2}{*}{$\mathrm{O}$} & -0.244 & 0.307 & 0.169 & -0.570 & -1.300 & 0.334 \\
\hline & 1.128 & 2.302 & 1.517 & 4.808 & 4.461 & 2.607 \\
\hline \multirow[t]{2}{*}{$\mathrm{P}$} & -2.269 & 0.135 & -0.137 & -0.072 & 1.090 & -1.653 \\
\hline & 5.116 & 0.569 & 0.477 & 0.264 & 2.733 & 2.643 \\
\hline \multirow[t]{2}{*}{ Y } & 0.661 & 1.350 & 1.627 & 0.285 & 1.305 & 2.905 \\
\hline & 11.210 & 5.266 & 11.389 & 2.406 & 10.357 & 7.327 \\
\hline & \multicolumn{6}{|c|}{ MOR1 } \\
\hline Equation & $\mathrm{F}$ & A & C & $\mathrm{G}$ & $\mathrm{O}$ & $\mathrm{P}$ \\
\hline \multirow[t]{2}{*}{$\mathrm{F}$} & -0.648 & -0.099 & -0.067 & -0.001 & 0.174 & -0.127 \\
\hline & 4.404 & 1.236 & 1.024 & 0.023 & 1.243 & 0.846 \\
\hline \multirow[t]{2}{*}{ A } & -0.572 & -2.114 & -0.617 & 0.234 & 3.377 & 0.165 \\
\hline & 0.662 & 2.465 & 1.451 & 0.469 & 3.076 & 0.168 \\
\hline \multirow[t]{2}{*}{ C } & -0.459 & -0.245 & -1.011 & 0.050 & 0.166 & 0.181 \\
\hline & 2.363 & 2.180 & 6.247 & 0.492 & 0.804 & 0.871 \\
\hline \multirow[t]{2}{*}{ G } & 0.064 & 0.033 & 0.167 & -0.339 & -0.578 & 0.033 \\
\hline & 0.290 & 0.211 & 1.433 & 1.944 & 1.929 & 0.133 \\
\hline \multirow[t]{2}{*}{$\mathrm{O}$} & -0.057 & 0.632 & 0.048 & -0.561 & -1.961 & 0.073 \\
\hline & 0.164 & 2.568 & 0.278 & 2.585 & 3.959 & 0.138 \\
\hline \multirow[t]{2}{*}{$\mathrm{P}$} & -2.038 & 0.157 & 0.823 & 0.059 & 0.434 & -0.821 \\
\hline & 3.096 & 0.321 & 2.334 & 0.168 & 0.572 & 1.844 \\
\hline \multirow[t]{3}{*}{ Y } & 0.768 & -0.473 & 1.317 & 0.621 & 1.827 & 1.387 \\
\hline & 13.314 & 1.259 & 12.333 & 6.103 & 13.002 & 4.082 \\
\hline & \multicolumn{6}{|c|}{ MOR2 } \\
\hline Equation & $\mathrm{F}$ & A & $\mathrm{C}$ & G & $\mathrm{O}$ & $\mathrm{P}$ \\
\hline \multirow[t]{2}{*}{$\mathrm{F}$} & -1.045 & 0.063 & 0.142 & -0.260 & 0.051 & 0.002 \\
\hline & 5.696 & 0.788 & 1.388 & 3.669 & 0.452 & 0.029 \\
\hline \multirow[t]{2}{*}{ A } & 0.722 & -3.357 & 0.792 & 2.056 & -0.248 & -1.150 \\
\hline & 0.714 & 3.833 & 1.251 & 3.737 & 0.288 & 2.759 \\
\hline \multirow[t]{2}{*}{$\mathrm{C}$} & 0.459 & 0.204 & -1.973 & 0.210 & -0.068 & 0.148 \\
\hline & 1.439 & 1.301 & 6.691 & 1.323 & 0.294 & 1.330 \\
\hline \multirow[t]{2}{*}{ G } & -0.729 & 0.627 & 0.325 & -0.028 & -0.665 & -0.090 \\
\hline & 2.717 & 3.786 & 1.673 & 0.104 & 2.489 & 0.186 \\
\hline $\mathrm{O}$ & 0.170 & -0.047 & -0.056 & -0.555 & -0.746 & 0.244 \\
\hline & 0.538 & 0.251 & 0.271 & 2.862 & 2.136 & 2.228 \\
\hline P & -0.408 & -1.618 & 0.666 & -0.581 & 1.360 & -1.375 \\
\hline & 0.431 & 3.182 & 1.096 & 1.147 & 1.989 & 1.233 \\
\hline Y & 1.047 & 1.186 & 1.020 & 0.559 & 0.990 & 1.956 \\
\hline & 41.297 & 6.670 & 18.904 & 6.402 & 14.563 & 7.651 \\
\hline
\end{tabular}


Table 22: FACGOP System Elasticity Estimates

MOR0, MOR1 and MOR2 Households.

Estimates Based on Three-Region Data, 1980-1992.

\begin{tabular}{|c|c|c|c|c|c|c|}
\hline \multirow[b]{2}{*}{ Equation } & \multicolumn{6}{|c|}{ MOR0 } \\
\hline & $\mathrm{F}$ & $\mathrm{A}$ & $\mathrm{C}$ & $\mathrm{G}$ & $\mathrm{O}$ & $\mathrm{P}$ \\
\hline \multirow[t]{2}{*}{$\mathrm{F}$} & -0.900 & -0.060 & 0.151 & 0.003 & 0.133 & -0.126 \\
\hline & 5.711 & 0.825 & 2.080 & 0.049 & 1.178 & 1.859 \\
\hline \multirow[t]{2}{*}{ A } & -0.739 & -1.889 & -0.513 & -0.072 & 1.994 & 0.012 \\
\hline & 1.235 & 3.927 & 1.772 & 0.280 & 3.595 & 0.045 \\
\hline \multirow[t]{2}{*}{$\mathrm{C}$} & 0.296 & -0.160 & -1.156 & 0.013 & -0.207 & 0.107 \\
\hline & 1.600 & 1.738 & 7.474 & 0.121 & 1.261 & 1.210 \\
\hline \multirow[t]{2}{*}{ G } & 0.287 & 0.030 & 0.189 & 0.244 & -0.852 & -0.022 \\
\hline & 1.641 & 0.305 & 1.462 & 1.469 & 3.983 & 0.109 \\
\hline \multirow[t]{2}{*}{$\mathrm{O}$} & 0.041 & 0.514 & -0.239 & -0.794 & -1.351 & 0.359 \\
\hline & 0.159 & 3.477 & 1.725 & 5.165 & 3.991 & 2.299 \\
\hline \multirow[t]{2}{*}{$\mathrm{P}$} & -2.417 & -0.098 & -0.011 & -0.479 & 1.271 & -1.564 \\
\hline & 4.663 & 0.311 & 0.036 & 1.477 & 2.313 & 2.588 \\
\hline \multirow[t]{2}{*}{$\mathrm{Y}$} & 0.799 & 1.208 & 1.107 & 0.124 & 1.469 & 3.298 \\
\hline & 13.622 & 4.310 & 6.905 & 0.951 & 10.742 & 7.668 \\
\hline & \multicolumn{6}{|c|}{ MOR1 } \\
\hline Equation & $\mathrm{F}$ & A & C & $\mathrm{G}$ & $\mathrm{O}$ & $\mathrm{P}$ \\
\hline \multirow[t]{2}{*}{$\mathrm{F}$} & -0.259 & -0.269 & -0.399 & -0.040 & 0.218 & -0.125 \\
\hline & 1.584 & 3.372 & 5.030 & 0.668 & 1.759 & 1.221 \\
\hline \multirow[t]{2}{*}{ A } & -2.656 & -0.504 & 0.324 & -0.485 & 3.020 & 0.202 \\
\hline & 3.133 & 0.591 & 0.784 & 1.070 & 2.855 & 0.292 \\
\hline \multirow[t]{2}{*}{ C } & -1.420 & 0.024 & -0.301 & 0.088 & 0.091 & 0.094 \\
\hline & 6.216 & 0.227 & 1.518 & 0.705 & 0.465 & 0.317 \\
\hline \multirow[t]{2}{*}{ G } & 0.109 & -0.155 & 0.284 & -0.265 & -0.407 & 0.099 \\
\hline & 0.528 & 1.115 & 1.960 & 1.155 & 1.332 & 0.215 \\
\hline \multirow[t]{2}{*}{$\mathrm{O}$} & 0.241 & 0.617 & 0.051 & -0.454 & -2.058 & 0.060 \\
\hline & 0.741 & 2.499 & 0.295 & 1.930 & 4.151 & 0.152 \\
\hline \multirow[t]{2}{*}{$\mathrm{P}$} & -2.113 & 0.223 & 0.415 & 0.311 & 0.302 & -0.674 \\
\hline & 2.572 & 0.527 & 1.053 & 0.819 & 0.385 & 1.212 \\
\hline \multirow[t]{3}{*}{ Y } & 0.875 & 0.099 & 1.423 & 0.335 & 1.544 & 1.536 \\
\hline & 12.935 & 0.236 & 11.305 & 2.557 & 9.786 & 3.544 \\
\hline & \multicolumn{6}{|c|}{ MOR2 } \\
\hline Equation & $\mathrm{F}$ & A & $\mathrm{C}$ & G & $\mathrm{O}$ & $\mathrm{P}$ \\
\hline \multirow[t]{2}{*}{$\mathrm{F}$} & -0.715 & -0.025 & 0.050 & -0.035 & 0.049 & -0.072 \\
\hline & 4.308 & 0.468 & 0.469 & 0.679 & 0.462 & 1.608 \\
\hline \multirow[t]{2}{*}{ A } & -0.819 & -1.162 & -0.528 & 0.765 & 0.117 & -0.206 \\
\hline & 1.200 & 2.233 & 1.213 & 2.526 & 0.166 & 0.792 \\
\hline \multirow[t]{2}{*}{ C } & -0.282 & -0.112 & -1.039 & -0.097 & -0.058 & -0.031 \\
\hline & 0.852 & 1.043 & 3.964 & 0.879 & 0.331 & 0.340 \\
\hline \multirow[t]{2}{*}{ G } & -0.117 & 0.264 & 0.017 & -0.031 & -1.039 & 0.171 \\
\hline & 0.575 & 2.867 & 0.120 & 0.225 & 5.729 & 2.171 \\
\hline $\mathrm{O}$ & -0.136 & 0.048 & -0.011 & -0.857 & -0.562 & 0.206 \\
\hline & 0.416 & 0.309 & 0.065 & 6.235 & 1.463 & 1.640 \\
\hline P & -0.998 & -0.252 & 0.050 & 0.947 & 1.570 & -1.356 \\
\hline & 1.119 & 0.664 & 0.094 & 2.563 & 1.990 & 3.432 \\
\hline Y & 0.747 & 1.833 & 1.618 & 0.736 & 1.312 & 0.038 \\
\hline & 10.228 & 5.810 & 9.752 & 4.541 & 7.128 & 0.066 \\
\hline
\end{tabular}


Table 23: FACGOP System Elasticity Estimates

REN0, REN1 and REN2 Households.

Estimates Based on Four-Region Data, 1980-1985.

\begin{tabular}{|c|c|c|c|c|c|c|}
\hline \multirow[b]{2}{*}{ Equation } & \multicolumn{6}{|c|}{ 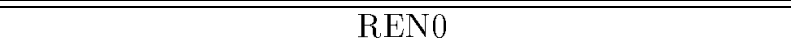 } \\
\hline & $\mathrm{F}$ & $\mathrm{A}$ & $\mathrm{C}$ & $\mathrm{G}$ & $\mathrm{O}$ & $\mathrm{P}$ \\
\hline \multirow[t]{2}{*}{$\mathrm{F}$} & -1.092 & -0.339 & 0.236 & 0.276 & 0.043 & 0.016 \\
\hline & 4.572 & 2.630 & 2.233 & 2.791 & 0.281 & 0.309 \\
\hline \multirow[t]{2}{*}{ A } & -1.959 & 0.506 & 0.135 & 0.666 & -0.578 & 0.612 \\
\hline & 2.518 & 0.563 & 0.382 & 1.664 & 0.926 & 2.885 \\
\hline \multirow[t]{2}{*}{$\mathrm{C}$} & 0.585 & 0.015 & -1.544 & -0.602 & 0.064 & 0.143 \\
\hline & 1.637 & 0.075 & 5.247 & 2.997 & 0.228 & 1.213 \\
\hline \multirow[t]{2}{*}{ G } & 0.771 & 0.306 & -0.499 & -0.189 & -1.360 & -0.041 \\
\hline & 2.409 & 1.486 & 2.796 & 0.766 & 5.407 & 0.348 \\
\hline \multirow[t]{2}{*}{$\mathrm{O}$} & -0.004 & -0.306 & 0.081 & -1.257 & 0.594 & -0.240 \\
\hline & 0.009 & 1.072 & 0.364 & 5.463 & 1.251 & 1.815 \\
\hline \multirow[t]{2}{*}{$\mathrm{P}$} & -0.089 & 0.906 & 0.364 & -0.195 & -0.894 & -1.565 \\
\hline & 0.179 & 2.666 & 1.096 & 0.520 & 1.929 & 5.488 \\
\hline \multirow[t]{2}{*}{ Y } & 0.862 & 0.617 & 1.338 & 1.012 & 1.132 & 1.473 \\
\hline & 18.296 & 4.398 & 11.609 & 15.287 & 14.752 & 10.692 \\
\hline & \multicolumn{6}{|c|}{ REN1 } \\
\hline Equation & $\mathrm{F}$ & $\mathrm{A}$ & $\mathrm{C}$ & $\mathrm{G}$ & $\mathrm{O}$ & $\mathrm{P}$ \\
\hline \multirow[t]{2}{*}{$\mathrm{F}$} & -1.274 & -0.137 & 0.303 & 0.171 & 0.051 & 0.222 \\
\hline & 2.767 & 0.530 & 1.457 & 1.013 & 0.204 & 1.415 \\
\hline \multirow[t]{2}{*}{ A } & -1.619 & 0.785 & -0.688 & -0.089 & -0.114 & -0.093 \\
\hline & 0.817 & 0.415 & 0.813 & 0.094 & 0.080 & 0.149 \\
\hline \multirow[t]{2}{*}{ C } & 0.725 & -0.337 & -1.388 & -0.476 & 0.015 & -0.158 \\
\hline & 0.916 & 0.796 & 2.530 & 1.336 & 0.032 & 0.509 \\
\hline \multirow[t]{2}{*}{ G } & 0.490 & 0.037 & -0.255 & -0.305 & -0.118 & -0.549 \\
\hline & 0.987 & 0.100 & 0.924 & 0.786 & 0.294 & 2.580 \\
\hline \multirow[t]{2}{*}{$\mathrm{O}$} & -0.283 & -0.036 & 0.027 & -0.289 & -1.451 & 0.431 \\
\hline & 0.327 & 0.056 & 0.064 & 0.614 & 1.619 & 1.266 \\
\hline \multirow[t]{2}{*}{$\mathrm{P}$} & 2.678 & -0.109 & -0.450 & -2.356 & 1.616 & -2.366 \\
\hline & 1.979 & 0.119 & 0.606 & 2.980 & 1.646 & 3.304 \\
\hline \multirow[t]{2}{*}{ Y } & 0.664 & 1.817 & 1.619 & 0.700 & 1.602 & 0.988 \\
\hline & 7.429 & 3.620 & 7.482 & 3.745 & 7.095 & 1.724 \\
\hline & \multicolumn{6}{|c|}{$\begin{array}{l}\text { REN2 } \\
\end{array}$} \\
\hline Equation & $\mathrm{F}$ & A & $\mathrm{C}$ & $\mathrm{G}$ & $\mathrm{O}$ & $\mathrm{P}$ \\
\hline \multirow[t]{2}{*}{$\mathrm{F}$} & 0.352 & -0.442 & -0.505 & -0.147 & -0.026 & -0.135 \\
\hline & 0.695 & 1.465 & 2.676 & 0.786 & 0.094 & 0.943 \\
\hline \multirow[t]{2}{*}{ A } & -3.451 & 1.236 & 0.785 & 1.161 & -0.851 & 0.263 \\
\hline & 1.527 & 0.591 & 0.774 & 1.076 & 0.552 & 0.360 \\
\hline \multirow[t]{2}{*}{$\mathrm{C}$} & -2.210 & 0.409 & -0.388 & 0.380 & 0.463 & 0.305 \\
\hline & 2.958 & 0.748 & 0.646 & 0.905 & 0.899 & 1.008 \\
\hline \multirow[t]{2}{*}{$\mathrm{G}$} & -0.698 & 0.532 & 0.315 & -0.542 & -1.024 & 0.220 \\
\hline & 1.023 & 1.011 & 0.832 & 0.984 & 1.945 & 0.714 \\
\hline $\mathrm{O}$ & -0.280 & -0.461 & 0.412 & -1.106 & 0.443 & -0.256 \\
\hline & 0.259 & 0.581 & 0.831 & 1.982 & 0.411 & 0.630 \\
\hline $\mathrm{P}$ & -1.871 & 0.466 & 1.031 & 0.865 & -0.838 & -0.516 \\
\hline & 1.072 & 0.363 & 1.036 & 0.827 & 0.640 & 0.490 \\
\hline Y & 0.904 & 0.856 & 1.042 & 1.197 & 1.248 & 0.864 \\
\hline & 8.302 & 1.249 & 2.978 & 4.216 & 3.958 & 1.111 \\
\hline
\end{tabular}


Table 24: FACGOP System Elasticity Estimates

REN0, REN1 and REN2 Households.

Estimates Based on Four-Region Data, 1980-1992.

\begin{tabular}{|c|c|c|c|c|c|c|}
\hline \multirow[b]{2}{*}{ Equation } & \multicolumn{6}{|c|}{$\overline{\text { REN0 }}$} \\
\hline & $\mathrm{F}$ & A & $\mathrm{C}$ & G & $\mathrm{O}$ & $\mathrm{P}$ \\
\hline \multirow[t]{2}{*}{$\mathrm{F}$} & -0.413 & 0.010 & -0.104 & 0.000 & -0.044 & -0.134 \\
\hline & 2.538 & 0.133 & 1.421 & 0.002 & 0.361 & 2.189 \\
\hline \multirow[t]{2}{*}{ A } & -0.183 & -1.847 & -0.147 & 1.282 & -0.144 & -0.273 \\
\hline & 0.294 & 3.116 & 0.538 & 4.517 & 0.226 & 1.318 \\
\hline \multirow[t]{2}{*}{$\mathrm{C}$} & -0.879 & -0.085 & -1.180 & -0.410 & 0.604 & 0.137 \\
\hline & 3.627 & 0.732 & 5.729 & 2.946 & 2.888 & 1.109 \\
\hline \multirow[t]{2}{*}{ G } & -0.043 & 0.560 & -0.254 & -0.026 & -1.100 & 0.112 \\
\hline & 0.176 & 4.657 & 1.867 & 0.144 & 4.458 & 1.042 \\
\hline \multirow[t]{2}{*}{$\mathrm{O}$} & -0.339 & -0.033 & 0.524 & -0.865 & -0.691 & 0.246 \\
\hline & 1.190 & 0.171 & 3.493 & 4.951 & 1.722 & 2.014 \\
\hline \multirow[t]{2}{*}{$\mathrm{P}$} & -1.822 & -0.367 & 0.411 & 0.234 & 0.939 & -1.019 \\
\hline & 3.167 & 1.381 & 1.301 & 0.731 & 1.816 & 2.967 \\
\hline \multirow[t]{2}{*}{$\mathrm{Y}$} & 0.685 & 1.311 & 1.813 & 0.750 & 1.158 & 1.625 \\
\hline & 12.284 & 7.279 & 14.467 & 7.300 & 10.581 & 5.475 \\
\hline & \multicolumn{6}{|c|}{ REN1 } \\
\hline Equation & $\mathrm{F}$ & A & C & $\mathrm{G}$ & $\mathrm{O}$ & $\mathrm{P}$ \\
\hline \multirow[t]{2}{*}{$\mathrm{F}$} & -0.971 & 0.023 & 0.162 & -0.214 & 0.196 & 0.209 \\
\hline & 7.122 & 0.342 & 2.445 & 2.905 & 1.722 & 3.643 \\
\hline \multirow[t]{2}{*}{ A } & -0.434 & -1.905 & -0.132 & 0.922 & 0.736 & -1.147 \\
\hline & 0.539 & 2.535 & 0.339 & 2.109 & 0.799 & 3.566 \\
\hline \multirow[t]{2}{*}{ C } & 0.163 & -0.022 & -1.277 & 0.163 & -0.269 & -0.219 \\
\hline & 0.716 & 0.182 & 6.431 & 1.293 & 1.349 & 2.242 \\
\hline \multirow[t]{2}{*}{ G } & -0.867 & 0.325 & 0.236 & -0.598 & 0.118 & -0.051 \\
\hline & 3.328 & 2.459 & 1.938 & 3.568 & 0.562 & 0.516 \\
\hline \multirow[t]{2}{*}{$\mathrm{O}$} & 0.126 & 0.224 & -0.248 & 0.010 & -1.910 & 0.219 \\
\hline & 0.343 & 0.868 & 1.427 & 0.050 & 4.133 & 1.355 \\
\hline \multirow[t]{2}{*}{$\mathrm{P}$} & 2.695 & -1.588 & -1.059 & -0.376 & 0.934 & -2.681 \\
\hline & 4.011 & 3.752 & 2.803 & 0.878 & 1.264 & 6.300 \\
\hline \multirow[t]{2}{*}{ Y } & 0.596 & 1.960 & 1.462 & 0.837 & 1.580 & 2.075 \\
\hline & 10.056 & 6.408 & 8.920 & 5.888 & 9.114 & 4.480 \\
\hline & \multicolumn{6}{|c|}{ REN2 } \\
\hline Equation & F & A & C & G & $\mathrm{O}$ & $\mathrm{P}$ \\
\hline \multirow[t]{2}{*}{ F } & -0.220 & -0.135 & -0.035 & -0.143 & -0.002 & -0.138 \\
\hline & 0.897 & 1.045 & 0.331 & 1.646 & 0.009 & 1.596 \\
\hline \multirow[t]{2}{*}{ A } & -1.535 & -2.097 & 1.733 & 1.215 & 0.504 & -0.039 \\
\hline & 0.953 & 1.398 & 2.328 & 1.844 & 0.340 & 0.060 \\
\hline \multirow[t]{2}{*}{ C } & -0.415 & 0.484 & -1.584 & 0.024 & 0.219 & 0.036 \\
\hline & 1.053 & 2.133 & 4.737 & 0.121 & 0.676 & 0.205 \\
\hline \multirow[t]{2}{*}{ G } & -0.758 & 0.376 & 0.065 & -0.125 & -0.517 & 0.018 \\
\hline & 2.112 & 1.665 & 0.293 & 0.494 & 1.472 & 0.111 \\
\hline $\mathrm{O}$ & -0.717 & 0.068 & 0.130 & -0.585 & -1.075 & 0.191 \\
\hline & 1.113 & 0.156 & 0.414 & 1.941 & 1.334 & 0.681 \\
\hline P & -3.515 & -0.160 & 0.028 & -0.115 & 0.849 & 0.420 \\
\hline & 2.436 & 0.174 & 0.034 & 0.173 & 0.648 & 0.470 \\
\hline Y & 0.673 & 0.217 & 1.236 & 0.941 & 1.988 & 2.494 \\
\hline & 8.376 & 0.372 & 5.139 & 4.180 & 8.158 & 3.499 \\
\hline
\end{tabular}


Table 25: FACGOP System Elasticity Estimates

REN0, REN1 and REN2 Households.

Estimates Based on Three-Region Data, 1980-1992.

\begin{tabular}{|c|c|c|c|c|c|c|}
\hline \multirow[b]{2}{*}{ Equation } & \multicolumn{6}{|c|}{$\overline{\text { REN0 }}$} \\
\hline & $\mathrm{F}$ & A & $\mathrm{C}$ & G & $\mathrm{O}$ & $\mathrm{P}$ \\
\hline \multirow[t]{2}{*}{$\mathrm{F}$} & -0.653 & 0.047 & 0.030 & -0.026 & -0.035 & -0.122 \\
\hline & 3.772 & 0.604 & 0.332 & 0.359 & 0.270 & 1.493 \\
\hline \multirow[t]{2}{*}{ A } & 0.273 & -2.143 & -0.222 & 1.228 & -0.222 & 0.036 \\
\hline & 0.415 & 3.402 & 0.660 & 3.444 & 0.290 & 0.149 \\
\hline \multirow[t]{2}{*}{$\mathrm{C}$} & -0.336 & -0.124 & -1.371 & -0.653 & 0.700 & 0.062 \\
\hline & 1.091 & 0.919 & 4.883 & 3.848 & 2.563 & 0.289 \\
\hline \multirow[t]{2}{*}{ G } & -0.108 & 0.523 & -0.553 & 0.420 & -0.965 & -0.117 \\
\hline & 0.407 & 3.423 & 3.089 & 2.240 & 3.428 & 0.921 \\
\hline \multirow[t]{2}{*}{$\mathrm{O}$} & -0.265 & -0.069 & 0.604 & -0.732 & -0.840 & 0.186 \\
\hline & 0.800 & 0.306 & 2.921 & 3.751 & 1.744 & 1.211 \\
\hline \multirow[t]{2}{*}{$\mathrm{P}$} & -1.559 & 0.025 & 0.217 & -0.413 & 0.698 & -0.375 \\
\hline & 2.538 & 0.085 & 0.542 & 1.264 & 1.133 & 0.926 \\
\hline \multirow[t]{2}{*}{ Y } & 0.759 & 1.049 & 1.722 & 0.800 & 1.115 & 1.408 \\
\hline & 18.033 & 7.176 & 15.148 & 9.022 & 11.638 & 5.497 \\
\hline & \multicolumn{6}{|c|}{ REN1 } \\
\hline Equation & $\mathrm{F}$ & A & C & $\mathrm{G}$ & $\mathrm{O}$ & $\mathrm{P}$ \\
\hline \multirow[t]{2}{*}{$\mathrm{F}$} & -0.981 & 0.022 & 0.062 & -0.125 & 0.014 & 0.212 \\
\hline & 6.834 & 0.402 & 0.892 & 2.285 & 0.123 & 4.325 \\
\hline \multirow[t]{2}{*}{ A } & -0.437 & -1.647 & 0.456 & 1.033 & -0.817 & -0.800 \\
\hline & 0.636 & 2.600 & 1.308 & 3.003 & 0.848 & 2.700 \\
\hline \multirow[t]{2}{*}{ C } & -0.405 & 0.144 & -1.538 & -0.242 & 0.105 & -0.157 \\
\hline & 1.533 & 1.329 & 6.054 & 1.879 & 0.442 & 1.350 \\
\hline \multirow[t]{2}{*}{ G } & -0.101 & 0.398 & 0.027 & -0.091 & -0.090 & -0.220 \\
\hline & 0.530 & 3.903 & 0.222 & 0.644 & 0.456 & 2.739 \\
\hline \multirow[t]{2}{*}{$\mathrm{O}$} & -0.092 & -0.166 & 0.273 & -0.245 & -0.780 & -0.017 \\
\hline & 0.233 & 0.617 & 1.297 & 1.331 & 1.528 & 0.104 \\
\hline \multirow[t]{2}{*}{$\mathrm{P}$} & 2.985 & -1.105 & -0.740 & -1.189 & -0.256 & -1.640 \\
\hline & 3.630 & 2.735 & 1.505 & 3.326 & 0.323 & 3.303 \\
\hline \multirow[t]{3}{*}{ Y } & 0.796 & 2.213 & 2.093 & 0.079 & 1.027 & 1.944 \\
\hline & 13.319 & 7.644 & 12.099 & 0.402 & 4.525 & 3.532 \\
\hline & \multicolumn{6}{|c|}{ REN2 } \\
\hline Equation & $\mathrm{F}$ & A & $\mathrm{C}$ & G & $\mathrm{O}$ & $\mathrm{P}$ \\
\hline \multirow[t]{2}{*}{$\mathrm{F}$} & 0.015 & 0.071 & -0.255 & -0.292 & -0.405 & -0.137 \\
\hline & 0.045 & 0.433 & 1.658 & 2.460 & 1.579 & 1.256 \\
\hline \multirow[t]{2}{*}{ A } & 0.877 & -2.919 & 0.931 & 0.220 & -0.550 & 0.216 \\
\hline & 0.381 & 1.293 & 0.739 & 0.206 & 0.227 & 0.220 \\
\hline \multirow[t]{2}{*}{$\mathrm{C}$} & -0.969 & 0.254 & -0.679 & -0.224 & 0.347 & 0.236 \\
\hline & 1.692 & 0.760 & 1.541 & 0.817 & 0.702 & 0.947 \\
\hline \multirow[t]{2}{*}{ G } & -1.090 & 0.105 & -0.202 & 0.151 & 0.843 & -0.298 \\
\hline & 1.988 & 0.298 & 0.600 & 0.279 & 1.458 & 0.469 \\
\hline $\mathrm{O}$ & -1.783 & -0.162 & 0.334 & 0.631 & -0.513 & 0.191 \\
\hline & 1.718 & 0.235 & 0.625 & 1.257 & 0.402 & 0.366 \\
\hline P & -2.303 & 0.256 & 1.032 & -1.110 & 0.804 & 0.230 \\
\hline & 1.296 & 0.231 & 0.950 & 1.134 & 0.498 & 0.199 \\
\hline Y & 1.003 & 1.224 & 1.035 & 0.492 & 1.303 & 1.090 \\
\hline & 25.150 & 3.183 & 9.444 & 3.060 & 9.338 & 2.916 \\
\hline
\end{tabular}


Table 26: FAC System Elasticity Estimates

MOR0, MOR1 and MOR2 Households.

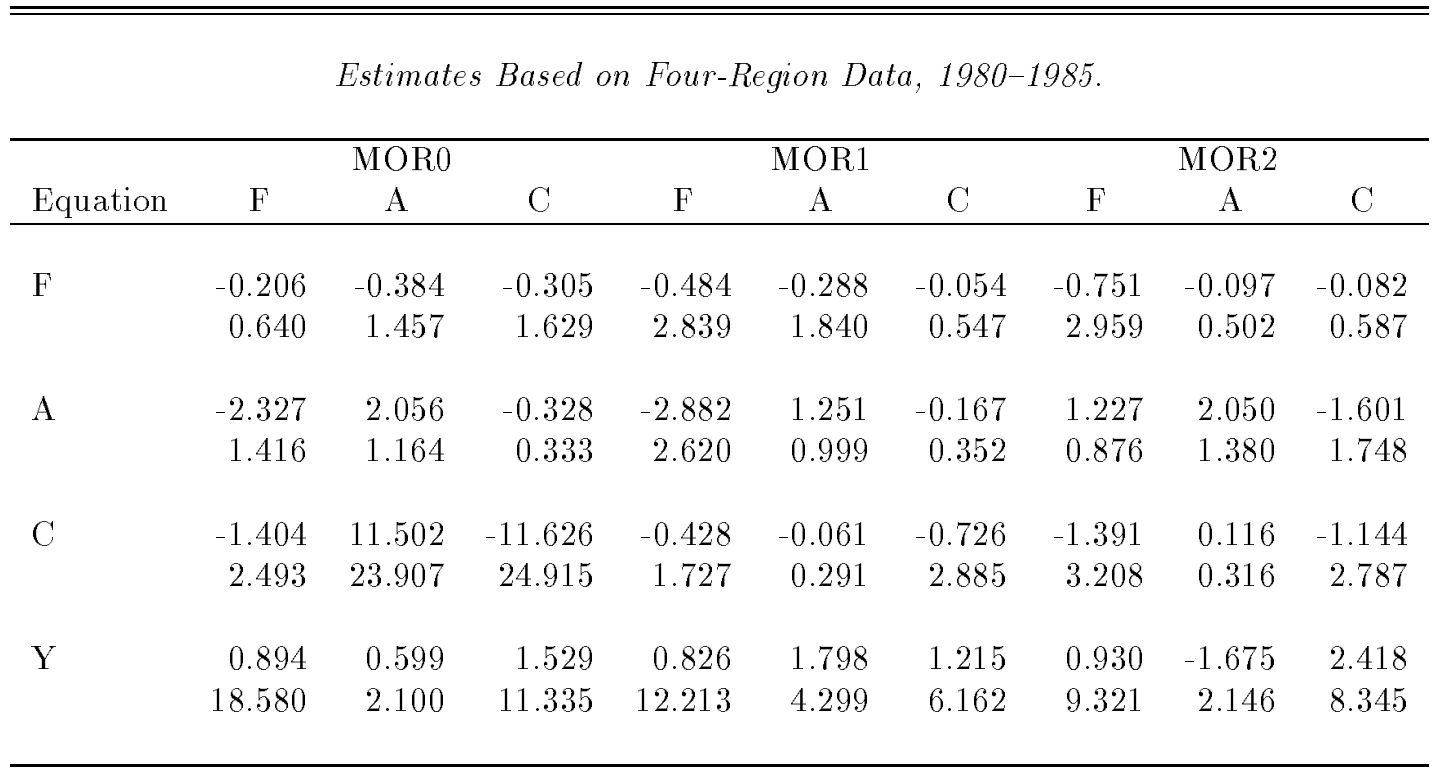

Estimates Based on Four-Region Data, 1980-1992.

\begin{tabular}{lccccccccc}
\hline & & \multicolumn{3}{c}{ MOR0 } & & \multicolumn{3}{c}{ MOR1 } & \multicolumn{3}{c}{ MOR2 } \\
Equation & $\mathrm{F}$ & $\mathrm{A}$ & $\mathrm{C}$ & $\mathrm{F}$ & $\mathrm{A}$ & $\mathrm{C}$ & $\mathrm{F}$ & \multicolumn{1}{c}{$\mathrm{A}$} & $\mathrm{C}$ \\
\hline $\mathrm{F}$ & & & & & & & & & \\
& -1.059 & 0.219 & 0.186 & -0.895 & 0.066 & -0.004 & -0.434 & -0.052 & -0.184 \\
& 7.788 & 1.980 & 2.477 & 5.611 & 0.536 & 0.045 & 1.713 & 0.406 & 1.077 \\
$\mathrm{~A}$ & & & & & & & & & \\
& 1.055 & -3.318 & 0.023 & 0.594 & -1.397 & -0.176 & -2.063 & -1.697 & 1.003 \\
& 0.975 & 3.023 & 0.040 & 0.493 & 1.279 & 0.382 & 1.489 & 1.831 & 1.268 \\
$\mathrm{C}$ & & & & & & & & & \\
& -0.168 & -2.280 & 0.769 & -0.480 & 0.099 & -1.133 & -1.274 & -0.054 & -0.273 \\
& 0.741 & 11.600 & 3.173 & 2.362 & 0.792 & 6.634 & 2.466 & 0.274 & 0.733 \\
$\mathrm{Y}$ & & & & & & & & & \\
& 0.654 & 2.240 & 1.679 & 0.833 & 0.980 & 1.514 & 0.669 & 2.758 & 1.601 \\
& 13.408 & 6.890 & 13.581 & 12.820 & 2.102 & 9.674 & 10.614 & 5.327 & 9.366 \\
& & & & & & & & & \\
\hline
\end{tabular}

Estimates Based on Three-Region Data, 1980-1992.

\begin{tabular}{lccccccccc}
\hline & & \multicolumn{1}{c}{ MOR0 } & & \multicolumn{3}{c}{ MOR1 } & \multicolumn{3}{c}{ MOR2 } \\
Equation & $\mathrm{F}$ & $\mathrm{A}$ & $\mathrm{C}$ & $\mathrm{F}$ & $\mathrm{A}$ & $\mathrm{C}$ & $\mathrm{F}$ & $\mathrm{A}$ & $\mathrm{C}$ \\
\hline & & & & & & & & & \\
$\mathrm{F}$ & -0.930 & 0.126 & 0.070 & -0.302 & -0.047 & -0.655 & -0.817 & 0.077 & 0.074 \\
& 8.250 & 1.139 & 0.896 & 0.814 & 0.212 & 3.078 & 7.923 & 0.993 & 1.092 \\
$\mathrm{~A}$ & & & & & & & & & \\
& 0.664 & -3.367 & 1.282 & 0.080 & -0.073 & -0.090 & -0.816 & -1.799 & -0.637 \\
& 0.707 & 3.099 & 1.756 & 0.036 & 0.041 & 0.086 & 0.874 & 1.953 & 1.011 \\
$\mathrm{C}$ & & & & & & & & & \\
& -0.433 & 4.537 & -5.774 & -2.075 & 7.003 & -6.161 & -0.375 & -0.275 & -0.847 \\
& 2.125 & 18.895 & 20.576 & 3.428 & 24.949 & 11.748 & 1.751 & 1.780 & 4.090 \\
$\mathrm{Y}$ & & & & & & & & & \\
& 0.735 & 1.421 & 1.670 & 1.005 & 0.084 & 1.233 & 0.666 & 3.251 & 1.497 \\
& 17.767 & 4.163 & 13.775 & 14.946 & 0.193 & 9.553 & 10.721 & 6.754 & 9.466 \\
& & & & & & & & & \\
\hline
\end{tabular}


Table 27: FAC System Elasticity Estimates

REN0, REN1 and REN2 Households.

\begin{tabular}{|c|c|c|c|c|c|c|c|c|c|}
\hline \multirow[b]{3}{*}{ Equation } & \multicolumn{9}{|c|}{ Estimates Based on Four-Region Data, 1980-1985. } \\
\hline & \multicolumn{3}{|c|}{$\begin{array}{l}\text { REN0 } \\
\end{array}$} & \multicolumn{3}{|c|}{$\overline{\text { REN1 }}$} & \multicolumn{3}{|c|}{$\begin{array}{l}\text { REN2 } \\
\end{array}$} \\
\hline & $\mathrm{F}$ & A & $\mathrm{C}$ & $\mathrm{F}$ & A & $\mathrm{C}$ & $\mathrm{F}$ & A & $\mathrm{C}$ \\
\hline \multirow[t]{2}{*}{$\mathrm{F}$} & -1.134 & -0.070 & 0.122 & 0.244 & -1.225 & 0.024 & -1.295 & 0.080 & 0.101 \\
\hline & 4.772 & 0.355 & 0.907 & 0.492 & 2.598 & 0.166 & 3.228 & 0.244 & 0.581 \\
\hline \multirow[t]{2}{*}{ A } & -0.367 & -0.515 & -0.130 & -9.474 & 9.647 & -1.213 & 1.507 & -0.941 & -0.488 \\
\hline & 0.320 & 0.457 & 0.249 & 2.584 & 2.532 & 1.054 & 0.605 & 0.403 & 0.442 \\
\hline \multirow[t]{2}{*}{ C } & 0.641 & 0.030 & -1.398 & -0.047 & 0.013 & -1.108 & 0.451 & -0.536 & -0.998 \\
\hline & 1.868 & 0.115 & 4.435 & 0.088 & 0.024 & 3.070 & 0.645 & 0.949 & 2.015 \\
\hline \multirow[t]{2}{*}{ Y } & 1.081 & 1.011 & 0.727 & 0.957 & 1.040 & 1.142 & 1.114 & -0.079 & 1.083 \\
\hline & 12.906 & 2.692 & 2.749 & 24.506 & 3.409 & 14.529 & 13.995 & 0.133 & 7.229 \\
\hline
\end{tabular}

Estimates Based on Four-Region Data, 1980-1992.

\begin{tabular}{|c|c|c|c|c|c|c|c|c|c|}
\hline \multirow[b]{2}{*}{ Equation } & \multicolumn{3}{|c|}{$\overline{\text { REN0 }}$} & \multicolumn{3}{|c|}{$\overline{\text { REN1 }}$} & \multicolumn{3}{|c|}{$\overline{\mathrm{REN} 2}$} \\
\hline & $\mathrm{F}$ & A & C & $\mathrm{F}$ & A & C & $\mathrm{F}$ & A & $\mathrm{C}$ \\
\hline \multirow[t]{2}{*}{ F } & -0.934 & 0.108 & 0.043 & -0.776 & 0.039 & 0.072 & -1.019 & 0.014 & 0.029 \\
\hline & 4.384 & 0.624 & 0.415 & 5.533 & 0.422 & 0.798 & 5.152 & 0.100 & 0.277 \\
\hline \multirow[t]{2}{*}{ A } & 0.475 & -2.490 & 0.683 & -0.537 & -2.031 & 0.533 & 0.127 & -3.047 & 1.872 \\
\hline & 0.346 & 1.927 & 1.191 & 0.536 & 2.240 & 1.024 & 0.069 & 1.504 & 2.259 \\
\hline \multirow[t]{2}{*}{ C } & -0.423 & 1.438 & -2.626 & -0.646 & -0.208 & -1.041 & 0.036 & 0.767 & -1.884 \\
\hline & 1.238 & 6.079 & 9.800 & 2.455 & 1.305 & 4.535 & 0.086 & 3.131 & 4.447 \\
\hline \multirow[t]{2}{*}{ Y } & 0.783 & 1.331 & 1.612 & 0.665 & 2.034 & 1.895 & 0.976 & 1.048 & 1.081 \\
\hline & 17.272 & 5.377 & 12.666 & 8.422 & 3.681 & 8.939 & 62.861 & 11.053 & 16.999 \\
\hline
\end{tabular}

Estimates Based on Three-Region Data, 1980-1992.

\begin{tabular}{|c|c|c|c|c|c|c|c|c|c|}
\hline \multirow[b]{2}{*}{ Equation } & \multicolumn{3}{|c|}{$\begin{array}{l}\text { REN0 } \\
\end{array}$} & \multicolumn{3}{|c|}{ REN1 } & \multicolumn{3}{|c|}{$\begin{array}{l}\text { REN2 } \\
\end{array}$} \\
\hline & $\mathrm{F}$ & A & C & $\mathrm{F}$ & A & C & $\mathrm{F}$ & A & C \\
\hline \multirow[t]{2}{*}{$\mathrm{F}$} & -0.903 & 0.148 & 0.040 & -0.484 & -0.115 & -0.125 & -2.283 & 1.020 & 0.295 \\
\hline & 5.010 & 1.471 & 0.294 & 1.692 & 0.770 & 0.741 & 5.287 & 3.032 & 1.624 \\
\hline \multirow[t]{2}{*}{ A } & 1.056 & -2.306 & 0.181 & -2.429 & -1.124 & 1.436 & 14.725 & -14.221 & -1.281 \\
\hline & 1.249 & 3.154 & 0.399 & 1.402 & 0.972 & 1.424 & 3.094 & 3.533 & 0.645 \\
\hline \multirow[t]{2}{*}{$\mathrm{C}$} & -0.738 & -0.098 & -1.102 & -1.192 & -3.277 & 2.775 & 0.946 & 10.045 & -12.168 \\
\hline & 1.939 & 0.560 & 3.048 & 2.064 & 11.367 & 6.731 & 1.439 & 19.648 & 25.093 \\
\hline \multirow[t]{2}{*}{ Y } & 0.715 & 1.069 & 1.937 & 0.723 & 2.117 & 1.694 & 0.968 & 0.777 & 1.177 \\
\hline & 11.633 & 4.071 & 9.706 & 9.753 & 4.516 & 7.936 & 29.411 & 1.495 & 11.499 \\
\hline
\end{tabular}


Table 28: GOP System Elasticity Estimates

MOR0, MOR1 and MOR2 Households.

Estimates Based on Four-Region Data, 1980-1985.

\begin{tabular}{|c|c|c|c|c|c|c|c|c|c|}
\hline \multirow[b]{2}{*}{ Equation } & \multicolumn{3}{|c|}{ MOR0 } & \multicolumn{3}{|c|}{ MOR1 } & \multicolumn{3}{|c|}{ MOR2 } \\
\hline & $\mathrm{G}$ & $\mathrm{O}$ & $\mathrm{P}$ & $\mathrm{G}$ & $\mathrm{O}$ & $\mathrm{P}$ & $\mathrm{G}$ & $\mathrm{O}$ & $\mathrm{P}$ \\
\hline \multirow[t]{2}{*}{$\mathrm{G}$} & -0.044 & -0.472 & -0.438 & 0.099 & -0.491 & -0.215 & 0.568 & -0.526 & -1.050 \\
\hline & 0.111 & 0.918 & 1.301 & 0.266 & 1.585 & 1.274 & 1.027 & 1.003 & 3.304 \\
\hline \multirow[t]{2}{*}{$\mathrm{O}$} & -0.486 & -0.007 & -0.456 & -0.818 & -0.736 & 0.243 & -0.596 & -0.162 & -0.262 \\
\hline & 0.924 & 0.009 & 0.992 & 2.416 & 2.381 & 1.509 & 1.035 & 0.254 & 0.668 \\
\hline \multirow[t]{2}{*}{$\mathrm{P}$} & -1.621 & -0.361 & 0.660 & -1.139 & -0.475 & 0.282 & -5.555 & -1.041 & 5.734 \\
\hline & 1.455 & 0.237 & 0.443 & 1.445 & 0.808 & 0.480 & 3.258 & 0.549 & 2.798 \\
\hline \multirow[t]{2}{*}{$\mathrm{Y}$} & 0.954 & 0.948 & 1.322 & 0.607 & 1.311 & 1.332 & 1.008 & 1.020 & 0.862 \\
\hline & 14.237 & 13.034 & 7.831 & 3.698 & 9.377 & 2.654 & 14.795 & 14.979 & 4.372 \\
\hline
\end{tabular}

Estimates Based on Four-Region Data, 1980-1992.

\begin{tabular}{|c|c|c|c|c|c|c|c|c|c|}
\hline \multirow[b]{2}{*}{ Equation } & \multicolumn{3}{|c|}{ MOR0 } & \multicolumn{3}{|c|}{ MOR1 } & \multicolumn{3}{|c|}{ MOR2 } \\
\hline & $\mathrm{G}$ & $\mathrm{O}$ & $\mathrm{P}$ & $\mathrm{G}$ & $\mathrm{O}$ & $\mathrm{P}$ & $\mathrm{G}$ & $\mathrm{O}$ & $\mathrm{P}$ \\
\hline \multirow[t]{2}{*}{$\mathrm{G}$} & -0.074 & -0.551 & 0.305 & -0.797 & -0.208 & 0.090 & 0.676 & -1.759 & 0.251 \\
\hline & 0.237 & 1.411 & 2.090 & 4.246 & 1.292 & 0.708 & 1.627 & 3.053 & 0.902 \\
\hline \multirow[t]{2}{*}{$\mathrm{O}$} & -0.939 & -0.369 & -0.160 & -0.315 & -1.082 & 0.085 & -1.676 & 1.399 & -0.928 \\
\hline & 2.934 & 0.875 & 1.027 & 1.977 & 5.251 & 0.755 & 3.382 & 1.786 & 2.072 \\
\hline \multirow[t]{2}{*}{$\mathrm{P}$} & 0.773 & -0.548 & -1.631 & 0.927 & 0.340 & -0.782 & 1.543 & -132.664 & 130.529 \\
\hline & 1.575 & 0.781 & 4.412 & 1.574 & 0.613 & 1.377 & 0.986 & 44.879 & 61.972 \\
\hline \multirow[t]{2}{*}{$Y$} & 0.321 & 1.468 & 1.407 & 0.915 & 1.312 & -0.485 & 0.833 & 1.206 & 0.592 \\
\hline & 2.460 & 11.046 & 3.973 & 6.107 & 11.069 & 0.796 & 13.164 & 16.130 & 3.487 \\
\hline
\end{tabular}

Estimates Based on Three-Region Data, 1980-1992.

\begin{tabular}{|c|c|c|c|c|c|c|c|c|c|}
\hline \multirow[b]{2}{*}{ Equation } & \multicolumn{3}{|c|}{ MOR0 } & \multicolumn{3}{|c|}{ MOR1 } & \multicolumn{3}{|c|}{ MOR2 } \\
\hline & $\mathrm{G}$ & $\mathrm{O}$ & $\mathrm{P}$ & G & $\mathrm{O}$ & $\mathrm{P}$ & $\mathrm{G}$ & $\mathrm{O}$ & $\mathrm{P}$ \\
\hline \multirow[t]{2}{*}{$\mathrm{G}$} & -0.034 & -0.767 & 0.353 & -0.908 & 0.003 & -0.012 & 0.291 & -1.511 & 0.351 \\
\hline & 0.107 & 1.736 & 1.962 & 2.742 & 0.010 & 0.078 & 0.840 & 2.743 & 1.260 \\
\hline \multirow[t]{2}{*}{$\mathrm{O}$} & -0.993 & -0.194 & -0.137 & -0.123 & -1.225 & 0.132 & -1.453 & 1.447 & -1.182 \\
\hline & 2.630 & 0.374 & 0.564 & 0.463 & 3.601 & 0.779 & 3.048 & 1.764 & 2.627 \\
\hline \multirow[t]{2}{*}{$\mathrm{P}$} & 0.851 & -17.205 & 14.750 & 0.284 & -1.136 & 0.745 & 2.220 & -137.233 & 134.515 \\
\hline & 1.329 & 15.329 & 16.567 & 0.350 & 1.213 & 1.296 & 1.369 & 45.547 & 65.376 \\
\hline \multirow[t]{2}{*}{ Y } & 0.448 & 1.324 & 1.603 & 0.917 & 1.216 & 0.106 & 0.869 & 1.189 & 0.497 \\
\hline & 5.485 & 17.620 & 5.753 & 9.587 & 15.276 & 0.256 & 14.367 & 14.242 & 2.108 \\
\hline
\end{tabular}


Table 29: GOP System Elasticity Estimates

REN0, REN1 and REN2 Households.

\begin{tabular}{|c|c|c|c|c|c|c|c|c|c|}
\hline \multirow[b]{3}{*}{ Equation } & \multicolumn{9}{|c|}{ Estimates Based on Four-Region Data, 1980-1985. } \\
\hline & \multicolumn{3}{|c|}{ REN0 } & \multicolumn{3}{|c|}{$\overline{\text { REN1 }}$} & \multicolumn{3}{|c|}{$\overline{\text { REN2 }}$} \\
\hline & G & $\mathrm{O}$ & $\mathrm{P}$ & G & $\mathrm{O}$ & $\mathrm{P}$ & G & $\mathrm{O}$ & $\mathrm{P}$ \\
\hline \multirow[t]{2}{*}{ G } & 0.380 & -0.813 & -0.009 & -0.689 & 0.309 & -0.188 & 0.313 & -0.542 & -0.745 \\
\hline & 0.936 & 2.206 & 0.042 & 1.873 & 0.922 & 0.966 & 0.526 & 1.250 & 1.600 \\
\hline \multirow[t]{2}{*}{$\mathrm{O}$} & -1.296 & -0.194 & -0.075 & -0.014 & -1.620 & 0.322 & -0.821 & 0.136 & -0.626 \\
\hline & 3.749 & 0.463 & 0.409 & 0.032 & 2.955 & 1.262 & 1.609 & 0.238 & 1.361 \\
\hline \multirow[t]{2}{*}{$\mathrm{P}$} & -0.149 & 0.027 & -0.830 & -0.868 & -0.234 & -0.463 & -1.868 & -9.631 & 11.203 \\
\hline & 0.302 & 0.058 & 1.844 & 1.541 & 0.462 & 0.955 & 1.367 & 8.665 & 7.479 \\
\hline \multirow[t]{2}{*}{ Y } & 0.442 & 1.566 & 0.952 & 0.568 & 1.312 & 1.565 & 0.974 & 1.312 & 0.295 \\
\hline & 1.963 & 8.723 & 2.169 & 4.199 & 7.490 & 4.384 & 14.136 & 13.876 & 1.495 \\
\hline
\end{tabular}

Estimates Based on Four-Region Data, 1980-1992.

\begin{tabular}{lccccccccc}
\hline & & REN0 & & \multicolumn{3}{c}{ REN1 } & \multicolumn{3}{c}{ REN2 } \\
Equation & $\mathrm{G}$ & $\mathrm{O}$ & $\mathrm{P}$ & $\mathrm{G}$ & $\mathrm{O}$ & $\mathrm{P}$ & $\mathrm{G}$ & $\mathrm{O}$ & $\mathrm{P}$ \\
\hline $\mathrm{G}$ & & & & & & & & & \\
& -0.915 & -0.387 & 0.533 & -0.815 & 0.005 & 0.092 & 0.194 & -0.357 & -0.494 \\
& 3.063 & 1.916 & 2.144 & 4.358 & 0.028 & 0.536 & 0.572 & 0.911 & 1.750 \\
$\mathrm{O}$ & & & & & & & & & \\
& -0.508 & -0.793 & 0.123 & -0.259 & -1.560 & 0.552 & -0.694 & -0.341 & -0.306 \\
& 2.593 & 3.916 & 0.691 & 1.397 & 5.131 & 2.452 & 1.762 & 0.561 & 0.872 \\
$\mathrm{P}$ & & & & & & & & & \\
& 1.402 & -0.071 & -2.392 & 0.210 & 0.278 & -1.607 & -1.964 & -1.527 & 2.424 \\
& 2.030 & 0.118 & 3.012 & 0.355 & 0.370 & 1.704 & 1.978 & 1.242 & 2.265 \\
$\mathrm{Y}$ & & & & & & & & & \\
& 0.769 & 1.178 & 1.060 & 0.718 & 1.266 & 1.119 & 0.657 & 1.342 & 1.066 \\
& 4.905 & 11.559 & 2.886 & 4.991 & 8.320 & 2.149 & 8.567 & 15.928 & 4.396 \\
& & & & & & & & & \\
\hline
\end{tabular}

Estimates Based on Three-Region Data, 1980-1992.

\begin{tabular}{lccccccccc}
\hline & & REN0 & & \multicolumn{3}{c}{ REN1 } & & \multicolumn{3}{c}{ REN2 } \\
Equation & $\mathrm{G}$ & $\mathrm{O}$ & $\mathrm{P}$ & $\mathrm{G}$ & $\mathrm{O}$ & $\mathrm{P}$ & $\mathrm{G}$ & $\mathrm{O}$ & $\mathrm{P}$ \\
\hline \multirow{3}{*}{$\mathrm{G}$} & & & & & & & & & \\
& -0.643 & -0.617 & 0.553 & 0.135 & -0.440 & -0.374 & -0.551 & -0.541 & 0.351 \\
& 2.411 & 2.715 & 2.116 & 0.381 & 1.472 & 2.312 & 2.141 & 1.709 & 1.772 \\
$\mathrm{O}$ & & & & & & & & & \\
& -0.742 & -0.595 & 0.083 & -0.885 & -1.035 & 0.345 & -0.815 & -0.030 & -0.467 \\
& 3.625 & 2.120 & 0.425 & 2.865 & 2.659 & 1.803 & 2.634 & 0.061 & 1.775 \\
$\mathrm{P}$ & & & & & & & & & \\
& 1.351 & 0.020 & -2.329 & -1.074 & 1.775 & -0.958 & 1.115 & 0.088 & -2.039 \\
& 2.054 & 0.031 & 2.750 & 2.171 & 3.008 & 1.689 & 1.970 & 0.109 & 3.142 \\
$\mathrm{Y}$ & & & & & & & & & \\
& 0.707 & 1.254 & 0.958 & 0.679 & 1.575 & 0.257 & 0.741 & 1.312 & 0.836 \\
& 4.718 & 11.074 & 2.151 & 7.627 & 14.583 & 1.028 & 6.495 & 11.923 & 2.769 \\
& & & & & & & & & \\
\hline
\end{tabular}


Table 30: HES System Elasticity Estimates

MOR0, MOR1 and MOR2 Households.

Estimates Based on Four-Region Data, 1980-1985.

\begin{tabular}{|c|c|c|c|c|c|c|c|c|c|}
\hline \multirow[b]{2}{*}{ Equation } & \multicolumn{3}{|c|}{ MOR0 } & \multicolumn{3}{|c|}{ MOR1 } & \multicolumn{3}{|c|}{ MOR2 } \\
\hline & $\mathrm{H}$ & $\mathrm{E}$ & $\mathrm{S}$ & $\mathrm{H}$ & $\mathrm{E}$ & $\mathrm{S}$ & $\mathrm{H}$ & $\mathrm{E}$ & $\mathrm{S}$ \\
\hline \multirow[t]{2}{*}{$\mathrm{H}$} & -1.608 & 3.249 & 2.523 & -2.048 & 0.164 & 0.539 & -1.445 & -4.105 & -3.264 \\
\hline & 1.197 & 1.032 & 0.588 & 0.183 & 0.128 & 0.043 & 1.424 & 5.293 & 2.225 \\
\hline \multirow[t]{2}{*}{$\mathrm{E}$} & 0.091 & -2.148 & 1.098 & 0.270 & -1.449 & 0.250 & 0.283 & -1.801 & 0.647 \\
\hline & 0.036 & 1.828 & 0.402 & 0.021 & 4.596 & 0.020 & 0.130 & 4.458 & 0.278 \\
\hline \multirow[t]{2}{*}{$\mathrm{S}$} & 0.169 & 0.828 & -4.104 & 1.119 & 0.550 & -2.261 & -0.009 & 5.823 & 1.672 \\
\hline & 0.053 & 0.550 & 1.843 & 0.076 & 0.262 & 0.177 & 0.002 & 4.955 & 0.477 \\
\hline \multirow[t]{2}{*}{$\mathrm{Y}$} & -4.164 & 0.959 & 3.107 & 1.346 & 0.929 & 0.591 & 8.814 & 0.870 & -7.486 \\
\hline & 0.290 & 1.921 & 0.542 & 0.036 & 4.753 & 0.010 & 1.103 & 6.071 & 0.837 \\
\hline
\end{tabular}

Estimates Based on Four-Region Data, 1980-1992.

\begin{tabular}{|c|c|c|c|c|c|c|c|c|c|}
\hline \multirow[b]{2}{*}{ Equation } & \multicolumn{3}{|c|}{ MOR0 } & \multicolumn{3}{|c|}{ MOR1 } & \multicolumn{3}{|c|}{ MOR2 } \\
\hline & $\mathrm{H}$ & $\mathrm{E}$ & $\mathrm{S}$ & $\mathrm{H}$ & $\mathrm{E}$ & $\mathrm{S}$ & $\mathrm{H}$ & $\mathrm{E}$ & $\mathrm{S}$ \\
\hline \multirow[t]{2}{*}{$\mathrm{H}$} & -0.256 & 1.156 & 0.530 & -52.491 & -388.859 & -130.096 & 0.597 & -1.327 & -0.796 \\
\hline & 0.213 & 0.574 & 0.170 & 0.050 & 0.174 & 0.040 & 0.905 & 3.229 & 1.826 \\
\hline \multirow[t]{2}{*}{$\mathrm{E}$} & -0.168 & -0.433 & -0.263 & 0.013 & -0.867 & 0.329 & -0.684 & -0.849 & 0.255 \\
\hline & 0.116 & 1.207 & 0.173 & 0.000 & 4.552 & 0.000 & 2.289 & 2.808 & 1.450 \\
\hline \multirow[t]{2}{*}{$\mathrm{S}$} & -0.099 & 0.585 & -2.766 & 71.784 & 541.633 & 180.061 & -0.577 & 1.038 & -0.229 \\
\hline & 0.060 & 0.539 & 2.797 & 0.010 & 0.173 & 0.046 & 0.790 & 2.679 & 0.279 \\
\hline \multirow[t]{2}{*}{$Y$} & -1.431 & 0.865 & 2.279 & 571.447 & 0.525 & -793.479 & 1.526 & 1.278 & -0.233 \\
\hline & 0.155 & 2.792 & 0.553 & 0.047 & 3.317 & 0.047 & 3.446 & 9.631 & 0.521 \\
\hline
\end{tabular}

Estimates Based on Three-Region Data, 1980-1992.

\begin{tabular}{lcrrrrrrrr}
\hline \multirow{2}{*}{ Equation } & $\mathrm{H}$ & $\mathrm{M}$ MOR0 & \multicolumn{1}{c}{$\mathrm{S}$} & \multicolumn{1}{c}{$\mathrm{H}$} & \multicolumn{1}{c}{$\mathrm{E}$} & $\mathrm{S}$ & $\mathrm{H}$ & $\mathrm{E}$ & $\mathrm{S}$ \\
\hline \multirow{3}{*}{$\mathrm{H}$} & & & & & & & & & \\
& -2.251 & 2.305 & 2.288 & -13.700 & -116.167 & -61.755 & 1.345 & 5.830 & 1.602 \\
& 2.838 & 1.809 & 1.583 & 0.190 & 0.704 & 0.261 & 0.471 & 5.106 & 0.417 \\
$\mathrm{E}$ & & & & & & & & & \\
& -0.105 & -0.722 & 0.092 & 0.029 & -0.812 & -0.016 & -0.036 & -0.842 & 0.035 \\
& 0.157 & 3.328 & 0.144 & 0.000 & 4.483 & 0.000 & 0.016 & 4.044 & 0.015 \\
$\mathrm{~S}$ & & & & & & & & & \\
& 0.861 & -0.084 & -4.045 & 17.083 & 155.881 & 82.991 & -2.734 & -6.073 & -4.303 \\
& 1.071 & 0.144 & 7.622 & 0.018 & 0.700 & 0.118 & 1.328 & 4.319 & 2.961 \\
$\mathrm{Y}$ & & & & & & & & & \\
& -2.342 & 0.735 & 3.268 & 191.622 & 0.800 & -255.956 & -8.776 & 0.843 & 13.110 \\
& 0.583 & 6.737 & 1.554 & 0.125 & 6.609 & 0.123 & 0.997 & 6.067 & 1.239 \\
& & & & & & & & & \\
\hline
\end{tabular}


Table 31: HES System Elasticity Estimates

REN0, REN1 and REN2 Households.

Estimates Based on Four-Region Data, 1980-1985.

\begin{tabular}{|c|c|c|c|c|c|c|c|c|c|}
\hline \multirow[b]{2}{*}{ Equation } & \multicolumn{3}{|c|}{ REN0 } & \multicolumn{3}{|c|}{ REN1 } & \multicolumn{3}{|c|}{ REN2 } \\
\hline & $\mathrm{H}$ & $\mathrm{E}$ & $\mathrm{S}$ & $\mathrm{H}$ & $\mathrm{E}$ & $\mathrm{S}$ & $\mathrm{H}$ & $\mathrm{E}$ & $\mathrm{S}$ \\
\hline \multirow[t]{2}{*}{$\mathrm{H}$} & -2.614 & -7.652 & -3.097 & -3.847 & -11.331 & -9.939 & -10.410 & -20.103 & -15.195 \\
\hline & 1.701 & 2.088 & 1.082 & 1.006 & 7.381 & 2.708 & 0.362 & 2.670 & 0.422 \\
\hline \multirow[t]{2}{*}{$\mathrm{E}$} & 0.134 & -1.137 & 0.150 & 0.138 & -1.077 & -0.223 & 0.759 & -2.459 & 0.883 \\
\hline & 0.034 & 2.845 & 0.036 & 0.022 & 2.979 & 0.035 & 0.016 & 6.726 & 0.018 \\
\hline \multirow[t]{2}{*}{$\mathrm{S}$} & 0.471 & 4.146 & -0.709 & 2.868 & 12.808 & 10.199 & 9.876 & 24.647 & 16.951 \\
\hline & 0.076 & 2.091 & 0.163 & 0.291 & 7.191 & 1.025 & 0.140 & 2.797 & 0.272 \\
\hline \multirow[t]{2}{*}{$\mathrm{Y}$} & 13.362 & 0.853 & -3.908 & 25.117 & 1.163 & -25.876 & 45.708 & 0.816 & -51.473 \\
\hline & 0.476 & 6.775 & 0.337 & 1.082 & 7.843 & 1.014 & 0.295 & 5.897 & 0.282 \\
\hline
\end{tabular}

Estimates Based on Four-Region Data, 1980-1992.

\begin{tabular}{|c|c|c|c|c|c|c|c|c|c|}
\hline \multirow[b]{2}{*}{ Equation } & \multicolumn{3}{|c|}{$\overline{\text { REN0 }}$} & \multicolumn{3}{|c|}{$\overline{R E N 1}$} & \multicolumn{3}{|c|}{$\overline{\text { REN2 }}$} \\
\hline & $\mathrm{H}$ & $\mathrm{E}$ & $\mathrm{S}$ & $\mathrm{H}$ & $\mathrm{E}$ & $\mathrm{S}$ & $\mathrm{H}$ & $\mathrm{E}$ & $\mathrm{S}$ \\
\hline \multirow[t]{2}{*}{$\mathrm{H}$} & 1.162 & -7.115 & -3.770 & 1.927 & 2.263 & -1.012 & -1968.103 & -4347.822 & -2205.968 \\
\hline & 0.394 & 2.042 & 2.025 & 0.721 & 5.964 & 0.365 & 0.030 & 0.381 & 0.029 \\
\hline \multirow[t]{2}{*}{$\mathrm{E}$} & -0.072 & -1.032 & 0.493 & -0.005 & -0.618 & -0.382 & -0.117 & -0.706 & -0.147 \\
\hline & 0.044 & 1.727 & 0.297 & 0.007 & 2.697 & 0.518 & 0.000 & 1.856 & 0.000 \\
\hline \multirow[t]{2}{*}{$\mathrm{S}$} & -0.790 & 2.456 & 0.406 & -2.947 & -2.284 & 0.020 & 1930.307 & 4265.835 & 2163.422 \\
\hline & 0.205 & 1.491 & 0.169 & 1.769 & 3.491 & 0.012 & 0.023 & 0.381 & 0.030 \\
\hline \multirow[t]{2}{*}{$\mathrm{Y}$} & 9.723 & 0.611 & -2.072 & -3.178 & 1.005 & 5.211 & 8521.893 & 0.970 & -8359.564 \\
\hline & 0.831 & 1.606 & 0.448 & 1.086 & 7.338 & 1.758 & 0.030 & 8.130 & 0.030 \\
\hline
\end{tabular}

Estimates Based on Three-Region Data, 1980-1992.

\begin{tabular}{|c|c|c|c|c|c|c|c|c|c|}
\hline \multirow[b]{2}{*}{ Equation } & \multicolumn{3}{|c|}{ REN0 } & \multicolumn{3}{|c|}{$\overline{\text { REN1 }}$} & \multicolumn{3}{|c|}{ REN2 } \\
\hline & $\mathrm{H}$ & $\mathrm{E}$ & $\mathrm{S}$ & $\mathrm{H}$ & $\mathrm{E}$ & $\mathrm{S}$ & $\mathrm{H}$ & $\mathrm{E}$ & $\mathrm{S}$ \\
\hline \multirow[t]{2}{*}{$\mathrm{H}$} & 1.361 & -3.851 & 0.653 & 269.525 & 67.507 & -300.432 & 0.306 & -0.895 & -1.793 \\
\hline & 0.187 & 0.564 & 0.050 & 0.255 & 0.328 & 0.238 & 0.242 & 2.120 & 1.538 \\
\hline \multirow[t]{2}{*}{$\mathrm{E}$} & -1.016 & -1.149 & 0.909 & -1.412 & -0.005 & 0.352 & -0.109 & -0.977 & 0.116 \\
\hline & 0.852 & 1.652 & 0.634 & 0.036 & 0.012 & 0.009 & 0.152 & 3.794 & 0.168 \\
\hline \multirow[t]{2}{*}{$\mathrm{S}$} & 0.196 & -10.966 & 10.437 & -259.366 & -34.946 & 257.017 & -0.848 & 0.452 & 0.450 \\
\hline & 0.072 & 3.368 & 1.821 & 0.274 & 0.175 & 0.224 & 1.339 & 0.891 & 0.474 \\
\hline \multirow[t]{2}{*}{$\mathrm{Y}$} & 1.838 & 1.256 & 0.334 & -36.600 & 1.065 & 37.294 & 2.382 & 0.970 & -0.054 \\
\hline & 0.573 & 8.870 & 0.247 & 0.237 & 23.589 & 0.249 & 0.787 & 8.443 & 0.022 \\
\hline
\end{tabular}




\section{Notes to Tables 11-31}

1. Own- and cross-price elasticities for a given equation read across the page in each table. Income elasticities for each equation are the last line reported, under "Y".

2. The elasticities themselves appear opposite the expenditure category identifier. Below each estimated elasticity is its $Z$-ratio, being the absolute value of the ratio of the estimated elasticity to its standard error.

3. Standard errors for the elasticities are calculated using an approximation based on a quadratic form of the derivatives of the elasticity functions with respect to the parameters (evaluated at the estimated parameters), with the estimated variance-covariance matrix of the parameters.

4. Where an item in the tables is reported with a value of " 0.000 ", the value is zero to three decimal places. 
Table A1: ACCRA Data, 1989, Fourth Quarter

\begin{tabular}{|c|c|c|c|c|c|c|c|}
\hline Anchorage & 127.6 & 134.1 & 127.3 & 92.5 & 120.9 & 185.0 & 126.6 \\
\hline Annaheim & 130.9 & 101.5 & 222.2 & 73.8 & 106.7 & 133.0 & 111.6 \\
\hline Atlanta & 106.2 & 100.8 & 112.7 & 116.1 & 101.8 & 120.7 & 99.3 \\
\hline $\begin{array}{l}\text { Baltimore } \\
\text { Boston }\end{array}$ & 111.1 & 107.8 & 113.7 & 108.9 & 116.3 & 114.5 & 108.7 \\
\hline Buffalo & 107.9 & 103.6 & 113.5 & 116.9 & 106.0 & 101.6 & 105.2 \\
\hline Chicago & 120.1 & 111.2 & 145.0 & 124.3 & 113.8 & 112.0 & 109.9 \\
\hline $\begin{array}{l}\text { Cincinnatti } \\
\text { Cleveland }\end{array}$ & 102.4 & 97.7 & 108.4 & 110.6 & 106.8 & 91.2 & 98.5 \\
\hline Dallas & 104.2 & 105.2 & 103.7 & 109.7 & 109.7 & 107.0 & 99.0 \\
\hline (Fort Worth) & 97.2 & 102.7 & 81.3 & 109.5 & 105.7 & $\begin{array}{l}107.1 \\
1150\end{array}$ & 95.4 \\
\hline $\begin{array}{l}\text { Denver } \\
\text { Detroit }\end{array}$ & 102.1 & 89.9 & 110.1 & 97.5 & 107.1 & 115.9 & 99.5 \\
\hline $\begin{array}{l}\text { Honolulu } \\
\text { Houston }\end{array}$ & 99.1 & 105.6 & 82.0 & 94.1 & 112.6 & 102.0 & 103.1 \\
\hline Kansas City & 94.8 & 88.3 & 91.9 & 94.7 & 96.4 & 95.2 & 99.8 \\
\hline Los Angeles & 129.2 & 101.5 & 210.2 & 75.9 & 110.2 & 130.6 & 113.0 \\
\hline Miami & 113.5 & 101.2 & 125.9 & 127.7 & 105.1 & 130.5 & 106.0 \\
\hline $\begin{array}{l}\text { Milwaukee } \\
\text { (Kenosha) }\end{array}$ & 104.5 & 103.4 & 118.3 & 102.5 & 107.6 & 86.2 & 98.6 \\
\hline Minneapolis & 101.5 & 92.2 & 107.6 & 99.4 & 110.5 & 102.8 & 98.8 \\
\hline $\begin{array}{l}\text { Nassau Suffolk } \\
\text { New York City }\end{array}$ & 162.0 & 121.0 & 257.4 & 195.5 & 126.8 & 139.1 & 123.5 \\
\hline Philadelphia & 129.2 & 114.4 & 146.1 & 173.1 & 110.2 & 140.2 & 114.9 \\
\hline $\begin{array}{l}\text { Pittsburgh } \\
\text { Portland }\end{array}$ & 106.1 & 100.4 & 108.4 & 123.0 & 101.9 & 107.3 & 103.0 \\
\hline San Diego & 129.2 & 102.8 & 210.9 & 76.8 & 119.4 & 129.2 & 107.7 \\
\hline San Jose & 129.9 & 104.7 & 219.8 & 85.4 & 109.1 & 134.3 & 102.7 \\
\hline St Louis & 97.3 & 92.0 & 96.7 & 110.0 & 99.3 & 96.9 & 95.0 \\
\hline $\begin{array}{l}\text { Seattle } \\
\text { Washington DC }\end{array}$ & 113.2 & 112.8 & 129.2 & 64.6 & 121.5 & 143.0 & 109.0 \\
\hline
\end{tabular}

\section{Notes:}

1. Source: American Chamber of Commerce Research Association Inter-city Cost of Living Indicators. Indices are based on specified types and quantities of specific products and services. See the ACCRA reports for further details of goods included in each category, and cities covered.

2. The columns 1-7 above are indices for: all items; grocery items; housing; utilities; transportation; health care; and miscellaneous goods and services respectively.

3. The cities listed above are all of the cities for which households were extracted from the CES data files. The indicated price indices are the ACCRA price data for the the quarter, 1989(4). As discussed previously, these indices reflect price differences across cities at a point in time.

4. Some cities listed above have no price indices stated. These are cities which were not covered by the ACCRA survey in 1989(4). However, these cities were covered in other years by ACCRA surveys. Price indices for those cities in those other survey years were used to construct appropriate indices for those cities in 1989(4), in conjunction with CPI price data. Details of the method of construction of these indices are available on request.

5. Milwaukee is not covered by any of the ACCRA surveys. However, Kenosha, WI is covered, and these indices are used as proxies for Milwaukee. 
Table A2: Descriptive Statistics for 1980-92

Four-Region Data Sets, All Households

\begin{tabular}{|c|c|c|c|c|c|c|}
\hline \multirow[b]{2}{*}{ Equation } & MOR0 & MOR1 & MOR2 & REN0 & "REN1 & REN2 \\
\hline & \multicolumn{6}{|c|}{ Budget Shares } \\
\hline $\mathrm{F}$ & 0.382 & 0.376 & 0.398 & 0.406 & 0.425 & 0.458 \\
\hline A & 0.040 & 0.032 & 0.030 & 0.048 & 0.035 & 0.035 \\
\hline $\mathrm{C}$ & 0.125 & 0.125 & 0.125 & 0.114 & 0.112 & 0.116 \\
\hline $\mathrm{G}$ & 0.115 & 0.109 & 0.108 & 0.119 & 0.118 & 0.106 \\
\hline $\mathrm{O}$ & 0.161 & 0.154 & 0.142 & 0.161 & 0.128 & 0.117 \\
\hline $\mathrm{P}$ & 0.037 & 0.026 & 0.022 & 0.037 & 0.026 & 0.024 \\
\hline $\mathrm{H}$ & 0.026 & 0.066 & 0.068 & 0.015 & 0.053 & 0.055 \\
\hline $\mathrm{E}$ & 0.084 & 0.087 & 0.079 & 0.072 & 0.080 & 0.067 \\
\hline S & 0.029 & 0.025 & 0.027 & 0.028 & 0.023 & 0.022 \\
\hline \multirow[t]{2}{*}{ Sample Sizes } & 1925 . & 1369. & 1766. & 985. & 728. & 604. \\
\hline & \multicolumn{6}{|c|}{ Other Sample Means } \\
\hline $\mathrm{CG}$ & 3562.67 & 4048.98 & 4125.69 & 2703.30 & 2251.29 & 2518.84 \\
\hline Age of Head & 45.01 & 38.00 & 37.76 & 34.54 & 31.92 & 33.31 \\
\hline \multirow[t]{2}{*}{ Total Expend } & 3172.89 & 3451.08 & 3648.83 & 2440.36 & 2349.13 & 2593.83 \\
\hline & \multicolumn{6}{|c|}{ Count Summary for Dummy Variables } \\
\hline MLFP & 1847. & 1341. & 1734. & 915. & 683. & 571. \\
\hline FLFP & 1536. & 1089. & 1207. & 775. & 507. & 375. \\
\hline VH & 1826 . & 1283. & 1664. & 842. & 616. & 501. \\
\hline TOBC & 703. & 553. & 650 & 439. & 353. & 286. \\
\hline
\end{tabular}

\section{Notes to Table A2:}

1. CG indicates the sample means for the conditioning good, $g$.

2. MLFP and FLFP indicate the numbers of males and females employed outside the home respectively.

3. VH indicates the numbers of households owning vehicles.

4. TOBC indicates the numbers of households consuming tobacco products. 
$1980(1)$

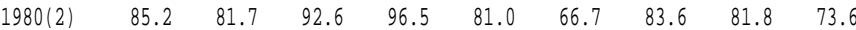

$\begin{array}{llllllllll}1980(3) & 86.3 & 82.2 & 96.7 & 101.0 & 82.8 & 66.9 & 84.2 & 81.7 & 73.5\end{array}$

$\begin{array}{llllllllll}1980(4) & 87.0 & 83.7 & 96.5 & 101.3 & 83.4 & 68.9 & 85.5 & 82.9 & 73.9\end{array}$

$\begin{array}{llllllllll}1980(5) & 87.8 & 83.9 & 95.5 & 101.7 & 84.6 & 69.2 & 86.3 & 83.6 & 73.7\end{array}$

$\begin{array}{llllllllll}1980(6) & 88.7 & 84.7 & 93.4 & 102.2 & 84.9 & 70.3 & 87.4 & 83.0 & 73.9\end{array}$

$\begin{array}{llllllllll}1980(7) & 89.8 & 84.9 & 93.9 & 101.8 & 85.1 & 70.3 & 86.5 & 85.5 & 76.3\end{array}$

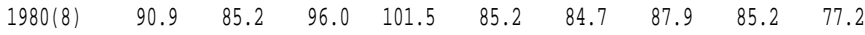

$\begin{array}{llllllllll}1980(9) & 91.4 & 86.7 & 95.2 & 101.0 & 85.6 & 83.1 & 88.9 & 86.9 & 77.5\end{array}$

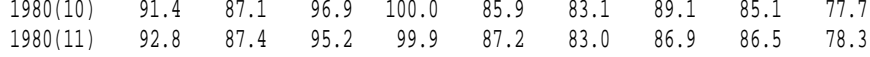

$\begin{array}{llllllllll}1980(12) & 92.8 & 88.1 & 93.1 & 100.7 & 87.7 & 83.4 & 88.8 & 86.8 & 78.4\end{array}$

$\begin{array}{llllllllll}1981(1) & 94.1 & 89.0 & 92.6 & 104.0 & 89.0 & 84.2 & 88.5 & 86.3 & 80.1\end{array}$

$\begin{array}{llllllllll}1981(2) & 93.8 & 89.7 & 92.6 & 111.4 & 90.9 & 87.3 & 89.6 & 86.6 & 81.0\end{array}$

$\begin{array}{llllllllll}1981(3) & 93.8 & 89.9 & 95.4 & 112.9 & 91.2 & 88.6 & 91.6 & 87.5 & 81.2\end{array}$

$\begin{array}{llllllllll}1981(4) & 93.4 & 89.9 & 95.4 & 111.9 & 92.0 & 88.1 & 92.1 & 87.3 & 81.7\end{array}$

$\begin{array}{llllllllll}1981(5) & 93.7 & 91.1 & 95.1 & 110.6 & 92.7 & 88.3 & 90.7 & 88.5 & 81.7\end{array}$

$\begin{array}{llllllllll}1981(6) & 93.8 & 91.6 & 95.1 & 110.0 & 93.0 & 89.4 & 92.3 & 90.4 & 82.4 \\ 1981(7) & 94.7 & 91.8 & 90.2 & 111.4 & 94.0 & 92.7 & 94.0 & 88.9 & 85.2\end{array}$

$\begin{array}{llllllllll}1981(8) & 95.1 & 92.2 & 97.7 & 110.9 & 93.9 & 92.9 & 92.9 & 89.9 & 86.1\end{array}$

$\begin{array}{llllllllll}1981(9) & 96.0 & 93.3 & 100.4 & 110.7 & 94.5 & 93.4 & 94.2 & 92.1 & 86.6\end{array}$

$\begin{array}{llllllllll}1981(10) & 94.7 & 93.3 & 100.4 & 110.3 & 96.0 & 93.7 & 94.4 & 91.9 & 86.5\end{array}$

$\begin{array}{llllllllll}1981(11) & 94.7 & 93.3 & 98.2 & 110.2 & 96.7 & 94.2 & 94.4 & 89.9 & 86.7\end{array}$

$\begin{array}{llllllllll}1981(12) & 95.6 & 93.4 & 98.0 & 110.0 & 97.5 & 94.2 & 96.2 & 94.9 & 87.3\end{array}$

$\begin{array}{llllllllll}1982(1) & 96.7 & 94.6 & 96.4 & 109.3 & 96.8 & 94.1 & 95.6 & 94.6 & 89.1\end{array}$

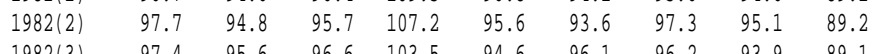

$\begin{array}{rrrrrrrrrr}1982(3) & 97.4 & 95.6 & 96.6 & 103.5 & 94.6 & 96.1 & 96.2 & 93.9 & 89.1 \\ 1982(4) & 98.7 & 96.0 & 98.6 & 98.5 & 93.8 & 96.0 & 96.9 & 95.6 & 89.4\end{array}$

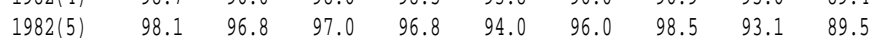

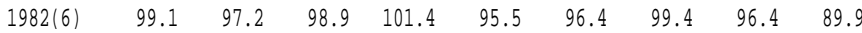

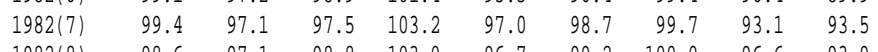

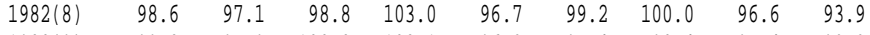

$\begin{array}{llllllllll}1982(9) & 99.2 & 97.9 & 102.8 & 102.1 & 96.3 & 97.4 & 98.4 & 95.4 & 93.3\end{array}$

$\begin{array}{llllllllll}1982(10) & 97.8 & 97.9 & 102.8 & 101.9 & 97.0 & 97.6 & 98.9 & 98.1 & 93.6\end{array}$

$\begin{array}{llllllllll}1982(11) & 97.3 & 98.1 & 101.2 & 101.1 & 96.9 & 99.4 & 99.9 & 97.7 & 95.0\end{array}$

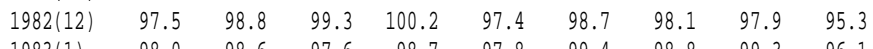

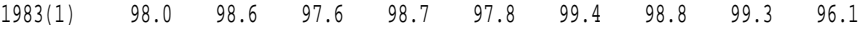

$\begin{array}{llllllllll}1983(2) & 98.8 & 99.1 & 98.2 & 95.2 & 96.6 & 99.7 & 98.7 & 96.9 & 97.7\end{array}$

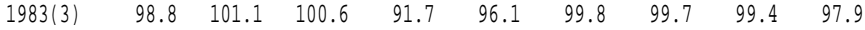

$\begin{array}{llllllllll}1983(4) & 99.3 & 100.8 & 100.4 & 95.8 & 97.3 & 100.0 & 101.4 & 98.1 & 98.3\end{array}$

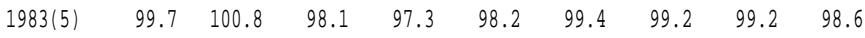

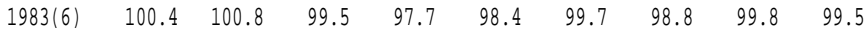

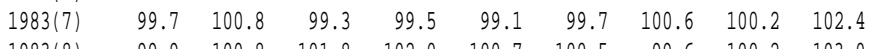

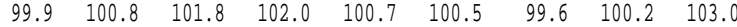

$\begin{array}{llllllllll}1983(9) & 99.8 & 100.7 & 102.7 & 102.9 & 101.4 & 100.1 & 101.8 & 102.1 & 103.1\end{array}$

$\begin{array}{llllllllll}1983(10) & 99.6 & 100.6 & 103.7 & 102.8 & 102.3 & 98.1 & 99.7 & 101.1 & 103.7\end{array}$

$\begin{array}{llllllllll}1983(11) & 99.1 & 100.6 & 102.4 & 102.5 & 102.5 & 100.6 & 101.3 & 101.8 & 103.5\end{array}$

$\begin{array}{lrrrrrrrrr}1983(12) & 99.7 & 100.6 & 99.5 & 101.5 & 102.2 & 100.3 & 100.2 & 103.0 & 103.6\end{array}$

$\begin{array}{llllllllll}1984(1) & 102.0 & 101.4 & 100.0 & 100.1 & 102.9 & 100.8 & 100.3 & 103.9 & 103.9\end{array}$

$\begin{array}{llllllllll}1984(2) & 103.0 & 101.2 & 99.2 & 99.4 & 102.9 & 100.0 & 101.1 & 104.3 & 104.8\end{array}$

$\begin{array}{llllllllll}1984(3) & 102.6 & 102.1 & 99.2 & 98.8 & 103.2 & 100.0 & 102.6 & 100.9 & 104.7\end{array}$

$\begin{array}{llllllllll}1984(4) & 102.0 & 101.9 & 100.5 & 99.0 & 104.1 & 99.9 & 99.9 & 104.5 & 105.6\end{array}$

$\begin{array}{llllllllll}1984(5) & 101.9 & 102.2 & 99.0 & 99.2 & 104.4 & 99.4 & 101.5 & 103.0 & 105.6\end{array}$

$\begin{array}{llllllllll}1984(6) & 101.9 & 102.9 & 97.6 & 99.2 & 104.7 & 100.5 & 103.0 & 103.0 & 105.9\end{array}$

$\begin{array}{llllllllll}1984(7) & 103.2 & 103.8 & 99.0 & 98.5 & 104.8 & 106.6 & 101.2 & 105.3 & 107.5\end{array}$

$\begin{array}{llllllllll}1984(8) & 103.5 & 103.9 & 102.4 & 97.9 & 105.4 & 106.9 & 100.9 & 105.2 & 108.4\end{array}$

$\begin{array}{llllllllll}1984(9) & 102.5 & 103.9 & 103.8 & 98.3 & 105.7 & 106.0 & 104.4 & 105.2 & 109.0\end{array}$

$\begin{array}{llllllllll}1984(10) & 103.1 & 103.9 & 104.3 & 98.5 & 105.2 & 106.3 & 103.3 & 105.3 & 109.3\end{array}$

$\begin{array}{llllllllll}1984(11) & 102.1 & 103.2 & 103.1 & 97.8 & 106.0 & 106.3 & 102.8 & 105.5 & 113.4\end{array}$

$\begin{array}{llllllllll}1984(12) & 102.0 & 102.3 & 102.4 & 97.6 & 106.2 & 106.7 & 100.3 & 105.4 & 114.0\end{array}$

$\begin{array}{llllllllll}1985(1) & 103.8 & 104.3 & 99.6 & 96.7 & 106.3 & 106.5 & 100.6 & 105.9 & 115.8\end{array}$

$\begin{array}{llllllllll}1985(2) & 105.2 & 104.3 & 100.3 & 96.3 & 107.1 & 106.7 & 102.3 & 109.0 & 116.1\end{array}$

$\begin{array}{llllllllll}1985(3) & 106.0 & 104.4 & 101.1 & 97.7 & 107.8 & 107.1 & 101.9 & 109.7 & 117.1\end{array}$

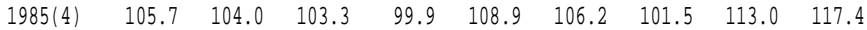

$\begin{array}{llllllllll}1985(5) & 105.7 & 104.4 & 102.4 & 102.4 & 109.4 & 106.4 & 101.0 & 111.6 & 117.7\end{array}$

$\begin{array}{llllllllll}1985(6) & 105.3 & 104.4 & 100.2 & 103.7 & 109.9 & 107.1 & 101.3 & 111.0 & 118.5\end{array}$

$\begin{array}{llllllllll}1985(7) & 106.6 & 105.4 & 100.0 & 104.6 & 109.8 & 110.5 & 100.4 & 111.9 & 119.3\end{array}$

$\begin{array}{llllllllll}1985(8) & 105.6 & 105.4 & 102.7 & 104.5 & 109.5 & 115.5 & 101.1 & 111.9 & 119.8\end{array}$

$\begin{array}{llllllllll}1985(9) & 104.9 & 105.8 & 101.9 & 104.3 & 109.8 & 116.4 & 100.5 & 112.8 & 121.0\end{array}$

$\begin{array}{llllllllll}1985(10) & 105.2 & 108.6 & 101.8 & 104.6 & 111.0 & 116.0 & 101.1 & 112.4 & 121.7\end{array}$

$\begin{array}{llllllllll}1985(11) & 104.5 & 108.5 & 103.3 & 105.1 & 111.1 & 116.0 & 101.3 & 112.7 & 122.6\end{array}$

$\begin{array}{llllllllll}1985(12) & 105.6 & 108.7 & 100.6 & 105.2 & 111.1 & 117.3 & 101.8 & 111.5 & 122.2\end{array}$

$\begin{array}{llllllllll}1986(1) & 105.7 & 108.9 & 100.2 & 104.7 & 111.7 & 116.4 & 100.7 & 114.0 & 122.5\end{array}$

$\begin{array}{llllllllll}1986(2) & 105.7 & 108.8 & 100.0 & 100.0 & 110.4 & 117.1 & 101.4 & 119.2 & 123.5\end{array}$

$\begin{array}{llllllllll}1986(3) & 107.1 & 109.1 & 100.4 & 87.4 & 107.6 & 117.5 & 102.0 & 121.0 & 124.3\end{array}$

$\begin{array}{llllllllll}1986(4) & 106.9 & 110.2 & 99.7 & 73.0 & 103.6 & 116.7 & 102.5 & 121.1 & 125.5\end{array}$

$\begin{array}{llllllllll}1986(5) & 106.9 & 109.8 & 100.0 & 76.9 & 104.7 & 117.6 & 102.5 & 121.2 & 125.0\end{array}$

$\begin{array}{llllllllll}1986(6) & 106.9 & 109.8 & 97.1 & 81.0 & 106.0 & 118.1 & 103.6 & 121.3 & 125.5\end{array}$

$\begin{array}{llllllllll}1986(7) & 107.9 & 110.1 & 100.6 & 76.2 & 105.2 & 125.8 & 104.3 & 121.3 & 127.6\end{array}$ $\begin{array}{llllllllll}1986(9) & 109.6 & 110.0 & 104.8 & 73.1 & 103.2 & 126.0 & 104.5 & 120.4 & 129.0\end{array}$

$\begin{array}{llllllllll}1986(10) & 109.8 & 110.6 & 104.4 & 71.9 & 104.3 & 126.4 & 104.6 & 120.4 & 129.2\end{array}$

$\begin{array}{llllllllll}1986(11) & 108.8 & 110.8 & 103.5 & 70.8 & 104.3 & 127.0 & 104.0 & 120.7 & 129.2\end{array}$

$\begin{array}{llllllllll}1986(12) & 109.2 & 111.6 & 102.8 & 71.0 & 105.1 & 128.7 & 104.8 & 121.3 & 130.0\end{array}$

$\begin{array}{llllllllll}1987(1) & 111.2 & 111.6 & 104.7 & 74.8 & 106.7 & 128.7 & 105.3 & 127.5 & 129.9\end{array}$

$\begin{array}{llllllllll}1987(2) & 111.7 & 111.7 & 103.5 & 77.7 & 106.5 & 129.3 & 106.4 & 127.5 & 130.3\end{array}$

$\begin{array}{llllllllll}1987(3) & 110.7 & 111.5 & 104.3 & 78.7 & 107.0 & 130.1 & 106.9 & 127.7 & 130.7\end{array}$

$\begin{array}{llllllllll}1987(4) & 112.4 & 111.7 & 110.6 & 80.1 & 108.8 & 127.9 & 106.9 & 127.2 & 130.6\end{array}$

$\begin{array}{llllllllll}1987(5) & 113.2 & 111.4 & 106.6 & 81.2 & 109.2 & 126.7 & 108.4 & 128.0 & 131.7\end{array}$

$\begin{array}{llllllllll}1987(6) & 113.4 & 111.6 & 103.7 & 82.8 & 109.9 & 128.9 & 107.1 & 128.3 & 132.9\end{array}$

$\begin{array}{llllllllll}1987(7) & 113.9 & 111.7 & 102.0 & 84.8 & 110.5 & 131.4 & 106.7 & 128.6 & 134.0\end{array}$

$\begin{array}{llllllllll}1987(8) & 114.7 & 111.5 & 110.3 & 86.7 & 110.3 & 130.9 & 106.0 & 128.7 & 134.0\end{array}$

$\begin{array}{llllllllll}1987(9) & 113.7 & 116.3 & 111.3 & 86.3 & 110.7 & 132.9 & 107.1 & 129.3 & 135.3\end{array}$

$\begin{array}{llllllllll}1987(10) & 114.9 & 116.5 & 112.4 & 85.3 & 111.8 & 131.5 & 106.3 & 130.0 & 135.3\end{array}$

$\begin{array}{llllllllll}1987(11) & 114.3 & 116.5 & 113.0 & 85.5 & 112.6 & 131.8 & 106.9 & 129.6 & 135.2\end{array}$

$\begin{array}{llllllllll}1987(12) & 114.2 & 116.5 & 110.3 & 84.9 & 112.9 & 131.7 & 106.4 & 130.6 & 136.6\end{array}$

$\begin{array}{llllllllll}1988(1) & 115.9 & 116.0 & 109.1 & 83.6 & 113.0 & 130.4 & 108.3 & 131.3 & 137.7\end{array}$

$\begin{array}{lllllllllll}1988(2) & 115.0 & 116.0 & 106.9 & 82.4 & 112.8 & 128.8 & 107.1 & 133.1 & 138.7\end{array}$

$\begin{array}{llllllllll}1988(3) & 112.8 & 117.6 & 112.7 & 80.9 & 113.0 & 129.6 & 108.2 & 132.2 & 138.4\end{array}$

$\begin{array}{llllllllll}1988(4) & 115.5 & 117.8 & 107.9 & 81.0 & 112.6 & 131.0 & 107.7 & 132.4 & 139.8\end{array}$

$\begin{array}{llllllllll}1988(5) & 116.9 & 117.8 & 109.2 & 81.8 & 113.6 & 130.3 & 108.6 & 132.1 & 140.7\end{array}$

$\begin{array}{llllllllll}1988(6) & 118.1 & 118.2 & 104.6 & 82.0 & 113.3 & 130.5 & 108.9 & 132.2 & 140.7\end{array}$

$\begin{array}{llllllllll}1988(7) & 120.2 & 118.2 & 108.7 & 83.1 & 113.9 & 129.9 & 108.0 & 131.2 & 143.8\end{array}$

$\begin{array}{llllllllll}1988(8) & 119.8 & 118.2 & 108.9 & 83.6 & 113.6 & 130.1 & 109.0 & 131.0 & 143.5\end{array}$

$\begin{array}{llllllllll}1988(9) & 120.1 & 118.3 & 112.7 & 83.6 & 114.5 & 131.4 & 109.0 & 130.8 & 144.7\end{array}$

$\begin{array}{llllllllll}1988(10) & 121.8 & 118.4 & 113.7 & 83.4 & 115.9 & 133.5 & 108.1 & 131.8 & 145.5\end{array}$

$\begin{array}{llllllllll}1988(11) & 119.7 & 118.3 & 113.8 & 83.8 & 116.2 & 133.3 & 106.8 & 131.9 & 146.1\end{array}$

$\begin{array}{llllllllll}1988(12) & 120.4 & 122.2 & 110.3 & 84.1 & 116.3 & 134.2 & 107.5 & 132.6 & 146.2\end{array}$

$\begin{array}{llllllllll}1989(1) & 122.4 & 122.3 & 111.3 & 84.4 & 116.8 & 137.2 & 110.4 & 139.4 & 146.9\end{array}$

$\begin{array}{llllllllll}1989(2) & 123.0 & 123.8 & 108.9 & 85.0 & 116.3 & 136.4 & 109.9 & 140.4 & 147.8\end{array}$

$\begin{array}{llllllllll}1989(3) & 125.0 & 124.7 & 101.0 & 85.0 & 116.4 & 136.2 & 110.6 & 139.5 & 148.8\end{array}$

$\begin{array}{llllllllll}1989(4) & 125.7 & 124.9 & 102.7 & 90.7 & 118.6 & 138.1 & 112.0 & 142.3 & 151.1\end{array}$

$\begin{array}{llllllllll}1989(5) & 126.1 & 126.4 & 102.8 & 97.3 & 121.4 & 138.4 & 111.5 & 141.3 & 152.0\end{array}$

$\begin{array}{llllllllll}1989(6) & 128.3 & 130.3 & 98.4 & 98.2 & 121.8 & 138.6 & 111.8 & 140.3 & 155.1\end{array}$

$\begin{array}{llllllllll}1989(7) & 129.3 & 130.6 & 96.4 & 98.2 & 121.4 & 140.7 & 111.7 & 141.1 & 156.0\end{array}$

$\begin{array}{llllllllll}1989(8) & 128.2 & 132.6 & 91.8 & 94.0 & 119.9 & 150.0 & 111.2 & 143.4 & 155.9\end{array}$

$\begin{array}{llllllllll}1989(9) & 129.1 & 132.3 & 93.4 & 93.8 & 120.1 & 149.2 & 109.8 & 143.5 & 159.9\end{array}$

$\begin{array}{llllllllll}1989(10) & 130.6 & 132.9 & 97.8 & 94.0 & 121.2 & 148.5 & 110.6 & 141.6 & 164.1\end{array}$

$\begin{array}{llllllllll}1989(11) & 130.7 & 135.2 & 95.8 & 93.0 & 121.5 & 139.2 & 110.2 & 147.0 & 166.1\end{array}$

$\begin{array}{llllllllll}1989(12) & 131.9 & 133.3 & 92.0 & 92.7 & 121.6 & 139.2 & 110.6 & 149.2 & 159.8\end{array}$

$\begin{array}{llllllllll}1990(1) & 132.9 & 134.0 & 87.8 & 95.6 & 123.4 & 140.2 & 109.5 & 152.5 & 160.4\end{array}$

$\begin{array}{llllllllll}1990(2) & 135.5 & 134.2 & 94.4 & 96.1 & 123.3 & 151.0 & 113.1 & 153.0 & 160.1\end{array}$ $\begin{array}{llllllllll}1990(3) & 135.9 & 136.0 & 101.4 & 95.3 & 122.9 & 151.8 & 113.5 & 152.3 & 162.1\end{array}$

$\begin{array}{lllllllllll}1990(4) & 134.5 & 136.0 & 107.1 & 95.1 & 122.6 & 154.6 & 116.3 & 153.6 & 164.2\end{array}$

$\begin{array}{llllllllll}1990(5) & 134.6 & 136.1 & 108.7 & 95.8 & 122.8 & 154.6 & 116.3 & 152.7 & 165.2\end{array}$

$\begin{array}{llllllllll}1990(6) & 135.0 & 136.1 & 102.8 & 98.2 & 123.1 & 158.0 & 114.7 & 155.4 & 166.2\end{array}$

$\begin{array}{llllllllll}1990(7) & 135.9 & 139.0 & 100.4 & 98.7 & 122.4 & 158.4 & 113.4 & 158.1 & 169.2\end{array}$

$\begin{array}{llllllllll}1990(8) & 136.8 & 139.1 & 100.7 & 105.1 & 123.1 & 158.1 & 113.2 & 151.5 & 170.4\end{array}$

$\begin{array}{llllllllll}1990(9) & 135.7 & 138.4 & 105.9 & 116.4 & 125.5 & 161.2 & 114.8 & 154.7 & 171.0\end{array}$

$\begin{array}{llllllllll}1990(10) & 137.2 & 139.2 & 105.1 & 127.0 & 129.6 & 161.2 & 114.8 & 153.3 & 171.5\end{array}$

$\begin{array}{llllllllll}1990(11) & 137.1 & 138.6 & 101.9 & 128.9 & 131.0 & 165.3 & 115.4 & 153.8 & 174.3\end{array}$

$\begin{array}{llllllllll}1990(12) & 138.8 & 140.0 & 100.6 & 129.0 & 131.6 & 168.9 & 114.1 & 156.2 & 174.6\end{array}$

$\begin{array}{llllllllll}1991(1) & 139.0 & 152.0 & 103.0 & 116.9 & 129.6 & 168.8 & 112.6 & 164.5 & 176.0\end{array}$

$\begin{array}{llllllllll}1991(2) & 139.2 & 155.5 & 103.1 & 105.8 & 128.2 & 168.6 & 116.2 & 173.7 & 178.4\end{array}$

$\begin{array}{llllllllll}1991(3) & 139.7 & 154.9 & 106.6 & 101.9 & 127.4 & 162.2 & 115.6 & 156.3 & 178.8\end{array}$

$\begin{array}{llllllllll}1991(4) & 140.2 & 154.5 & 105.5 & 103.3 & 127.6 & 157.6 & 115.5 & 157.6 & 180.5\end{array}$

$\begin{array}{llllllllll}1991(5) & 140.1 & 154.9 & 106.0 & 105.4 & 128.0 & 159.2 & 118.4 & 157.5 & 181.3\end{array}$

$\begin{array}{llllllllll}1991(6) & 140.4 & 155.3 & 98.9 & 105.2 & 128.5 & 159.2 & 117.6 & 157.4 & 182.0\end{array}$

$\begin{array}{llllllllll}1991(7) & 139.4 & 155.4 & 92.8 & 103.8 & 129.8 & 159.3 & 118.6 & 161.8 & 184.7\end{array}$

$\begin{array}{llllllllll}1991(8) & 138.3 & 156.2 & 100.3 & 103.0 & 129.7 & 161.0 & 118.2 & 160.2 & 186.5\end{array}$

$\begin{array}{llllllllll}1991(9) & 137.8 & 156.7 & 107.6 & 106.3 & 130.3 & 157.2 & 116.5 & 161.4 & 186.7\end{array}$

$\begin{array}{llllllllll}1991(10) & 137.3 & 157.6 & 104.1 & 105.8 & 131.9 & 157.2 & 115.7 & 160.9 & 188.4\end{array}$

$\begin{array}{llllllllll}1991(11) & 137.3 & 157.7 & 108.4 & 106.0 & 132.9 & 161.7 & 116.9 & 159.8 & 188.5\end{array}$

$\begin{array}{lllllllllll}1991(12) & 137.2 & 158.7 & 108.6 & 106.2 & 133.2 & 164.1 & 118.1 & 160.8 & 189.6\end{array}$

$\begin{array}{llllllllll}1992(1) & 138.7 & 159.1 & 104.9 & 103.5 & 132.6 & 163.5 & 118.4 & 162.1 & 188.0\end{array}$

$\begin{array}{llllllllll}1992(2) & 139.5 & 159.3 & 100.5 & 100.2 & 132.0 & 164.0 & 117.2 & 167.3 & 188.7\end{array}$

$\begin{array}{llllllllll}1992(3) & 140.6 & 159.9 & 107.1 & 98.3 & 131.4 & 169.2 & 117.8 & 168.4 & 192.1\end{array}$

$\begin{array}{llllllllll}1992(4) & 141.3 & 159.5 & 110.4 & 96.5 & 131.5 & 174.0 & 115.1 & 168.7 & 193.0\end{array}$

$\begin{array}{llllllllll}1992(5) & 141.7 & 159.8 & 110.9 & 99.4 & 132.7 & 160.6 & 115.2 & 169.3 & 195.6\end{array}$

$\begin{array}{llllllllll}1992(6) & 140.8 & 160.2 & 105.8 & 104.4 & 133.7 & 161.2 & 116.9 & 168.6 & 196.4\end{array}$

$\begin{array}{llllllllll}1992(7) & 142.2 & 159.6 & 103.6 & 106.3 & 134.0 & 165.8 & 113.9 & 171.7 & 199.4\end{array}$

$\begin{array}{llllllllll}1992(8) & 141.7 & 159.3 & 107.4 & 106.5 & 133.9 & 157.4 & 113.3 & 168.9 & 199.5\end{array}$

$\begin{array}{llllllllll}1992(9) & 142.0 & 159.9 & 107.0 & 106.1 & 133.6 & 163.6 & 112.6 & 171.8 & 200.4\end{array}$

$\begin{array}{lllllllllll}1992(10) & 141.7 & 159.8 & 107.0 & 107.0 & 134.8 & 160.9 & 112.4 & 171.5 & 202.0\end{array}$

$\begin{array}{llllllllll}1992(11) & 140.8 & 159.3 & 108.8 & 109.8 & 136.0 & 164.1 & 112.2 & 170.3 & 202.0\end{array}$

$\begin{array}{llllllllll}1992(12) & 142.2 & 159.7 & 104.0 & 109.4 & 136.3 & 174.2 & 113.4 & 171.9 & 202.2\end{array}$ 
$1986(8)$ $1986(9)$

$\begin{array}{lllllllll}95.9 & 93.5 & 125.7 & 86.6 & 93.6 & 99.8 & 109.0 & 94.1 & 108.9\end{array}$

$\begin{array}{lllllllllll}\text { Year(month) } & \text { F } & \text { A } & \text { C } & \text { G } & \text { O } & \text { P } & \text { H } & \text { E } & \text { S }\end{array}$

$\begin{array}{llllllllll}1980(1) & 74.9 & 69.1 & 105.8 & 105.0 & 71.6 & 51.8 & 84.9 & 63.9 & 60.5\end{array}$

$\begin{array}{llllllllll}1980(2) & 74.6 & 69.4 & 111.1 & 114.3 & 73.5 & 52.8 & 87.2 & 63.9 & 62.1\end{array}$

$\begin{array}{llllllllll}1980(3) & 75.5 & 69.9 & 116.0 & 119.7 & 75.1 & 53.0 & 87.8 & 63.9 & 62.0\end{array}$

$\begin{array}{llllllllll}1980(4) & 76.1 & 71.1 & 115.7 & 120.0 & 75.6 & 54.5 & 89.1 & 64.8 & 62.4\end{array}$

$\begin{array}{llllllllll}1980(5) & 76.9 & 71.3 & 114.5 & 120.5 & 76.7 & 54.8 & 90.0 & 65.3 & 62.2\end{array}$

$\begin{array}{llllllllll}1980(6) & 77.6 & 72.0 & 112.0 & 121.1 & 77.0 & 55.7 & 91.1 & 64.9 & 62.4\end{array}$

$\begin{array}{llllllllll}1980(7) & 78.6 & 72.2 & 112.6 & 120.6 & 77.2 & 55.7 & 90.2 & 66.8 & 64.4\end{array}$

$\begin{array}{llllllllll}1980(8) & 79.6 & 72.4 & 115.1 & 120.3 & 77.3 & 67.1 & 91.6 & 66.6 & 65.2\end{array}$

$\begin{array}{llllllllll}1980(9) & 80.0 & 73.7 & 114.2 & 119.7 & 77.6 & 65.8 & 92.7 & 67.9 & 65.4\end{array}$

$\begin{array}{llllllllll}1980(10) & 80.0 & 74.0 & 116.2 & 118.5 & 77.9 & 65.8 & 92.9 & 66.5 & 65.6\end{array}$

$\begin{array}{llllllllll}1980(11) & 81.2 & 74.3 & 114.2 & 118.4 & 79.1 & 65.7 & 90.6 & 67.6 & 66.1\end{array}$

$\begin{array}{llllllllll}1980(12) & 81.2 & 74.9 & 111.7 & 119.3 & 79.5 & 66.0 & 92.6 & 67.8 & 66.2\end{array}$

$\begin{array}{llllllllll}1981(1) & 82.4 & 75.6 & 111.1 & 123.2 & 80.7 & 66.7 & 92.3 & 67.5 & 67.6\end{array}$

$\begin{array}{llllllllll}1981(2) & 82.1 & 76.2 & 111.1 & 132.0 & 82.4 & 69.1 & 93.4 & 67.7 & 68.4\end{array}$

$\begin{array}{llllllllll}1981(3) & 82.1 & 76.4 & 114.4 & 133.8 & 82.7 & 70.1 & 95.5 & 68.4 & 68.5\end{array}$

$\begin{array}{llllllllll}1981(4) & 81.8 & 76.4 & 114.4 & 132.6 & 83.4 & 69.7 & 96.0 & 68.2 & 69.0\end{array}$

$\begin{array}{llllllllll}1981(5) & 82.0 & 77.4 & 114.1 & 131.1 & 84.1 & 69.9 & 94.6 & 69.2 & 69.0\end{array}$

$\begin{array}{llllllllll}1981(6) & 82.1 & 77.8 & 114.1 & 130.3 & 84.4 & 70.8 & 96.2 & 70.7 & 69.6\end{array}$

$\begin{array}{llllllllll}1981(7) & 82.9 & 78.0 & 108.2 & 132.0 & 85.3 & 73.4 & 98.0 & 69.5 & 71.9\end{array}$

$\begin{array}{llllllllll}1981(8) & 83.2 & 78.4 & 117.2 & 131.4 & 85.2 & 73.5 & 96.9 & 70.3 & 72.7 \\ 1981(9) & 84.0 & 79.3 & 120.4 & 131.2 & 85.7 & 73.9 & 98.2 & 72.0 & 73.1\end{array}$

$\begin{array}{llllllllll}1981(10) & 82.9 & 79.3 & 120.4 & 130.7 & 87.1 & 74.2 & 98.4 & 71.8 & 73.0\end{array}$

$\begin{array}{llllllllll}1981(11) & 82.9 & 79.3 & 117.8 & 130.6 & 87.7 & 74.6 & 98.4 & 70.3 & 73.2\end{array}$

$\begin{array}{llllllllll}1981(12) & 83.7 & 79.4 & 117.5 & 130.3 & 88.4 & 74.6 & 100.3 & 74.2 & 73.7\end{array}$

$\begin{array}{llllllllll}1982(1) & 84.6 & 80.4 & 115.6 & 129.5 & 87.8 & 74.5 & 99.7 & 73.9 & 75.2\end{array}$

$\begin{array}{llllllllll}1982(2) & 85.5 & 80.6 & 114.8 & 127.0 & 86.7 & 74.1 & 101.4 & 74.3 & 75.3\end{array}$

$\begin{array}{llllllllll}1982(3) & 85.3 & 81.2 & 115.9 & 122.6 & 85.8 & 76.1 & 100.3 & 73.4 & 75.2\end{array}$

$\begin{array}{llllllllll}1982(4) & 86.4 & 81.6 & 118.3 & 116.7 & 85.1 & 76.0 & 101.0 & 74.7 & 75.5 \\ 1982(5) & 85.9 & 82.3 & 116.3 & 114.7 & 85.3 & 76.0 & 102.7 & 72.8 & 75.5\end{array}$

$\begin{array}{llllllllll}1982(6) & 86.7 & 82.6 & 118.6 & 120.2 & 86.6 & 76.3 & 103.6 & 75.3 & 75.9\end{array}$

$\begin{array}{llllllllll}1982(7) & 87.0 & 82.5 & 116.9 & 122.3 & 88.0 & 78.1 & 104.0 & 72.8 & 78.9\end{array}$

$\begin{array}{llllllllll}1982(8) & 86.3 & 82.5 & 118.5 & 122.0 & 87.7 & 78.5 & 104.3 & 75.5 & 79.3\end{array}$

$\begin{array}{lllllllllll}1982(9) & 86.8 & 83.2 & 123.3 & 121.0 & 87.3 & 77.1 & 102.6 & 74.6 & 78.8\end{array}$

$\begin{array}{llllllllll}1982(10) & 85.6 & 83.2 & 123.3 & 120.7 & 88.0 & 77.3 & 103.1 & 76.7 & 79.0\end{array}$

$\begin{array}{llllllllll}1982(11) & 85.2 & 83.4 & 121.4 & 119.8 & 87.9 & 78.7 & 104.2 & 76.4 & 80.2\end{array}$

$\begin{array}{llllllllll}1982(12) & 85.3 & 84.0 & 119.1 & 118.7 & 88.3 & 78.1 & 102.3 & 76.5 & 80.4\end{array}$

$\begin{array}{lllll}1983(1) & 85.8 & 83.8 & 117.1 & 117.0\end{array}$

$\begin{array}{llllllllll}1986(11) & 95.2 & 94.2 & 124.1 & 83.9 & 94.6 & 100.5 & 108.4 & 94.3 & 109.1\end{array}$

$\begin{array}{llllllllll}1986(12) & 95.6 & 94.8 & 123.3 & 84.1 & 95.3 & 101.9 & 109.3 & 94.8 & 109.7\end{array}$

$\begin{array}{llllllllll}1987(1) & 97.3 & 94.8 & 125.6 & 88.6 & 96.8 & 101.9 & 109.8 & 99.7 & 109.6\end{array}$

$\begin{array}{llllllllll}1987(2) & 97.8 & 94.9 & 124.1 & 92.1 & 96.6 & 102.4 & 110.9 & 99.7 & 110.0\end{array}$

$\begin{array}{llllllllll}1987(3) & 96.9 & 94.8 & 125.1 & 93.3 & 97.0 & 103.0 & 111.5 & 99.8 & 110.3\end{array}$

$\begin{array}{llllllllll}1987(4) & 98.4 & 94.9 & 132.7 & 94.9 & 98.7 & 101.3 & 111.5 & 99.4 & 110.2\end{array}$

$\begin{array}{llllllllll}1987(5) & 99.1 & 94.7 & 127.9 & 96.2 & 99.0 & 100.3 & 113.0 & 100.0 & 111.2\end{array}$

$\begin{array}{llllllllll}1987(6) & 99.3 & 94.8 & 124.4 & 98.1 & 99.7 & 102.0 & 111.7 & 100.3 & 112.2\end{array}$

$\begin{array}{llllllllll}1987(7) & 99.7 & 94.9 & 122.3 & 100.5 & 100.2 & 104.0 & 111.3 & 100.5 & 113.1\end{array}$

$\begin{array}{llllllllll}1987(8) & 100.4 & 94.8 & 132.3 & 102.7 & 100.0 & 103.6 & 110.5 & 100.6 & 113.1\end{array}$

$\begin{array}{llllllllll}1987(9) & 99.5 & 98.8 & 133.5 & 102.3 & 100.4 & 105.2 & 111.7 & 101.1 & 114.2\end{array}$

$\begin{array}{llllllllll}1987(10) & 100.6 & 99.0 & 134.8 & 101.1 & 101.4 & 104.1 & 110.8 & 101.6 & 114.2\end{array}$

$\begin{array}{llllllllll}1987(11) & 100.0 & 99.0 & 135.5 & 101.3 & 102.1 & 104.3 & 111.5 & 101.3 & 114.1\end{array}$

$\begin{array}{llllllllll}1987(12) & 100.0 & 99.0 & 132.3 & 100.6 & 102.4 & 104.3 & 110.9 & 102.1 & 115.3\end{array}$

$\begin{array}{llllllllll}1988(1) & 101.4 & 98.6 & 130.9 & 99.1 & 102.5 & 103.2 & 112.9 & 102.6 & 116.2\end{array}$

$\begin{array}{llllllllll}1988(2) & 100.7 & 98.6 & 128.2 & 97.6 & 102.3 & 102.0 & 111.7 & 104.0 & 117.1\end{array}$

$\begin{array}{llllllllll}1988(3) & 98.7 & 99.9 & 135.2 & 95.9 & 102.5 & 102.6 & 112.8 & 103.3 & 116.8\end{array}$

$\begin{array}{llllllllll}1988(4) & 101.1 & 100.1 & 129.4 & 96.0 & 102.1 & 103.7 & 112.3 & 103.5 & 118.0\end{array}$

$\begin{array}{llllllllll}1988(5) & 102.3 & 100.1 & 131.0 & 96.9 & 103.0 & 103.2 & 113.2 & 103.3 & 118.8\end{array}$

$\begin{array}{llllllllll}1988(6) & 103.4 & 100.5 & 125.5 & 97.2 & 102.8 & 103.3 & 113.5 & 103.3 & 118.8\end{array}$

$\begin{array}{llllllllll}1988(7) & 105.2 & 100.5 & 130.4 & 98.5 & 103.3 & 102.8 & 112.6 & 102.6 & 121.4\end{array}$

$\begin{array}{llllllllll}1988(8) & 104.9 & 100.5 & 130.6 & 99.1 & 103.0 & 103.0 & 113.6 & 102.4 & 121.1\end{array}$

$\begin{array}{llllllllll}1988(9) & 105.1 & 100.5 & 135.2 & 99.1 & 103.9 & 104.0 & 113.6 & 102.2 & 122.1\end{array}$

$\begin{array}{llllllllll}1988(10) & 106.6 & 100.6 & 136.4 & 98.8 & 105.1 & 105.7 & 112.7 & 103.0 & 122.8\end{array}$

$\begin{array}{llllllllll}1988(11) & 104.8 & 100.5 & 136.5 & 99.3 & 105.4 & 105.5 & 111.4 & 103.1 & 123.3\end{array}$

$\begin{array}{llllllllll}1988(12) & 105.4 & 103.9 & 132.3 & 99.7 & 105.5 & 106.2 & 112.1 & 103.6 & 123.4\end{array}$

$\begin{array}{llllllllll}1989(1) & 107.1 & 103.9 & 133.5 & 100.0 & 105.9 & 108.6 & 115.1 & 109.0 & 124.0\end{array}$

$\begin{array}{llllllllll}1989(2) & 107.7 & 105.2 & 130.6 & 100.7 & 105.5 & 108.0 & 114.6 & 109.7 & 124.8\end{array}$

$\begin{array}{llllllllll}1989(3) & 109.4 & 106.0 & 121.1 & 100.7 & 105.6 & 107.8 & 115.3 & 109.0 & 125.6\end{array}$

$\begin{array}{llllllllll}1989(4) & 110.0 & 106.1 & 123.2 & 107.5 & 107.6 & 109.3 & 116.8 & 111.2 & 127.5\end{array}$

$\begin{array}{llllllllll}1989(5) & 110.4 & 107.4 & 123.3 & 115.3 & 110.1 & 109.6 & 116.3 & 110.4 & 128.3\end{array}$

$\begin{array}{lllllllllll}1989(6) & 112.3 & 110.7 & 118.0 & 116.4 & 110.5 & 109.7 & 116.6 & 109.7 & 130.9\end{array}$

$\begin{array}{llllllllll}1989(7) & 113.2 & 111.0 & 115.6 & 116.4 & 110.1 & 111.4 & 116.5 & 110.3 & 131.7\end{array}$

$\begin{array}{llllllllll}1989(8) & 112.2 & 112.7 & 110.1 & 111.4 & 108.7 & 118.8 & 115.9 & 112.1 & 131.6\end{array}$

$\begin{array}{llllllllll}1989(9) & 113.0 & 112.4 & 112.0 & 111.1 & 108.9 & 118.1 & 114.5 & 112.2 & 135.0\end{array}$

$\begin{array}{llllllllll}1989(10) & 114.3 & 112.9 & 117.3 & 111.4 & 109.9 & 117.6 & 115.3 & 110.7 & 138.5\end{array}$

$\begin{array}{llllllllll}1989(11) & 114.4 & 114.9 & 114.9 & 110.2 & 110.2 & 110.2 & 114.9 & 114.9 & 140.2\end{array}$

$\begin{array}{llllllllll}1989(12) & 115.5 & 113.3 & 110.3 & 109.8 & 110.3 & 110.2 & 115.3 & 116.6 & 134.9\end{array}$

$\begin{array}{llllllllll}1990(1) & 116.3 & 113.9 & 105.3 & 113.3 & 111.9 & 111.0 & 114.2 & 119.2 & 135.4\end{array}$ $\begin{array}{llllllllll}1983(2) & 86.5 & 84.2 & 117.8 & 112.8 & 87.6 & 78.9 & 102.9 & 75.7 & 82.5\end{array}$

$\begin{array}{llllllllll}1983(3) & 86.5 & 85.9 & 120.7 & 108.7 & 87.2 & 79.0 & 104.0 & 77.7 & 82.6\end{array}$

$\begin{array}{llllllllll}1983(4) & 86.9 & 85.7 & 120.4 & 113.5 & 88.3 & 79.2 & 105.7 & 76.7 & 83.0\end{array}$

$\begin{array}{llllllllll}1983(5) & 87.3 & 85.7 & 117.7 & 115.3 & 89.1 & 78.7 & 103.4 & 77.5 & 83.2\end{array}$

$\begin{array}{llllllllll}1983(6) & 87.9 & 85.7 & 119.3 & 115.8 & 89.2 & 78.9 & 103.0 & 78.0 & 84.0\end{array}$

$\begin{array}{llllllllll}1983(7) & 87.3 & 85.7 & 119.1 & 117.9 & 89.9 & 78.9 & 104.9 & 78.3 & 86.4\end{array}$

$\begin{array}{llllllllll}1983(8) & 87.4 & 85.7 & 122.1 & 120.9 & 91.3 & 79.6 & 103.8 & 78.3 & 86.9\end{array}$

$\begin{array}{llllllllll}1983(9) & 87.4 & 85.6 & 123.2 & 121.9 & 92.0 & 79.2 & 106.1 & 79.8 & 87.0\end{array}$

$\begin{array}{llllllllll}1983(10) & 87.2 & 85.5 & 124.4 & 121.8 & 92.8 & 77.7 & 104.0 & 79.0 & 87.5\end{array}$

$\begin{array}{llllllllll}1983(11) & 86.7 & 85.5 & 122.8 & 121.5 & 93.0 & 79.6 & 105.6 & 79.6 & 87.4\end{array}$

$\begin{array}{llllllllll}1983(12) & 87.3 & 85.5 & 119.3 & 120.3 & 92.7 & 79.4 & 104.5 & 80.5 & 87.4\end{array}$

$\begin{array}{llllllllll}1984(1) & 89.3 & 86.2 & 119.9 & 118.6 & 93.3 & 79.8 & 104.6 & 81.2 & 87.7\end{array}$

$\begin{array}{llllllllll}1984(2) & 90.2 & 86.0 & 119.0 & 117.8 & 93.3 & 79.2 & 105.4 & 81.5 & 88.5 \\ 1984(3) & 89.8 & 86.8 & 119.0 & 117.1 & 93.6 & 79.2 & 107.0 & 78.9 & 88.4\end{array}$

$\begin{array}{llllllllll}1984(3) & 89.8 & 86.8 & 119.0 & 117.1 & 93.6 & 79.2 & 107.0 & 78.9 & 88.4 \\ 1984(4) & 89.3 & 86.6 & 120.5 & 117.3 & 94.4 & 79.1 & 104.2 & 81.7 & 89.1\end{array}$

$\begin{array}{llllllllll}1984(4) & 89.3 & 86.6 & 120.5 & 117.3 & 94.4 & 79.1 & 104.2 & 81.7 & 89.1 \\ 1984(5) & 89.2 & 86.9 & 118.7 & 117.5 & 94.7 & 78.7 & 105.8 & 80.5 & 89.1\end{array}$

$\begin{array}{llllllllll}1984(6) & 89.2 & 87.4 & 117.1 & 117.5 & 95.0 & 79.6 & 107.4 & 80.5 & 89.4\end{array}$

$\begin{array}{llllllllll}1984(7) & 90.3 & 88.2 & 118.7 & 116.7 & 95.1 & 84.4 & 105.5 & 82.3 & 90.7\end{array}$

$\begin{array}{llllllllll}1984(8) & 90.6 & 88.3 & 122.8 & 116.0 & 95.6 & 84.6 & 105.2 & 82.2 & 91.5\end{array}$

$\begin{array}{llllllllll}1984(9) & 89.7 & 88.3 & 124.5 & 116.5 & 95.9 & 83.9 & 108.9 & 82.2 & 92.0\end{array}$

$\begin{array}{llllllllll}1984(10) & 90.2 & 88.3 & 125.1 & 116.7 & 95.4 & 84.2 & 107.7 & 82.3 & 92.3\end{array}$

$\begin{array}{llllllllll}1984(11) & 89.4 & 87.7 & 123.7 & 115.9 & 96.1 & 84.2 & 107.2 & 82.5 & 95.7\end{array}$

$\begin{array}{llllllllll}1984(12) & 89.3 & 86.9 & 122.8 & 115.7 & 96.3 & 84.5 & 104.6 & 82.4 & 96.2\end{array}$

$\begin{array}{llllllllll}1985(1) & 90.9 & 88.6 & 119.5 & 114.6 & 96.4 & 84.3 & 104.9 & 82.8 & 97.7\end{array}$

$\begin{array}{llllllllll}1985(2) & 92.1 & 88.6 & 120.3 & 114.1 & 97.1 & 84.5 & 106.7 & 85.2 & 98.0\end{array}$

$\begin{array}{llllllllll}1985(3) & 92.8 & 88.7 & 121.3 & 115.8 & 97.8 & 84.8 & 106.2 & 85.7 & 98.8\end{array}$

$\begin{array}{llllllllll}1985(4) & 92.5 & 88.4 & 123.9 & 118.4 & 98.8 & 84.1 & 105.8 & 88.3 & 99.1\end{array}$

$\begin{array}{llllllllll}1985(5) & 92.5 & 88.7 & 122.8 & 121.3 & 99.2 & 84.2 & 105.3 & 87.2 & 99.3\end{array}$

$\begin{array}{llllllllll}1985(6) & 92.2 & 88.7 & 120.2 & 122.9 & 99.7 & 84.8 & 105.6 & 86.8 & 100.0\end{array}$

$\begin{array}{llllllllll}1985(7) & 93.3 & 89.6 & 119.9 & 123.9 & 99.6 & 87.5 & 104.7 & 87.5 & 100.7\end{array}$

$\begin{array}{llllllllll}1985(8) & 92.4 & 89.6 & 123.2 & 123.8 & 99.3 & 91.4 & 105.4 & 87.5 & 101.1\end{array}$

$\begin{array}{llllllllll}1985(9) & 91.8 & 89.9 & 122.2 & 123.6 & 99.6 & 92.2 & 104.8 & 88.2 & 102.1\end{array}$

$\begin{array}{llllllllll}1985(10) & 92.1 & 92.3 & 122.1 & 123.9 & 100.7 & 91.8 & 105.4 & 87.9 & 102.7\end{array}$

$\begin{array}{llllllllll}1985(11) & 91.5 & 92.2 & 123.9 & 124.5 & 100.8 & 91.8 & 105.6 & 88.1 & 103.5\end{array}$

$\begin{array}{llllllllll}1985(12) & 92.4 & 92.4 & 120.7 & 124.7 & 100.8 & 92.9 & 106.1 & 87.2 & 103.1\end{array}$

$\begin{array}{llllllllll}1986(1) & 92.5 & 92.5 & 120.2 & 124.1 & 101.3 & 92.2 & 105.0 & 89.1 & 103.4\end{array}$

$\begin{array}{llllllllll}1986(2) & 92.5 & 92.5 & 119.9 & 118.5 & 100.1 & 92.7 & 105.7 & 93.2 & 104.2\end{array}$

$\begin{array}{llllllllll}1986(3) & 93.7 & 92.7 & 120.4 & 103.6 & 97.6 & 93.0 & 106.4 & 94.6 & 104.9\end{array}$

$\begin{array}{llllllllll}1986(4) & 93.6 & 93.7 & 119.6 & 86.5 & 94.0 & 92.4 & 106.9 & 94.7 & 105.9\end{array}$

$\begin{array}{llllllllll}1986(5) & 93.6 & 93.3 & 119.9 & 91.1 & 95.0 & 93.1 & 106.9 & 94.7 & 105.5\end{array}$

$\begin{array}{llllllllll}1986(6) & 93.6 & 93.3 & 116.5 & 96.0 & 96.1 & 93.5 & 108.0 & 94.8 & 105.9\end{array}$

$\begin{array}{llllllllll}1986(7) & 94.4 & 93.6 & 120.7 & 90.3 & 95.4 & 99.6 & 108.7 & 94.8 & 107.7\end{array}$ $\begin{array}{llllllllll}1990(2) & 118.6 & 114.1 & 113.2 & 113.9 & 111.8 & 119.5 & 117.9 & 119.6 & 135.1\end{array}$ $\begin{array}{llllllllll}1990(3) & 119.0 & 115.6 & 121.6 & 112.9 & 111.5 & 120.2 & 118.3 & 119.0 & 136.8\end{array}$ $\begin{array}{llllllllll}1990(4) & 117.7 & 115.6 & 128.5 & 112.7 & 111.2 & 122.4 & 121.3 & 120.1 & 138.6\end{array}$ $\begin{array}{llllllllll}1990(5) & 117.8 & 115.7 & 130.4 & 113.5 & 111.4 & 122.4 & 121.3 & 119.4 & 139.4\end{array}$ $\begin{array}{llllllllll}1990(6) & 118.2 & 115.7 & 123.3 & 116.4 & 111.7 & 125.1 & 119.6 & 121.5 & 140.3\end{array}$ $\begin{array}{llllllllll}1990(7) & 119.0 & 118.1 & 120.4 & 117.0 & 111.0 & 125.4 & 118.2 & 123.6 & 142.8\end{array}$ $\begin{array}{llllllllll}1990(8) & 119.7 & 118.2 & 120.8 & 124.5 & 111.7 & 125.2 & 118.0 & 118.4 & 143.8\end{array}$ $\begin{array}{llllllllll}1990(9) & 118.8 & 117.6 & 127.0 & 137.9 & 113.8 & 127.6 & 119.7 & 120.9 & 144.3\end{array}$ $\begin{array}{llllllllll}1990(10) & 120.1 & 118.3 & 126.1 & 150.5 & 117.5 & 127.6 & 119.7 & 119.8 & 144.8\end{array}$ $\begin{array}{llllllllll}1990(11) & 120.0 & 117.8 & 122.2 & 152.7 & 118.8 & 130.9 & 120.3 & 120.2 & 147.1\end{array}$ $\begin{array}{llllllllll}1990(12) & 121.5 & 119.0 & 120.7 & 152.9 & 119.4 & 133.7 & 119.0 & 122.1 & 147.4\end{array}$ $\begin{array}{llllllllll}1991(1) & 121.7 & 129.2 & 123.5 & 138.5 & 117.5 & 133.6 & 117.4 & 128.6 & 148.6\end{array}$ $\begin{array}{llllllllll}1991(2) & 121.8 & 132.2 & 123.7 & 125.4 & 116.3 & 133.5 & 121.2 & 135.8 & 150.6\end{array}$ $\begin{array}{llllllllll}1991(3) & 122.3 & 131.6 & 127.9 & 120.7 & 115.6 & 128.4 & 120.5 & 122.2 & 150.9\end{array}$ $\begin{array}{llllllllll}1991(4) & 122.7 & 131.3 & 126.5 & 122.4 & 115.7 & 124.8 & 120.4 & 123.2 & 152.4\end{array}$ $\begin{array}{lllllllllll}1991(5) & 122.6 & 131.6 & 127.1 & 124.9 & 116.1 & 126.0 & 123.4 & 123.1 & 153.0\end{array}$ $\begin{array}{llllllllll}1991(6) & 122.9 & 132.0 & 118.6 & 124.7 & 116.5 & 126.0 & 122.6 & 123.0 & 153.6\end{array}$ $\begin{array}{llllllllll}1991(7) & 122.0 & 132.1 & 111.3 & 123.0 & 117.7 & 126.1 & 123.7 & 126.5 & 155.9\end{array}$ $\begin{array}{llllllllll}1991(8) & 121.1 & 132.7 & 120.3 & 122.0 & 117.6 & 127.5 & 123.2 & 125.2 & 157.4\end{array}$ $\begin{array}{llllllllll}1991(9) & 120.6 & 133.2 & 129.1 & 126.0 & 118.2 & 124.5 & 121.5 & 126.2 & 157.6\end{array}$ $\begin{array}{llllllllll}1991(10) & 120.2 & 133.9 & 124.9 & 125.4 & 119.6 & 124.5 & 120.6 & 125.8 & 159.0\end{array}$ $\begin{array}{llllllllll}1991(11) & 120.2 & 134.0 & 130.0 & 125.6 & 120.5 & 128.0 & 121.9 & 124.9 & 159.1\end{array}$ $\begin{array}{llllllllll}1991(12) & 120.1 & 134.9 & 130.3 & 125.8 & 120.8 & 129.9 & 123.1 & 125.7 & 160.0\end{array}$ $\begin{array}{lllllllllll}1992(1) & 121.4 & 135.2 & 125.8 & 122.6 & 120.3 & 129.4 & 123.4 & 126.7 & 158.7\end{array}$ $\begin{array}{llllllllll}1992(2) & 122.1 & 135.4 & 120.5 & 118.7 & 119.7 & 129.8 & 122.2 & 130.8 & 159.3\end{array}$ $\begin{array}{llllllllll}1992(3) & 123.1 & 135.9 & 128.5 & 116.5 & 119.2 & 133.9 & 122.8 & 131.6 & 162.1\end{array}$ $\begin{array}{llllllllll}1992(4) & 123.7 & 135.6 & 132.4 & 114.3 & 119.3 & 137.8 & 120.0 & 131.9 & 162.9\end{array}$ $\begin{array}{llllllllll}1992(5) & 124.0 & 135.8 & 133.0 & 117.8 & 120.4 & 127.1 & 120.1 & 132.3 & 165.1\end{array}$ $\begin{array}{lllllllllll}1992(6) & 123.2 & 136.1 & 126.9 & 123.7 & 121.3 & 127.6 & 121.9 & 131.8 & 165.8\end{array}$ $\begin{array}{llllllllll}1992(7) & 124.5 & 135.6 & 124.3 & 126.0 & 121.5 & 131.3 & 118.8 & 134.2 & 168.3\end{array}$ $\begin{array}{llllllllll}1992(8) & 124.0 & 135.4 & 128.8 & 126.2 & 121.4 & 124.6 & 118.1 & 132.0 & 168.4\end{array}$ $\begin{array}{llllllllll}1992(9) & 124.3 & 135.9 & 128.3 & 125.7 & 121.2 & 129.5 & 117.4 & 134.3 & 169.2\end{array}$ $\begin{array}{llllllllll}1992(10) & 124.0 & 135.8 & 128.3 & 126.8 & 122.3 & 127.4 & 117.2 & 134.1 & 170.5\end{array}$ $\begin{array}{llllllllll}1992(11) & 123.2 & 135.4 & 130.5 & 130.1 & 123.4 & 129.9 & 117.0 & 133.1 & 170.5\end{array}$ $\begin{array}{llllllllll}1992(12) & 124.5 & 135.7 & 124.7 & 129.6 & 123.6 & 137.9 & 118.2 & 134.4 & 170.7\end{array}$ 


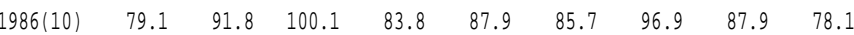
$\begin{array}{llllllllll}1986(11) & 79.9 & 91.6 & 99.1 & 81.9 & 87.9 & 85.7 & 97.0 & 87.9 & 78.3\end{array}$ $\begin{array}{lllllllll}\text { Year (month) } & \text { F } & \text { A } & \text { C } & \text { G } & 0 & \text { P }\end{array}$

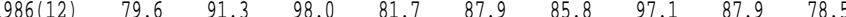

$\begin{array}{llllllllll}1987(1) & 80.2 & 91.9 & 97.8 & 82.9 & 88.2 & 85.3 & 98.1 & 88.3 & 79.0\end{array}$

$\begin{array}{llllllllll}1987(2) & 81.2 & 91.9 & 97.2 & 89.7 & 89.4 & 84.7 & 98.4 & 88.4 & 79.3\end{array}$

$\begin{array}{llllllllll}1980(1) & 60.6 & 67.4 & 79.0 & 102.8 & 69.7 & 46.2 & 76.9 & 60.9 & 45.2\end{array}$

$\begin{array}{llllllllll}1980(2) & 60.9 & 67.8 & 79.7 & 109.7 & 70.1 & 47.2 & 77.4 & 61.3 & 45.4\end{array}$

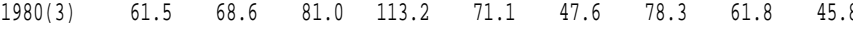

$\begin{array}{llllllllll}1980(4) & 61.7 & 69.6 & 82.2 & 113.9 & 72.0 & 48.2 & 79.1 & 62.2 & 46.3\end{array}$

$\begin{array}{llllllllll}1980(5) & 62.1 & 70.1 & 82.9 & 113.8 & 72.3 & 49.1 & 79.6 & 63.3 & 46.5\end{array}$

$\begin{array}{llllllllll}1980(6) & 62.3 & 70.6 & 83.7 & 114.2 & 72.6 & 49.8 & 79.9 & 64.3 & 46.8 \\ 1980(7) & 62.7 & 71.0 & 84.1 & 115.2 & 73.0 & 50.8 & 80.6 & 64.7 & 46.9\end{array}$

$\begin{array}{llllllllll}1980(8) & 63.8 & 71.4 & 84.4 & 115.6 & 73.4 & 51.9 & 81.3 & 65.1 & 47.2\end{array}$

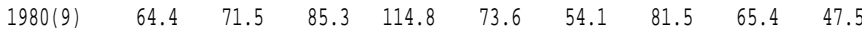

$\begin{array}{llllllllll}1980(10) & 64.9 & 71.8 & 86.0 & 114.0 & 74.1 & 56.3 & 81.7 & 65.7 & 47.8 \\ 1980(11) & 65.4 & 72.0 & 86.8 & 113.5 & 74.6 & 57.1 & 82.1 & 65.9 & 48.2\end{array}$

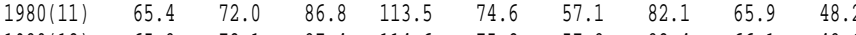

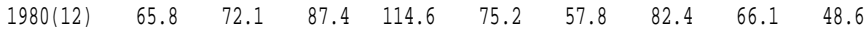

$\begin{array}{llllllllll}1981(1) & 66.7 & 73.6 & 87.0 & 116.7 & 76.5 & 58.5 & 83.4 & 67.0 & 49.1 \\ 1981(2) & 67.2 & 74.9 & 86.6 & 123.7 & 77.8 & 59.2 & 84.4 & 67.8 & 49.7\end{array}$

$\begin{array}{llllllllll}1981(3) & 67.7 & 75.7 & 87.5 & 126.9 & 78.5 & 60.7 & 84.9 & 68.8 & 50.0\end{array}$

$\begin{array}{llllllllll}1981(4) & 68.2 & 76.6 & 88.6 & 127.7 & 79.2 & 62.2 & 85.6 & 69.7 & 50.2\end{array}$

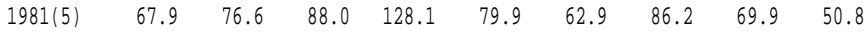

$\begin{array}{llllllllll}1981(6) & 68.3 & 76.6 & 87.2 & 128.1 & 80.6 & 63.5 & 86.8 & 70.1 & 51.5 \\ 1981(7) & 68.8 & 77.2 & 87.8 & 128.2 & 81.4 & 64.4 & 87.2 & 71.1 & 52.3\end{array}$

$\begin{array}{llllllllll}1981(7) & 68.8 & 77.2 & 87.8 & 128.2 & 81.4 & 64.4 & 87.2 & 71.1 & 52.3 \\ 1981(8) & 69.3 & 77.8 & 88.3 & 128.8 & 82.2 & 65.3 & 87.4 & 72.1 & 53.2\end{array}$

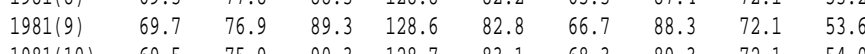

$\begin{array}{llllllllll}1981(10) & 69.5 & 75.9 & 90.3 & 128.7 & 83.1 & 68.3 & 89.3 & 72.1 & 54.0 \\ 1981(11) & 69.1 & 76.2 & 90.1 & 128.3 & 83.5 & 68.2 & 89.7 & 72.4 & 54.7\end{array}$

$\begin{array}{llllllllll}1981(12) & 69.3 & 76.6 & 90.1 & 127.8 & 84.0 & 68.2 & 90.1 & 72.8 & 55.1\end{array}$

$\begin{array}{llllllllll}1982(1) & 70.4 & 76.8 & 90.0 & 126.7 & 83.5 & 67.9 & 90.6 & 73.4 & 55.6\end{array}$

$\begin{array}{llllllllll}1982(2) & 71.1 & 77.1 & 89.9 & 124.0 & 83.1 & 67.6 & 91.0 & 74.1 & 56.1\end{array}$

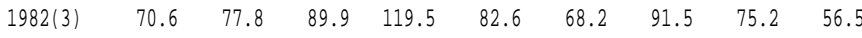

$\begin{array}{llllllllll}1982(4) & 70.5 & 78.6 & 90.1 & 115.8 & 81.9 & 68.7 & 92.0 & 76.2 & 56.9\end{array}$

$\begin{array}{llllllllll}1982(5) & 71.2 & 78.7 & 89.7 & 116.9 & 83.0 & 69.4 & 92.3 & 76.3 & 57.5 \\ 1982(6) & 71.5 & 78.7 & 89.5 & 120.8 & 84.2 & 70.1 & 92.5 & 76.3 & 58.1\end{array}$

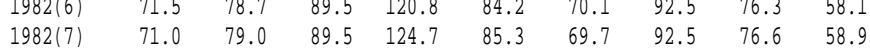

$\begin{array}{llllllllll}1982(8) & 70.7 & 79.3 & 89.5 & 125.1 & 86.4 & 69.2 & 92.5 & 77.0 & 59.8\end{array}$

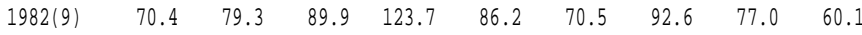

$\begin{array}{llllllllll}1982(10) & 70.6 & 79.2 & 90.5 & 122.0 & 86.1 & 71.9 & 92.8 & 76.9 & 60.4 \\ 1982(11) & 70.4 & 79.2 & 90.2 & 120.7 & 85.6 & 71.9 & 92.9 & 77.6 & 60.9\end{array}$

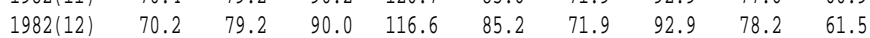

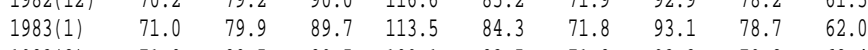

$1983(2)$

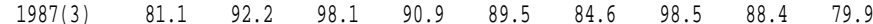

$\begin{array}{llllllllll}1987(4) & 81.3 & 92.6 & 98.3 & 92.2 & 90.3 & 84.3 & 99.7 & 90.8 & 79.9\end{array}$

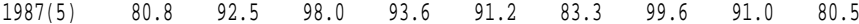

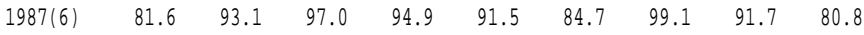

$\begin{array}{llllllllll}1987(7) & 80.8 & 93.0 & 94.4 & 95.7 & 91.9 & 85.3 & 101.5 & 92.7 & 81.7\end{array}$

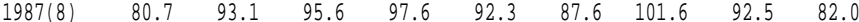

$\begin{array}{llllllllll}1987(9) & 80.9 & 93.3 & 99.6 & 98.9 & 92.4 & 88.1 & 100.5 & 92.6 & 81.6\end{array}$

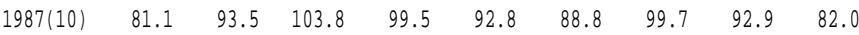

$\begin{array}{llllllllll}1987(11) & 81.2 & 93.6 & 103.2 & 98.9 & 93.4 & 88.7 & 98.5 & 92.4 & 82.3\end{array}$

$\begin{array}{llllllllll}1987(12) & 82.2 & 93.7 & 100.4 & 97.3 & 93.2 & 88.7 & 98.5 & 92.4 & 82.4\end{array}$

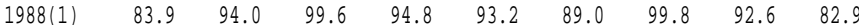

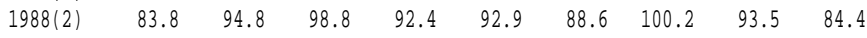

$\begin{array}{llllllllll}1988(3) & 85.0 & 95.6 & 100.7 & 91.3 & 92.5 & 88.7 & 100.1 & 92.3 & 84.7\end{array}$

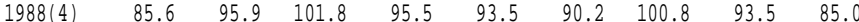

$\begin{array}{llllllllll}1988(5) & 86.1 & 95.8 & 101.5 & 98.2 & 94.4 & 91.0 & 101.3 & 93.5 & 85.4\end{array}$

$\begin{array}{llllllllll}1988(6) & 86.2 & 95.8 & 99.9 & 98.9 & 94.9 & 91.7 & 101.3 & 93.4 & 85.8\end{array}$

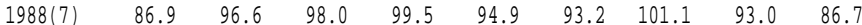

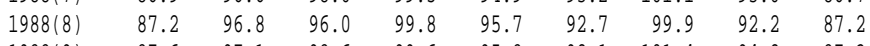

$\begin{array}{llllllllll}1988(9) & 87.6 & 97.1 & 98.6 & 99.6 & 95.8 & 92.1 & 101.4 & 94.2 & 87.3\end{array}$

$\begin{array}{llllllllll}1988(10) & 88.2 & 97.5 & 100.0 & 98.0 & 95.9 & 91.8 & 101.8 & 96.7 & 87.9\end{array}$

$\begin{array}{llllllllll}1988(11) & 87.8 & 97.5 & 100.9 & 95.3 & 95.8 & 91.7 & 101.6 & 97.5 & 88.1\end{array}$

$\begin{array}{llllllllll}1988(12) & 88.5 & 97.1 & 100.2 & 92.1 & 95.4 & 94.1 & 101.5 & 97.9 & 88.5\end{array}$

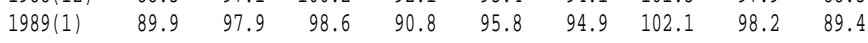

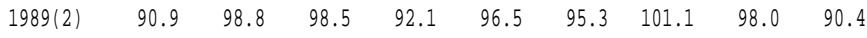

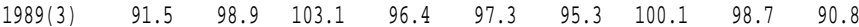

$\begin{array}{llllllllll}1989(4) & 92.1 & 99.4 & 103.8 & 114.1 & 100.9 & 95.4 & 101.1 & 99.5 & 91.4\end{array}$

$\begin{array}{llllllllll}1989(5) & 92.8 & 99.9 & 103.3 & 117.9 & 102.0 & 96.1 & 101.4 & 99.6 & 91.8\end{array}$

$\begin{array}{llllllllll}1989(6) & 92.1 & 99.8 & 101.1 & 116.3 & 101.4 & 97.7 & 101.8 & 99.4 & 92.3\end{array}$

$\begin{array}{lllrrrrrrr}1989(7) & 92.2 & 100.1 & 98.6 & 113.2 & 100.7 & 97.9 & 102.4 & 100.0 & 93.4\end{array}$

$\begin{array}{llllllllll}1989(8) & 92.4 & 100.4 & 98.9 & 109.2 & 99.9 & 98.1 & 101.7 & 101.7 & 93.8\end{array}$

$\begin{array}{llllllllll}1989(9) & 92.7 & 101.4 & 102.3 & 103.0 & 98.6 & 97.7 & 101.9 & 101.1 & 94.3\end{array}$

$\begin{array}{llllllllll}1989(10) & 93.3 & 101.7 & 103.3 & 102.4 & 99.0 & 97.9 & 102.1 & 101.9 & 94.6\end{array}$

$\begin{array}{llllllllll}1989(11) & 93.6 & 102.4 & 102.4 & 99.1 & 99.1 & 99.1 & 102.4 & 102.4 & 95.2\end{array}$

$\begin{array}{llllllllll}1989(12) & 93.8 & 102.2 & 101.5 & 97.8 & 99.2 & 99.9 & 101.7 & 103.5 & 95.9\end{array}$

$\begin{array}{llllllllll}1990(1) & 96.9 & 103.4 & 99.4 & 100.8 & 100.2 & 101.1 & 102.6 & 104.5 & 96.8\end{array}$

$\begin{array}{llllllllll}1990(2) & 98.0 & 104.3 & 103.5 & 102.5 & 100.4 & 102.3 & 103.6 & 105.2 & 97.6\end{array}$

$\begin{array}{llllllllll}1990(3) & 97.9 & 104.5 & 106.9 & 103.9 & 100.8 & 104.1 & 103.6 & 105.2 & 98.4\end{array}$

$\begin{array}{llllllllll}1990(4) & 97.4 & 105.1 & 107.7 & 106.2 & 101.2 & 106.0 & 103.6 & 107.0 & 98.9\end{array}$ $\begin{array}{llllllllll}1983(3) & 71.5 & 81.1 & 89.5 & 105.9 & 83.7 & 72.0 & 93.4 & 79.7 & 62.5\end{array}$

$\begin{array}{llllllllll}1983(4) & 72.3 & 81.7 & 89.6 & 110.6 & 83.9 & 71.9 & 93.5 & 80.1 & 62.7\end{array}$

$\begin{array}{llllllllll}1983(5) & 73.2 & 81.9 & 90.6 & 116.6 & 85.4 & 73.0 & 93.6 & 80.2 & 63.0 \\ 1983(6) & 72.7 & 82.1 & 91.6 & 120.9 & 86.8 & 74.1 & 93.8 & 80.3 & 63.2\end{array}$

$\begin{array}{llllllllll}1983(7) & 72.3 & 82.3 & 91.1 & 122.4 & 87.5 & 75.6 & 94.0 & 80.2 & 63.7\end{array}$

$\begin{array}{llllllllll}1983(8) & 71.7 & 82.6 & 90.7 & 122.3 & 88.1 & 77.2 & 94.3 & 80.2 & 64.3\end{array}$

$\begin{array}{llllllllll}1983(9) & 72.0 & 82.5 & 91.0 & 120.0 & 88.1 & 77.2 & 94.6 & 80.4 & 64.3\end{array}$

$\begin{array}{llllllllll}1983(10) & 72.3 & 82.4 & 91.3 & 116.9 & 88.0 & 77.4 & 94.9 & 80.6 & 64.3\end{array}$

$\begin{array}{llllllllll}1983(11) & 72.4 & 82.2 & 91.3 & 114.6 & 88.2 & 77.6 & 94.9 & 81.1 & 64.5\end{array}$

$\begin{array}{llllllllll}1983(12) & 72.5 & 82.0 & 91.2 & 115.3 & 88.4 & 77.8 & 94.9 & 81.6 & 64.6\end{array}$

$\begin{array}{llllllllll}1984(1) & 74.4 & 82.4 & 90.4 & 114.8 & 88.4 & 77.3 & 94.8 & 81.7 & 65.1\end{array}$

$\begin{array}{llllllllll}1984(2) & 75.2 & 82.6 & 89.6 & 113.5 & 88.3 & 76.8 & 94.8 & 81.7 & 65.6\end{array}$

$\begin{array}{llllllllll}1984(3) & 75.3 & 82.7 & 90.2 & 112.6 & 89.1 & 76.5 & 95.0 & 81.5 & 65.9 \\ 1984(4) & 75.2 & 82.8 & 90.8 & 116.3 & 89.8 & 76.2 & 95.2 & 81.4 & 66.2\end{array}$

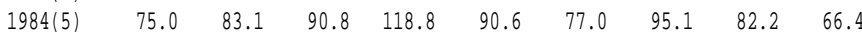

$\begin{array}{llllllllll}1984(6) & 74.9 & 83.4 & 90.8 & 118.7 & 91.3 & 77.8 & 95.1 & 82.8 & 66.5\end{array}$

$\begin{array}{llllllllll}1984(7) & 75.0 & 83.3 & 91.8 & 115.4 & 90.8 & 78.2 & 94.8 & 83.2 & 66.8\end{array}$

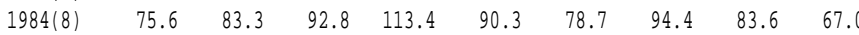

$\begin{array}{llllllllll}1984(9) & 75.6 & 83.7 & 93.3 & 114.6 & 90.9 & 78.8 & 94.4 & 84.0 & 67.2\end{array}$

$\begin{array}{llllllllll}1984(10) & 75.5 & 84.0 & 93.8 & 116.7 & 91.5 & 78.9 & 94.4 & 84.3 & 67.3\end{array}$

$\begin{array}{llllllllll}1984(11) & 75.7 & 83.9 & 93.8 & 117.2 & 91.8 & 78.7 & 94.4 & 84.5 & 67.6\end{array}$

$\begin{array}{llllllllll}1984(12) & 76.3 & 83.8 & 93.9 & 116.1 & 91.9 & 78.4 & 94.4 & 84.7 & 67.7\end{array}$

$\begin{array}{llllllllll}1985(1) & 76.9 & 84.2 & 93.7 & 112.4 & 91.5 & 78.5 & 95.0 & 85.0 & 68.2\end{array}$

$\begin{array}{llllllllll}1985(2) & 77.6 & 84.5 & 93.6 & 110.5 & 91.1 & 78.6 & 95.5 & 85.4 & 68.6\end{array}$

$\begin{array}{llllllllll}1985(3) & 77.2 & 84.8 & 94.6 & 114.4 & 92.3 & 79.4 & 95.9 & 85.5 & 69.2\end{array}$

$\begin{array}{llllllllll}1985(4) & 77.2 & 85.0 & 95.7 & 119.3 & 93.4 & 80.4 & 96.4 & 85.8 & 69.7\end{array}$

$\begin{array}{llllllllll}1985(5) & 77.0 & 85.1 & 95.6 & 121.6 & 93.6 & 80.4 & 96.0 & 86.3 & 70.1\end{array}$

$\begin{array}{llllllllll}1985(6) & 76.5 & 85.1 & 95.4 & 122.2 & 93.9 & 80.5 & 95.7 & 86.8 & 70.5\end{array}$

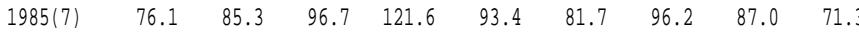

$\begin{array}{llllllllll}1985(8) & 76.3 & 85.4 & 98.2 & 118.2 & 93.0 & 82.9 & 96.7 & 87.1 & 72.0\end{array}$

$\begin{array}{llllllllll}1985(9) & 76.5 & 87.6 & 98.1 & 117.4 & 93.3 & 82.3 & 96.6 & 87.2 & 72.4\end{array}$

$\begin{array}{llllllllll}1985(10) & 76.4 & 89.8 & 98.1 & 117.1 & 93.4 & 81.7 & 96.5 & 87.3 & 72.6\end{array}$

$\begin{array}{llllllllll}1985(11) & 77.1 & 89.9 & 97.7 & 115.8 & 93.8 & 82.5 & 96.3 & 87.8 & 73.0\end{array}$

$\begin{array}{llllllllll}1985(12) & 77.9 & 90.0 & 97.4 & 115.6 & 94.1 & 83.2 & 96.0 & 88.3 & 73.4\end{array}$

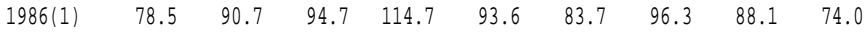

$\begin{array}{llllllllll}1986(2) & 78.0 & 91.3 & 92.1 & 109.7 & 93.2 & 84.3 & 96.6 & 87.8 & 74.6\end{array}$

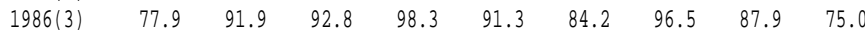

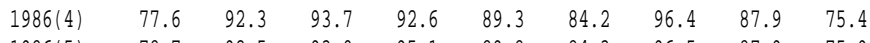

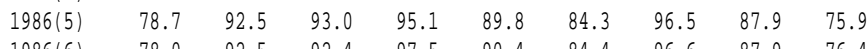

$\begin{array}{llllllllll}1986(6) & 78.0 & 92.5 & 92.4 & 97.5 & 90.4 & 84.4 & 96.6 & 87.9 & 76.4\end{array}$

$\begin{array}{llllllllll}1986(7) & 78.3 & 92.0 & 95.1 & 90.7 & 89.1 & 84.9 & 96.7 & 88.0 & 77.0\end{array}$

$\begin{array}{llllllllll}1986(8) & 78.7 & 91.5 & 97.8 & 84.7 & 87.8 & 85.6 & 96.7 & 88.2 & 77.6\end{array}$ $\begin{array}{llllllllll}1990(5) & 97.0 & 106.2 & 106.4 & 109.3 & 101.6 & 106.6 & 103.6 & 105.8 & 99.6\end{array}$ $\begin{array}{llllllllll}1990(6) & 97.2 & 107.0 & 104.9 & 113.1 & 102.5 & 107.1 & 104.0 & 106.6 & 99.9\end{array}$ $\begin{array}{llllllllll}1990(7) & 97.7 & 107.7 & 103.6 & 115.6 & 103.0 & 107.1 & 104.8 & 105.2 & 101.9\end{array}$ $\begin{array}{llllllllll}1990(8) & 97.7 & 106.8 & 104.7 & 125.0 & 104.6 & 107.2 & 104.3 & 105.8 & 102.6\end{array}$ $\begin{array}{llllllllll}1990(9) & 98.0 & 107.5 & 107.8 & 133.6 & 106.3 & 109.7 & 105.0 & 106.0 & 103.1\end{array}$ $\begin{array}{llllllllll}1990(10) & 98.6 & 107.6 & 108.8 & 140.4 & 108.2 & 112.0 & 105.4 & 106.4 & 104.1\end{array}$ $\begin{array}{llllllllll}1990(11) & 99.6 & 107.2 & 107.6 & 140.8 & 108.8 & 114.1 & 103.6 & 107.0 & 104.7\end{array}$ $\begin{array}{llllllllll}1990(12) & 99.5 & 107.1 & 106.5 & 139.5 & 108.9 & 117.0 & 103.7 & 107.3 & 105.1\end{array}$ $\begin{array}{llllllllll}1991(1) & 102.9 & 113.4 & 106.2 & 127.7 & 106.6 & 117.1 & 104.3 & 108.1 & 106.4\end{array}$ $\begin{array}{llllllllll}1991(2) & 101.9 & 116.1 & 107.0 & 116.3 & 104.6 & 118.3 & 104.9 & 109.4 & 107.3\end{array}$ $\begin{array}{llllllllll}1991(3) & 101.9 & 117.2 & 108.3 & 106.4 & 102.6 & 115.7 & 105.0 & 109.1 & 108.0\end{array}$ $\begin{array}{llllllllll}1991(4) & 103.0 & 117.0 & 110.2 & 107.4 & 102.6 & 109.9 & 105.9 & 110.2 & 108.2\end{array}$ $\begin{array}{llllllllll}1991(5) & 102.6 & 117.4 & 108.3 & 114.3 & 104.2 & 108.9 & 105.7 & 110.8 & 108.9\end{array}$ $\begin{array}{llllllllll}1991(6) & 102.9 & 117.3 & 105.5 & 116.3 & 105.1 & 109.4 & 105.4 & 110.9 & 109.6\end{array}$ $\begin{array}{llllllllll}1991(7) & 101.3 & 118.5 & 106.6 & 112.9 & 105.1 & 110.2 & 105.5 & 111.3 & 110.8\end{array}$ $\begin{array}{llllllllll}1991(8) & 100.0 & 119.3 & 108.9 & 114.7 & 105.7 & 111.2 & 106.3 & 111.9 & 111.3\end{array}$ $\begin{array}{llllllllll}1991(9) & 99.8 & 119.8 & 110.5 & 118.1 & 106.1 & 108.2 & 106.8 & 111.2 & 111.9\end{array}$ $\begin{array}{llllllllll}1991(10) & 100.0 & 120.2 & 110.8 & 116.0 & 106.0 & 106.5 & 105.6 & 112.1 & 112.4\end{array}$ $\begin{array}{llllllllll}1991(11) & 101.5 & 119.8 & 112.3 & 116.7 & 106.4 & 108.7 & 105.1 & 111.9 & 113.1\end{array}$ $\begin{array}{llllllllll}1991(12) & 102.1 & 119.5 & 109.7 & 114.9 & 106.5 & 111.8 & 104.5 & 111.3 & 113.6\end{array}$ $\begin{array}{llllllllll}1992(1) & 102.5 & 121.1 & 109.0 & 111.8 & 106.5 & 111.7 & 105.8 & 113.5 & 114.5\end{array}$ $\begin{array}{llllllllll}1992(2) & 102.7 & 121.0 & 110.8 & 112.7 & 106.7 & 110.0 & 106.8 & 114.0 & 115.6\end{array}$ $\begin{array}{llllllllll}1992(3) & 103.9 & 121.5 & 113.3 & 113.7 & 106.9 & 111.7 & 107.2 & 113.8 & 116.2\end{array}$ $\begin{array}{llllllllll}1992(4) & 103.5 & 122.3 & 113.3 & 116.7 & 107.6 & 111.2 & 107.7 & 114.7 & 117.0\end{array}$ $\begin{array}{llllllllll}1992(5) & 102.2 & 122.7 & 114.4 & 124.3 & 109.0 & 110.8 & 106.7 & 114.0 & 117.4\end{array}$ $\begin{array}{llllllllll}1992(6) & 102.2 & 122.9 & 111.4 & 129.4 & 110.4 & 105.7 & 107.4 & 114.3 & 118.0\end{array}$ $\begin{array}{llllllllll}1992(7) & 101.5 & 122.9 & 109.4 & 130.9 & 110.8 & 108.7 & 108.4 & 116.2 & 119.2\end{array}$ $\begin{array}{llllllllll}1992(8) & 102.8 & 122.5 & 110.6 & 130.2 & 110.8 & 106.5 & 107.9 & 117.2 & 119.7\end{array}$ $\begin{array}{llllllllll}1992(9) & 103.6 & 122.6 & 111.3 & 128.8 & 110.5 & 107.6 & 108.2 & 116.0 & 120.4\end{array}$ $\begin{array}{llllllllll}1992(10) & 103.7 & 123.1 & 112.7 & 128.6 & 111.2 & 111.8 & 108.1 & 116.6 & 121.2\end{array}$ $\begin{array}{llllllllll}1992(11) & 103.2 & 122.1 & 113.0 & 130.7 & 112.0 & 112.7 & 108.4 & 117.6 & 121.4\end{array}$ $\begin{array}{llllllllll}1992(12) & 103.8 & 122.2 & 110.5 & 128.4 & 111.4 & 115.5 & 108.1 & 116.9 & 121.9\end{array}$

Source: The ACCRA data for Provo, Utah and Salt Lake City, Utah were used to convert CPI data for the cities of Provo and Salt Lake City in the West Region of the United States. These Provo and Salt Lake City price indices were then used to construct a price index for Utah, relative to a US average price for each of the goods, in November, 1989, listed above. 